Distribution Category:

Magnetic Fusion Energy Systems

(UC-424)

\author{
ARGONNE NATIONAL LABORATORY \\ 9700 South Cass Avenue \\ Argonne, Illinois 60439-4801
}

\title{
SUMMARY REPORT FOR ITER TASK - D4: ACTIVATION CALCULATIONS FOR THE LITHIUM VANADIUM ITER DESIGN
}

\author{
by \\ Hosny Attaya \\ Fusion Power Program/Technology Development Division
}

February 1995

DISTRIBUTION OF THIS DOCUMENT IS UNLIMITED

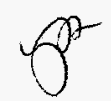

Work supported by the

Office of Fusion Energy

U.S. Department of Energy

under Contract W-31-109-Eng-38 


\section{DISCLAIMER}

This report was prepared as an account of work sponsored by an agency of the United States Government. Neither the United States Government nor any agency thereof, nor any of their employees, make any warranty, express or implied, or assumes any legal liability or responsibility for the accuracy, completeness, or usefulness of any information, apparatus, product, or process disclosed, or represents that its use would not infringe privately owned rights. Reference herein to any specific commercial product, process, or service by trade name, trademark, manufacturer, or otherwise does not necessarily constitute or imply its endorsement, recommendation, or favoring by the United States Government or any agency thereof. The views and opinions of authors expressed herein do not necessarily state or reflect those of the United States Government or any agency thereof. 


\section{DISCLAIMER}

Portions of this document may be illegible in electronic image products. Images are produced from the best available original document. 


\section{TABLE OF CONTENTS}

$\underline{\text { Page }}$

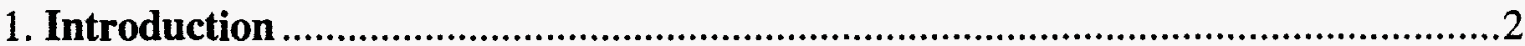

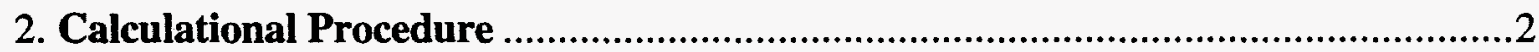

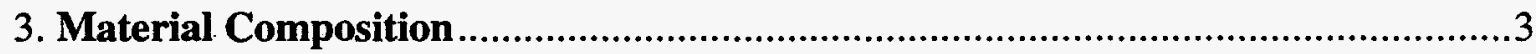

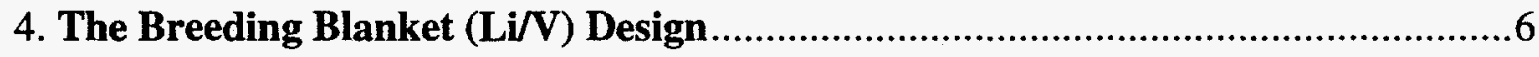

4.1 Radial Build and Neutron Flux......................................................................

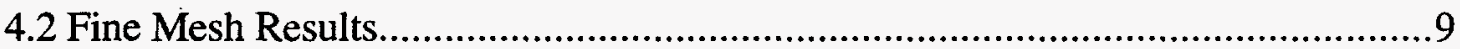

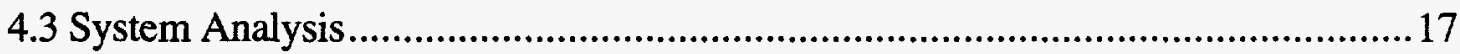

4.4 Contact Dose

4.5 Detailed Zones' Results............................................................................27;

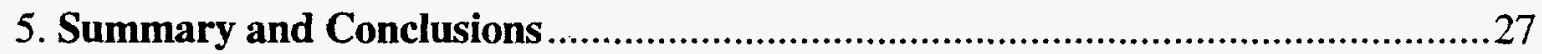

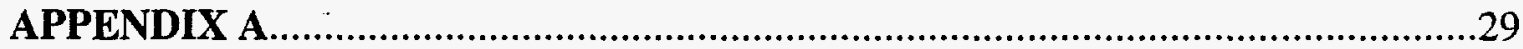

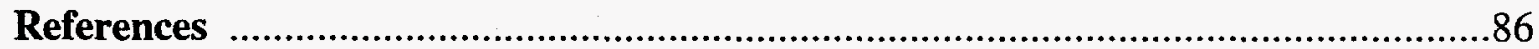




\section{LIST OF FIGURES}

Page

Figure 1. The midplane radial build of ITER, the neutron, and the photons fluxes $(\mathrm{Li} / \mathrm{V}$ design).

Figure 2. Isometric view of the inboard neutron flux (logarithmic scale) as a function of the lethargy and radius ( $\mathrm{Li} / \mathrm{V}$ design).

Figure 3. Isometric view of the outboard neutron flux (logarithmic scale) as a function of the lethargy and radius ( $\mathrm{Li} / \mathrm{V}$ design)...

Figure 4. The specific radioactivity distribution at different times after shutdown ( $\mathrm{Li} / \mathrm{V}$ design).

Figure 5. The specific decay heat distribution at different times after shutdown $(\mathrm{Li} / \mathrm{V}$ design).

Figure 6. The specific air-BHP distribution at different times after shutdown $(\mathrm{Li} / \mathrm{V}$ design).

Figure 7. The point-source contact dose distribution at different times after shutdown ( Li/ $\mathrm{V}$ design).

Figure 8 . The total radioactivity (per $\mathrm{cm}$ height) in the system, top: isotopic contributions, bottom: zonal contributions ( $\mathrm{Li} / \mathrm{V}$ design).

Figure 9. The total decay heat (per cm height) in the system, top: isotopic contributions, bottom: zonal contributions ( $\mathrm{Li} / \mathrm{V}$ design).

Figure 10. The total air BHP (per $\mathrm{cm}$ height) in the system, top: isotopic contributions, bottom: zonal contributions ( $\mathrm{Li} / \mathrm{V}$ design).

Figure 11. The contact dose (with photon transport) at different times after shutdown and after 3.29 FPYs ( $\left.3 \mathrm{MWa} / \mathrm{m}^{2}-\mathrm{Li} / \mathrm{V}\right)$.

Figure 12. The contact dose, without the VV's INCONEL photon source, at different times after shutdown and after 3.29 FPYs $\left(3 \mathrm{MWa} / \mathrm{m}^{2}-\mathrm{Li} / \mathrm{V}\right)$

Figure 13. The contact dose, with only the vanadium photon source, at different times after shutdown and after 3.29 FPYs $\left(3 \mathrm{MWa} / \mathrm{m}^{2}-\mathrm{Li} / \mathrm{V}\right)$.

Figure 14. The contact dose (with photon transport) at different times after shutdown and after $0.11 \mathrm{FPYs}\left(0.1 \mathrm{MWa} / \mathrm{m}^{2}-\mathrm{Li} / \mathrm{V}\right)$.

Figure 15. The specific radioactivity (top) and the specific decay heat (bottom) in zone \# 16 (I_Vall - Li/V).

Figure 16. The specific air-BHP (top) and the point-source contact dose (bottom) in zone \# 16 (I_Vall - Li/V).

Figure 17. The specific radioactivity (top) and the specific decay heat (bottom) in zone \# $15\left(\mathrm{I} \_\mathrm{Li}-\mathrm{Li} / \mathrm{V}\right)$. 
Figure 18. The specific air-BHP in zone \# 15 (I_Li - Li/V)

Figure 19. The specific radioactivity (top) and the specific decay heat (bottom) in zone \# 14 (I_BeZ - Li/V).

Figure 20. The specific air-BHP (top) and the point-source contact dose (bottom) in zone \# 14 (I_BeZ - Li/V).

Figure 21. The specific radioactivity (top) and the specific decay heat (bottom) in zone \# $13\left(\mathrm{I} \_\mathrm{Li}-\mathrm{Li} / \mathrm{V}\right)$. .36

Figure 22. The specific air-BHP in zone \# $13\left(\mathrm{I} \_\mathrm{Li}-\mathrm{Li} / \mathrm{V}\right)$. .37

Figure 23. The specific radioactivity (top) and the specific decay heat (bottom) in zone \# 12 (I_Vall - Li/V).

Figure 24. The specific air-BHP (top) and the point-source contact dose (bottom) in zone \# 12 (I_Vall - Li/V).

Figure 25. The specific radioactivity (top) and the specific decay heat (bottom) in zone \# $11\left(\mathrm{I} \_\mathrm{Li}-\mathrm{Li} / \mathrm{V}\right)$.

Figure 26. The specific air-BHP in zone \# $11\left(\mathrm{I} \_\mathrm{Li}-\mathrm{Li} / \mathrm{V}\right)$.

Figure 27. The specific radioactivity (top) and the specific decay heat (bottom) in zone \# $10($ I_WC - Li/V).

Figure 28. The specific air-BHP (top) and the point-source contact dose (bottom) in zone \# 10 (I_WC - Li/V).

Figure 29. The specific radioactivity (top) and the specific decay heat (bottom) in zone \# 9 (I_Li - Li/V).

Figure 30. The specific air-BHP in zone \# 9 (I_Li - Li/V).

Figure 31. The specific radioactivity (top) and the specific decay heat (bottom) in zone \# 8 (I_Vall - Li/V)

Figure 32. The specific air-BHP (top) and the point-source contact dose (bottom) in zone \# 8 (I_Vall - Li/V).

Figure 33. The specific radioactivity (top) and the specific decay heat (bottom) in zone \# 7 (I_Incon - Li/V).

Figure 34. The specific air-BHP (top) and the point-source contact dose (bottom) in zone \# 7 (I_Incon - Li/V).

Figure 35. The specific radioactivity (top) and the specific decay heat (bottom) in zone \# 6 (I_WNaK - Li/V).

Figure 36. The specific air-BHP (top) and the point-source contact dose (bottom) in zone \# 6 (I_WNaK - Li/V).

Figure 37. The specific radioactivity (top) and the specific decay heat (bottom) in zone \# 5 (I_Incon - Li/V). 
Figure 38. The specific air-BHP (top) and the point-source contact dose (bottom) in zone \# 5 (I_Incon - Li/V).

Figure 39. The specific radioactivity (top) and the specific decay heat (bottom) in zone \# 4 (I_B4C_Pb - Li/V).

Figure 40. The specific air-BHP (top) and the point-source contact dose (bottom) in zone \# 4 (I_B4C_Pb - Li/V).

Figure 41. The specific radioactivity (top) and the specific decay heat (bottom) in zone \# 3 (I_SSWP - Li/V).

Figure 42. The specific air-BHP (top) and the point-source contact dose (bottom) in zone \# 3 (I_SSWP - Li/V).

Figure 43. The specific radioactivity (top) and the specific decay heat (bottom) in zone \# 2 (I_coil - Li/V).

Figure 44. The specific air-BHP (top) and the point-source contact dose (bottom) in zone \# 2 (I_coil - Li/V).

Figure 45. The specific radioactivity (top) and the specific decay heat (bottom) in zone \# 1 (I_SSWP - Li/V).

Figure 46. The specific air-BHP (top) and the point-source contact dose (bottom) in zone \# 1 (I_SSWP - Li/V).

Figure 47. The specific radioactivity (top) and the specific decay heat (bottom) in zone \# 17 (O_Vall - Li/V).

Figure 48. The specific air-BHP (top) and the point-source contact dose (bottom) in zone \# 17 (O_Vall - Li/V).

Figure 49. The specific radioactivity (top) and the specific decay heat (bottom) in zone \# $18\left(\mathrm{O} \_\mathrm{Li}\right.$ - Li/V).

Figure 50. The specific air-BHP in zone \#18 $\left(\mathrm{O}_{-} \mathrm{Li}-\mathrm{Li} / \mathrm{V}\right)$.

Figure 51. The specific radioactivity (top) and the specific decay heat (bottom) in zone \# 19 (O_BeZ - Li/V).

Figure 52. The specific air-BHP (top) and the point-source contact dose (bottom) in zone \# 19 (O_BeZ - Li/V).

Figure 53. The specific radioactivity (top) and the specific decay heat (bottom) in zone \# $20\left(\mathrm{O} \_\mathrm{Li}\right.$ - Li/V). .68

Figure 54. The specific air-BHP in zone \# $20\left(\mathrm{O} \_\mathrm{Li}-\mathrm{Li} / \mathrm{V}\right)$.

Figure 55. The specific radioactivity (top) and the specific decay heat (bottom) in zone \# $21\left(O_{-}\right.$Vall - Li/V).

Figure 56. The specific air-BHP (top) and the point-source contact dose (bottom) in zone \# 21 (O_Vall - Li/V). 
Figure 57. The specific radioactivity (top) and the specific decay heat (bottom) in zone \# $22\left(\mathrm{O} \_\mathrm{Li}-\mathrm{Li} / \mathrm{V}\right)$.

Figure 58. The specific air-BHP in zone \# $22\left(\mathrm{O} \_\mathrm{Li}-\mathrm{Li} / \mathrm{V}\right)$.

Figure 59. The specific radioactivity (top) and the specific decay heat (bottom) in zone \# 23 (O_WC - Li/V). 74

Figure 60. The specific air-BHP (top) and the point-source contact dose (bottom) in zone \# 23 (O_WC - Li/V)

Figure 61. The specific radioactivity (top) and the specific decay heat (bottom) in zone \# $24\left(\mathrm{O} \_\mathrm{Li}-\mathrm{Li} / \mathrm{V}\right)$. .76

Figure 62. The specific air-BHP in zone \# $24\left(\mathrm{O} \_\mathrm{Li}-\mathrm{Li} / \mathrm{V}\right)$. .77

Figure 63. The specific radioactivity (top) and the specific decay heat (bottom) in zone \# $25\left(\mathrm{O}_{-}\right.$Vall - Li/V).

Figure 64. The specific air-BHP (top) and the point-source contact dose (bottom) in zone \# 25 (O_Vall - Li/V).

Figure 65. The specific radioactivity (top) and the specific decay heat (bottom) in zone \# $26($ O_Incon - $\mathrm{Li} / \mathrm{V})$.

Figure 66. The specific air-BHP (top) and the point-source contact dose (bottom) in zone \# 26 (O_Incon - Li/V).

Figure 67. The specific radioactivity (top) and the specific decay heat (bottom) in zone \# 27 (O_WNaK - Li/V).

Figure 68. The specific air-BHP (top) and the point-source contact dose (bottom) in zone \# 27 (O_WNaK - Li/V).

Figure 69. The specific radioactivity (top) and the specific decay heat (bottom) in zone \# $28\left(O_{-}\right.$Incon - Li $\left./ \mathrm{V}\right)$.

Figure 70. The specific air-BHP (top) and the point-source contact dose (bottom) in zone \# 28 (O_Incon - Li/V). 


\section{LIST OF TABLES}

$\underline{\text { Page }}$

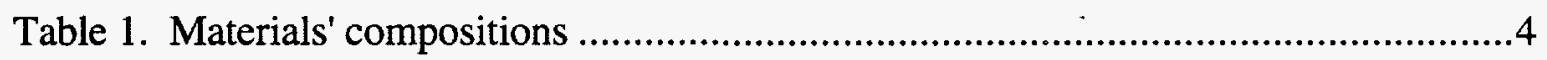

Table 2. Zonal volume compositions (\%), ...................................................................

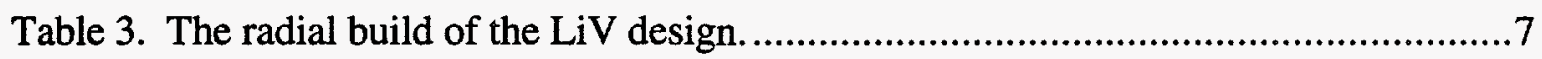

Table 4. The materials' masses used in all the zones...................................................... 


\section{Summary Report for ITER Task - D4: \\ Activation Calculations for the Lithium Vanadium ITER Design}

Hosny Attaya

\section{EXECUTIVE SUMMARY}

Detailed activation analysis for ITER has been performed as a part of ITER Task D4. The calculations have been performed for the shielding blanket (SS/water) and for the breeding blanket $(\mathrm{Li} / \mathrm{V})$ options. The activation code RACC-P, which has been modified under ITER Task-D-10 for pulsed operation, has been used in this analysis. The spatial distributions of the radioactive inventory, decay heat, biological hazard potential, and the contact dose were calculated for the two designs for different operation modes and targeted fluences.

A one-dimensional toroidal geometrical model has been utilized to determine the neutron fluxes in the two designs. The results are normalized for an inboard and outboard neutron wall loadings of 0.91 and $1.2 \mathrm{MW} / \mathrm{m}^{2}$, respectively.

The point-wise distributions of the decay gamma sources have been calculated everywhere in the reactor at several times after the shutdown of the two designs and are then used in the transport code ONEDANT to calculate the biological dose everywhere in the reactor. The point-wise distributions of all the responses have also been calculated. These calculations have been performed for neutron fluences of $3.0 \mathrm{MWa} / \mathrm{m}^{2}$, which corresponds to the target fluence of ITER, and $0.1 \mathrm{MWa} / \mathrm{m}^{2}$, which is anticipated to correspond to the beginning of the extended maintenance period.

The decay heat results show that a large fraction of this energy (50 to 90\%) is produced by photons. This implies that this energy would be transported to different parts of the reactor, thus relieving the energy concentration at high intensity source locations such as the first wall. Accurate modeling for the decay gamma transport is required to produce realistic spatial distribution of the decay heat which may be used in LOCA and LOFA analyses.

The results of the pulsed operation, using the new version of RACC, show large reductions in the radioactivity and the decay heat for pure pulsed operation. The continuous operation assumption, usually used in the radioactivity calculations, overestimates the different activation responses at the final shutdown and for about one week after shutdown by a factor of more than 2 . 


\section{Introduction}

Part of this design task is to perform activation calculations for the different parts of ITER to provide information needed for the engineering design, maintenance assessment, safety analysis, and radwaste management. For this purpose, detailed activation analyses have been carried out for the two ITER designs, the SS/water design and the LiV (breeding) design. For both designs, the radial build, the neutron wall loading, and the operation scenarios, used in these calculations, were provided by the JCT.

The activation code RACC-P [1], which has been modified under ITER Task-D10 for pulsed operation [2], has been used in this analysis. The spatial distributions of the radioactive inventory, decay heat, biological hazard potential, and the contact dose were calculated for the two designs for different operation modes and targeted fluences. In this report the activation results of the lithium vanadium breeding design $(\mathrm{Li} / \mathrm{V})$ are presented.

\section{Calculational Procedure}

There is no reliable multidimensional deterministic transport computer code that is capable of adequately modeling the complicated tokamak geometry and calculating the neutron flux, which is the key input for the activation calculations, accurately. On the other hand, utilizing the neutron flux calculated by 3-D Monte Carlo methods in the activation calculations would impregnate its results with the large statistical errors inherent to the calculated Monte Carlo flux. Thus, for the activation calculations, a onedimensional cylindrical toroidal geometrical model has been used in the neutron transport calculations. In this model, the cylinder axis is the vertical axis of the reactor, and the inboard and the outboard blankets/shields are modeled with their midplane compositions. The neutron source is extended between the midplane plasma boundaries. This allows for the mutual neutronics coupling between the inboard and the outboard blankets/shields as well as the geometrical toroidal effect on the calculated neutron flux. 
It is important to realize the limitations of this modeling and to understand the employed approximations in order to interpret the obtained activation results correctly. The first obvious assumption in this model is the full coverage of the FW/blanket/shield, i.e., no account is made for the large penetrations in the reactor. The second assumption is the discount of any poloidal variations of the neutron wall loading, the neutron source incident angle, and the radial build of the blanket/shield. The model deals with and produces average vertical (poloidal) values representing part of the system rather than localized values that could deviate considerably from the system average. Another assumption is the nucleonics decoupling of that part of the system, modeled in the calculations, from the rest of the system. The larger the modeled part is, the less effect this assumption has. These assumptions, in general, tend to overestimate the radioactivity.

\section{Material Composition}

The weight and the atomic compositions of the different materials used in these calculations are given in Table 1. In this table, the mass density $(\mathrm{gm} / \mathrm{cc})$ and the atomic density (atoms-barn/cc) are listed first for each material, then the mass and atomic densities of each element are given in percentage or in ppm (marked by *).

Table 2 shows the volume compositions of the different mixtures made up from the basic materials (Table 1), and assigned to different geometrical zones. The names and acronyms used in these two tables are keys to understanding all of the graphics outputs. 
Table 1. Materials' compositions

\begin{tabular}{|c|c|c|c|c|c|}
\hline Alloy: & SS316-L & Inci625 & SS316lw & $\mathrm{v} 4 \mathrm{cr} 4 \mathrm{ti}$ & repxy \\
\hline & $7.855: 8.528-2$ & $8.44 \quad: 8.632-2$ & $7.855: 8.543-2$ & $6.1 \quad: 7.235-2$ & $1.9006: 8.421-2$ \\
\hline & : & : & 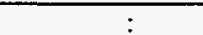 & : & : \\
\hline $\mathrm{H}$ & $\therefore \ldots$ & ...................... & $\therefore \ldots \ldots$ & …................ & $1.90: 25.69$ \\
\hline B & $* 10.00: * 51.30$ & ........................ & $* 10.00: * 51.21$ & ....................... & .................... \\
\hline C & $0.02: 0.08$ & $0.10: 0.49$ & $0.01: 0.05$ & $* 50.00: 0.02$ & $19.85: 22.47$ \\
\hline $\mathbf{N}$ & $0.07: 0.28$ & 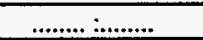 & $0.19: 0.75$ & $0.01: 0.04$ & $2.52: 2.45$ \\
\hline 0 & $* 20.00: * 69.33$ & $\ldots \ldots \ldots \ldots$ & $* 20.00: * 69.21$ & $0.03: 0.10$ & $37.82: 32.13$ \\
\hline $\mathrm{Mg}$ & ..................... & .................... & ........................ & ...................... & $2.53: 1.41$ \\
\hline $\mathrm{Al}$ & $0.30: 0.62$ & $0.40: 0.87$ & $0.30: 0.62$ & $0.02: 0.04$ & $9.26: 4.67$ \\
\hline $\mathrm{Si}$ & $0.46: 0.91$ & $0.50: 1.05$ & $0.10: 0.20$ & $0.04: 0.07$ & $19.63: 9.50$ \\
\hline $\mathrm{P}$ & $0.03: 0.05$ & $0.02: 0.04$ & $* 30.00: * 53.63$ & $* 4.00: * 6.56$ & $\therefore . . .$. \\
\hline $\mathrm{S}$ & $0.01: 0.02$ & $0.02: 0.04$ & $* 14.00: * 24.17$ & $* 3.00: * 4.75$ & $1.43: 0.61$ \\
\hline $\mathrm{Cl}$ & .................... & 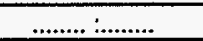 & .................... & $* 1.00: * 1.43$ & $\ldots \ldots \ldots . . . . . . . . .$. \\
\hline $\mathrm{K}$ & $* 5.00: * 7.09$ & ……............. & $* 5.00: * 7.08$ & ${ }^{*} 0.10: * 0.13$ & $\ldots \ldots \ldots$ \\
\hline $\mathrm{Ti}$ & $0.04: 0.05$ & $0.40: 0.49$ & $0.04: 0.05$ & $4.00: 4.24$ & $\ldots \ldots$ \\
\hline $\mathrm{V}$ & $* 40.00: * 43.55$ & ....................... & $* 40.00: * 43.48$ & $91.89: 91.58$ & 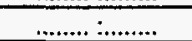 \\
\hline $\mathrm{Cr}$ & $17.10: 18.24$ & $22.00: 24.91$ & $17.17: 18.28$ & $4.00: 3.91$ & ……: :........... \\
\hline Mn & $1.70: 1.72$ & $0.50: 0.54$ & $1.48: 1.49$ & $\ldots \ldots \ldots . \ldots \ldots$ & $\ldots \ldots . . . \ldots \ldots . . . .$. \\
\hline $\mathrm{Fe}$ & $64.43: 63.99$ & $5.00: 5.27$ & $66.33: 65.77$ & $* 40.00: * 36.37$ & ....................... \\
\hline $\mathrm{Co}$ & $0.03: 0.03$ & $1.00: 1.00$ & $0.03: 0.03$ & ........................ & …................. \\
\hline $\mathrm{Ni}$ & $13.20: 12.47$ & $57.05: 57.24$ & $12.14: 11.45$ & $* 3.00: * 2.60$ & $\ldots \ldots \ldots \ldots$ \\
\hline $\mathrm{Cu}$ & $0.10: 0.09$ & $\ldots \ldots \ldots$ :.......... & $0.10: 0.09$ & $* 2.00: * 1.60$ & $5.05: 1.08$ \\
\hline As & $* 5.00: * 3.70$ & ................... & $* 5.00: * 3.70$ & ${ }^{*} 2.00: * 1.36$ & 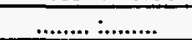 \\
\hline $\mathrm{Zr}$ & $* 20.00: * 12.16$ & 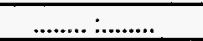 & $* 20.00: * 12.14$ & $* 1.00: * 0.56$ & ....................... \\
\hline $\mathrm{Nb}$ & $* 20.00: * 11.94$ & $4.00: 2.54$ & $0.05: 0.03$ & $* 0.50: * 0.27$ & .................... \\
\hline Mo & $2.50: 1.45$ & $9.00: 5.52$ & $2.03: 1.17$ & $* 4.00:^{*} 2.12$ & …............... \\
\hline $\mathrm{Ag}$ & $* 2.00: * 1.03$ & …................. & $* 2.00: * 1.03$ & $* 0.01: * 0.00$ & …................ \\
\hline Cd & $* 2.00: * 0.99$ & ……............. & $* 2.00: * 0.99$ & 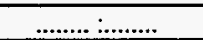 & …................ \\
\hline Sn & $* 20.00: * 9.34$ & & $* 20.00:^{*} 9.33$ & …............. & ................... \\
\hline $\mathrm{Sb}$ & * $5.00:{ }^{*} 2.28$ & & $* 5.00: * 2.27$ & & $\ldots \ldots \ldots \ldots \ldots$ \\
\hline $\mathrm{Ba}$ & $* 5.00: * 2.02$ & .................... & $* 5.00: * 2.02$ & $\ldots \ldots \ldots$............ & …................. \\
\hline $\mathrm{Tb}$ & $* 5.00: * 1.75$ & …............... & $* 5.00: * 1.74$ & …................ & $\ldots \ldots \ldots . \ldots \ldots$ \\
\hline $\mathrm{Ta}$ & $* 5.00: * 1.53$ & $* 50.00: * 16.27$ & $* 5.00: * 1.53$ & $* 0.20: * 0.06$ & 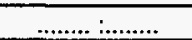 \\
\hline W & $* 10.00: * 3.02$ & $\ldots \ldots \ldots \ldots$ & $* 10.00: * 3.01$ & $* 1.00: * 0.28$ & $\ldots \ldots: \ldots \ldots \ldots$ \\
\hline Ir & $* 5.00: * 1.44$ & $\ldots \ldots \ldots . . . \ldots \ldots$ & $* 5.00: * 1.44$ & $\ldots \ldots . . . . . . . . . . .$. & ........................ \\
\hline $\mathrm{Pb}$ & $* 8.00: * 2.14$ & $\ldots \ldots \ldots$ & $* 8.00: * 2.14$ & $\ldots \ldots \ldots \ldots \ldots \ldots$ & $\therefore \ldots \ldots . . . .$. \\
\hline $\mathrm{Bi}$ & $* 8.00: * 2.12$ & 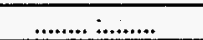 & $* 8.00: * 2.12$ & …............... & \\
\hline
\end{tabular}

\begin{tabular}{|c|c|c|c|c|c|}
\hline & nb3sn & $b 4 c$ & cube & L_nak68 & wc \\
\hline & $.5 .455-2$ & $2.52: 1.373-1$ & $8.87461: 8.679-2$ & $0.81691: 1.748-2$ & $15.62606: 9.614-2$ \\
\hline & $:$ & $:$ & : & $:$ & $:$ \\
\hline $\mathrm{Be}$ & ….................... & 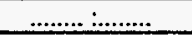 & $0.50: 3.42$ & ……................ & $\ldots . . . . . . . . . . . . .$. \\
\hline B & $\ldots \ldots \ldots . . . \ldots \ldots$ & $78.26: 80.00$ & $\ldots \ldots \ldots \ldots$ & 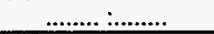 & $\therefore \ldots . . . .$. \\
\hline $\mathrm{C}$ & …................ & $21.74: 20.00$ & …................ & ..................... & $6.14: 50.03$ \\
\hline $\mathrm{Na}$ & ................... & .................... & ........................ & $55.55: 68.00$ & …................ \\
\hline $\mathrm{K}$ & $\ldots \ldots \ldots . . . . \ldots . . . .$. & $\ldots \ldots \ldots . \ldots \ldots . . . . . .$. & …................. & $44.45: 32.00$ & $\ldots \ldots \ldots . . . . . \ldots \ldots$ \\
\hline $\mathrm{Ni}$ & ㄷ……….... & $\ldots \ldots \ldots: \ldots \ldots$ & $2.00: 2.10$ & …................. & $\ldots \ldots \ldots \ldots$ \\
\hline $\mathrm{Cu}$ & 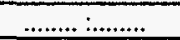 & 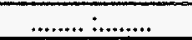 & $97.50: 94.48$ & $\ldots \ldots \ldots \ldots \ldots$ & $\ldots \ldots \ldots \ldots \ldots$ \\
\hline $\mathrm{Nb}$ & $70.13: 75.00$ & .................... & …................. & ................... & $\ldots \ldots \ldots \ldots$ \\
\hline Sn & $29.87: 25.00$ & …................ & 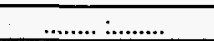 & …................. & $\ldots \ldots: \ldots \ldots \ldots$ \\
\hline W & $0^{\circ}$ & $\cdot$ & $\ldots \ldots \ldots \ldots$ & $\ldots \ldots \ldots+\ldots \ldots$ & $93.86: 49.97$ \\
\hline
\end{tabular}


Table 2. Zonal volume compositions (\%).

\begin{tabular}{|c|c|c|c|c|c|c|c|c|c|}
\hline Mix: & SSWP & coil & B4C_Pb & Incon & WNaK & Cua & SS_H20 & SS & $\mathrm{H} 2 \mathrm{O}$ \\
\hline material & $\%$ & $\%$ & $\%$ & $\%$ & $\%$ & $\%$ & $\%$ & $\%$ & $\%$ \\
\hline ss $316-L$ & .......... & ........... & $\ldots$. & $\ldots \ldots . .$. & $\ldots \ldots \ldots$ & ........... & 60. & 100. & $\ldots . .$. \\
\hline incl625 & $\ldots \ldots \ldots$ & $\ldots \ldots \ldots$ & $\ldots$ & 100. & ......... & ........... & ......... & ......... & $\ldots \ldots$ \\
\hline ss316lw & 100. & 47. & $\ldots$ & .......... & ........... & ........... & ........... & .......... & $\ldots .$. \\
\hline$r-e p x y$ & ......... & 13.3 & $\ldots \ldots . . .$. & .......... & ................ & .......... & ............. & ........... & $\ldots \ldots$ \\
\hline nb3sn & $\ldots \ldots \ldots$ & 10.5 & ........... & ........... & ............. & .......... & $\ldots \ldots \ldots$ & .......... & $\ldots \ldots$ \\
\hline$b 4 c$ & .......... & .......... & 40. & $\ldots . . . . .$. & ......... & $\ldots \ldots \ldots$ & $\ldots \ldots \ldots$ & $\ldots \ldots \ldots$ & ....... \\
\hline cube & …....... & ............ & $\ldots$ & ........... & …...... & 100. & …........ & .............. & $\ldots . .$. \\
\hline L_nak68 & ......... & ........ & ............ & ........... & 20. & ......... & ......... & .......... & ..... \\
\hline WC & ........... & ........... & ............ & ........ & 80. & $\ldots \ldots \ldots$ & $\ldots \ldots \ldots$ & $\ldots \ldots$. & .... \\
\hline h2o & ............ & .......... & $\ldots \ldots \ldots$ & ........... & …........ & ........... & 40. & ......... & 100. \\
\hline $\mathrm{cu} \_$ITR & ........... & 12. & $\ldots \ldots \ldots$ & & $\ldots \ldots \ldots$ & .......... & $\ldots \ldots \ldots$ & & \\
\hline pb_p & $\ldots \ldots$ & $\ldots \ldots \ldots$ & 60. & $\ldots \ldots \ldots$ & $\ldots . . . .$. & ........... & $\ldots \ldots \ldots$ & .......... & 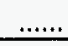 \\
\hline lhe & $\ldots \ldots \ldots$ & 17.2 & ........ & $\ldots \ldots \ldots$ & $\ldots \ldots \ldots$ & $\ldots \ldots \ldots$ & $\ldots \ldots \ldots$ & $\ldots \ldots \ldots$ & \\
\hline
\end{tabular}

\begin{tabular}{|c|c|c|c|c|}
\hline Mix: & Vall & $\mathrm{Li}$ & WC & $\mathrm{BeZ}$ \\
\hline Material & $\%$ & $\%$ & $\%$ & $\%$ \\
\hline $\mathrm{v} 4 \mathrm{cr} 4 \mathrm{ti}$ & 100. & $\ldots$ & $\ldots \ldots \ldots$ & $\ldots$ \\
\hline wc & ......... & ......... & 100. & $\ldots$ \\
\hline L_li & ......... & 100. & $\ldots \ldots .$. & ......... \\
\hline be 100 & ......... & .......... & ......... & 100. \\
\hline
\end{tabular}




\section{The Breeding Blanket (Li/V) Design}

This is an alternative design option for ITER that is aimed at tritium selfsufficiency, reactor relevancy, and low activation materials. This design utilizes the selfcooled liquid lithium concept for tritium Breeding and the vanadium alloy $\mathrm{V} 4 \mathrm{Cr} 4 \mathrm{Ti}$ for structure. Beryllium, tungsten, tungsten carbide, and $\mathrm{NaK}$ are used in this design to achieve the required breeding and to enhance the shielding performance.

\subsection{Radial Build and Neutron Flux}

The radial build of this design at the midplane is given in Table 3 which lists the thickness, the mix name, the number of fine intervals, the radial extents, the cross section area, and the mass of each zone. There are 28 zones in this model. The zone numbers are used in many figures and this table should be consulted to identify the different zones. Radioactivity calculations have been made for all zones ( 28 zones) and for all the fine intervals (294 intervals) in order to determine accurate spatial distribution for the decay heat and the $\gamma$ source.

Table 4 lists the masses of different materials used in each zone and the total mass of any material in the design. The masses are given in $\mathrm{kg} / \mathrm{cm}$. It should be emphasized that these numbers are based on the simple 1-D model. In order to estimate the total mass of the materials used in ITER, one has to multiply these numbers by an average height of the machine. Also, it should be mentioned that, unlike the SS/Water design, we did not include the outboard magnet in the calculations.

The transport code ONEDANT [3] has been used to calculate the neutron flux. Figure 1 shows a schematic of the midplane radial build-up of ITER Li/V design together with the neutron and photon fluxes. In this figure, for illustrative purpose and in order to show the characteristics of these fluxes and the effects of the different materials on them, all the photon groups are collapsed into only one group, and the neutron groups are 
collapsed into 5 major energy groups whose lower energy limits are $10 \mathrm{MeV}, 1 \mathrm{MeV}, 0.12$ $\mathrm{MeV}, 0.87 \mathrm{eV}$, and $10^{-4} \mathrm{eV}$. More detailed descriptions of the flux are shown in Figures 2 and 3 where isometric views of the inboard and the outboard neutron fluxes are shown as a function of the radius and the lethargy.

Table 3. The radial build of the LiV design.

\begin{tabular}{|l|r|l|r|r|r|r|r|}
\hline Zone & \multicolumn{1}{|c|}{ DR } & mat & intv & \multicolumn{1}{c|}{ R1 } & \multicolumn{1}{c|}{ R2 } & \multicolumn{1}{c|}{ xs-area } & \multicolumn{1}{c|}{ Mass } \\
\hline$\#$ & $\mathrm{~cm}$ & & & $\mathrm{~cm}$ & $\mathrm{~cm}$ & \multicolumn{1}{c|}{$\mathrm{m}^{2}$} & $\mathrm{~kg}$ \\
\hline & 287.50 & vac & 29 & 0.00 & 287.50 & & \\
\hline 1 & 5.00 & SSWP & 5 & 287.50 & 292.50 & 0.91106 & 71.56 \\
\hline 2 & 84.40 & coil & 85 & 292.50 & 376.90 & 17.74917 & 1057.96 \\
\hline 3 & 0.10 & SSWP & 1 & 376.90 & 377.00 & 0.02368 & 1.86 \\
\hline & 13.50 & vac & 14 & 377.00 & 390.50 & & \\
\hline 4 & 5.00 & B4C_Pb & 5 & 390.50 & 395.50 & 1.23465 & 96.45 \\
\hline 5 & 3.00 & Incon & 3 & 395.50 & 398.50 & 0.74833 & 63.16 \\
\hline 6 & 44.50 & WNaK & 44 & 398.50 & 443.00 & 11.76424 & 1489.85 \\
\hline 7 & 3.00 & Incon & 3 & 443.00 & 446.00 & 0.83786 & 70.72 \\
\hline & 5.00 & vac & 5 & 446.00 & 451.00 & & \\
\hline 8 & 7.00 & Vall & 7 & 451.00 & 458.00 & 1.99900 & 121.94 \\
\hline 9 & 5.00 & Li & 5 & 458.00 & 463.00 & 1.44670 & 7.27 \\
\hline 10 & 12.00 & WC & 12 & 463.00 & 475.00 & 3.53618 & 552.57 \\
\hline 11 & 5.00 & Li & 5 & 475.00 & 480.00 & 1.50011 & 7.54 \\
\hline 12 & 0.50 & Vall & 1 & 480.00 & 480.50 & 0.15087 & 9.20 \\
\hline 13 & 6.00 & Li & 6 & 480.50 & 486.50 & 1.82275 & 9.16 \\
\hline 14 & 5.00 & BeZ & 5 & 486.50 & 491.50 & 1.53624 & 28.42 \\
\hline 15 & 4.00 & Li & 4 & 491.50 & 495.50 & 1.24030 & 6.23 \\
\hline 16 & 0.50 & Vall & 1 & 495.50 & 496.00 & 0.15574 & 9.50 \\
\hline & 10.60 & vac & 2 & 496.00 & 506.60 & & \\
\hline & 608.30 & source & 61 & 506.60 & 1114.90 & & \\
\hline & 21.30 & vac & 3 & 1114.90 & 1136.20 & & \\
\hline 17 & 0.50 & Vall & 1 & 1136.20 & 1136.70 & 0.35703 & 21.78 \\
\hline 18 & 4.00 & Li & 4 & 1136.70 & 1140.70 & 2.86187 & 14.38 \\
\hline 19 & 5.00 & BeZ & 5 & 1140.70 & 1145.70 & 3.59147 & 66.44 \\
\hline 20 & 6.00 & Li & 6 & 1145.70 & 1151.70 & 4.33050 & 21.76 \\
\hline 21 & 0.50 & Vall & 1 & 1151.70 & 1152.20 & 0.36190 & 22.08 \\
\hline 22 & 5.00 & Li & 5 & 1152.20 & 1157.20 & 3.62760 & 18.23 \\
\hline 23 & 12.00 & WC & 12 & 1157.20 & 1169.20 & 8.77032 & 1370.46 \\
\hline 24 & 5.00 & Li & 5 & 1169.20 & 1174.20 & 3.68100 & 18.50 \\
\hline 25 & 7.00 & Vall & 7 & 1174.20 & 1181.20 & 5.17980 & 315.97 \\
\hline & 41.50 & vac & 42 & 1181.20 & 1222.70 & & \\
\hline 26 & 3.00 & Incon & 3 & 1222.70 & 1225.70 & 2.30756 & 194.76 \\
\hline 27 & 49.50 & WNaK & 50 & 1225.70 & 1275.20 & 38.89120 & 4925.27 \\
\hline 28 & 3.00 & Incon & 3 & 1275.20 & 1278.20 & 2.40652 & 203.11 \\
\hline Total & & & & & & 123.02365 & 10796.11 \\
\hline
\end{tabular}


Table 4. The materials' masses used in all the zones.

\begin{tabular}{|c|c|c|c|c|c|c|c|c|c|c|c|c|c|c|}
\hline Mat: & ss $3161 \mathrm{w}$ & repxy & cu_ITR & nb3sn & lhe & pb_p & $\mathrm{b} 4 \mathrm{c}$ & incl625 & L_nak68 & WC & $\mathrm{v} 4 \mathrm{cr} 4 \mathrm{ti}$ & L_li & be 100 & total \\
\hline Zone\# & & & & & & & & & & & & & & $\mathrm{Kg} / \mathrm{cm}$ \\
\hline 1 & 71.56 & & & & & & & & & & & & & 71.56 \\
\hline 2 & 655.27 & 44.87 & 186.36 & 167.73 & 3.73 & & & & & & & & & 1057.96 \\
\hline 3 & 1.86 & & & & & & & & & & & & & 1.86 \\
\hline & & & & & & & & & & & & & & \\
\hline 4 & & & & & & 84.01 & 12.45 & & & & & & & 96.45 \\
\hline 5 & & & & & & & & 63.16 & & & & & & 63.16 \\
\hline 6 & & & & & & & & & 19.22 & 1470.63 & & & & 1489.85 \\
\hline 7 & & & & & & & & 70.72 & & & & & & 70.72 \\
\hline & & & & & & & & & & & & & & \\
\hline 8 & & & & & & & & & & & 121.94 & & & 121.94 \\
\hline 9 & & & & & & & & & & & & 7.27 & & 7.27 \\
\hline 10 & & & & & & & & & & 552.57 & & & & 552.57 \\
\hline 11 & & & & & & & & & & & & 7.54 & & 7.54 \\
\hline 12 & & & & & & & & & & & 9.20 & & & 9.20 \\
\hline 13 & & & & & & & & & & & & 9.16 & & 9.16 \\
\hline 14 & & & & & & & & & & & & & 28.42 & 28.42 \\
\hline 15 & & & & & & & & & & & & 6.23 & & 6.23 \\
\hline 16 & & & & & & & & & & & 9.50 & & & 9.50 \\
\hline & & & & & & & & & & & & & & \\
\hline & & & & & & & & & & & & & & \\
\hline & & & & & & & & & & & & & & \\
\hline 17 & & & & & & & & & & & 21.78 & & & 21.78 \\
\hline 18 & & & & & & & & & & & & 14.38 & & 14.38 \\
\hline 19 & & & & & & & & & & & & & 66.44 & 66.44 \\
\hline 20 & & & & & & & & & & & & 21.76 & & 21.76 \\
\hline 21 & & & & & & & & & & & 22.08 & & & 22.08 \\
\hline 22 & & & & & & & & & & & & 18.23 & & 18.23 \\
\hline 23 & & & & & & & & & & 1370.46 & & & & 1370.46 \\
\hline 24 & & & & & & & & & & & & 18.50 & & 18.50 \\
\hline 25 & & & & & & & & & & & 315.97 & & & 315.97 \\
\hline & & & & & & & & & & & & & & \\
\hline 26 & & & & & & & & 194.76 & & & & & & 194.76 \\
\hline 27 & & & & & & & & & 63.54 & 4861.73 & & & & 4925.27 \\
\hline 28 & & & & & & & & 203.11 & & & & & & 203.11 \\
\hline $\mathrm{Kg} / \mathrm{cm}$ & 728.70 & 44.87 & 186.36 & 167.73 & 3.73 & 84.01 & 12.45 & 531.74 & 82.76 & 8255.38 & 500.46 & 103.07 & 94.86 & 10796.11 \\
\hline
\end{tabular}




\subsection{Fine Mesh Results}

As previously indicated, calculations have been made using fine mesh in order to determine accurately the $\gamma$ source distribution to be used for the contact dose calculations. Also, for any serious LOCA analysis, the spatial distribution of the decay heat is required. Figures 4-7 show the results of these calculations for the radioactivity, the decay heat, the air-BHP, and the point-source contact dose, respectively. The latter is based on the dose at one meter radius in the air from a point $\gamma$ source which is taken as the specific $\gamma$ intensity at each interval. Thus, these $\gamma^{\text {ss }}$ do not suffer any attenuation and the resultant dose is useful only, in the context of a large system, in identifying the spatial distribution of the $\gamma$ source intensity in the system.

As seen in these figures, there are orders of magnitude attenuation of the different responses within thick zones. This indicates that using the average flux in a thick zone to calculate the radioactivity of that zone would underestimate the activity level in the zone. 

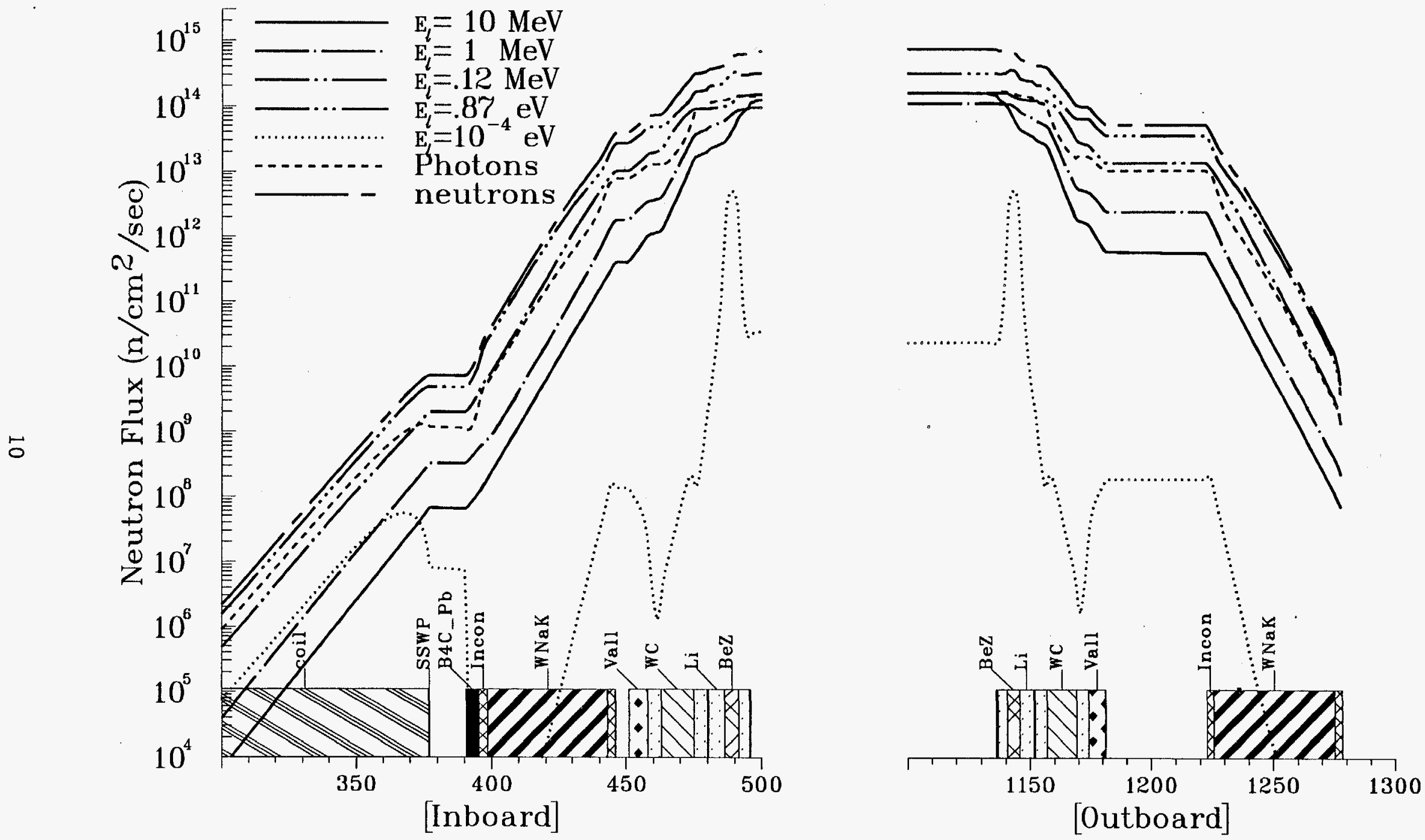

Radius (cm)

Figure 1. The midplane radial build of ITER, the neutron, and the photons fluxes ( $\mathrm{Li} / \mathrm{V}$ design). 


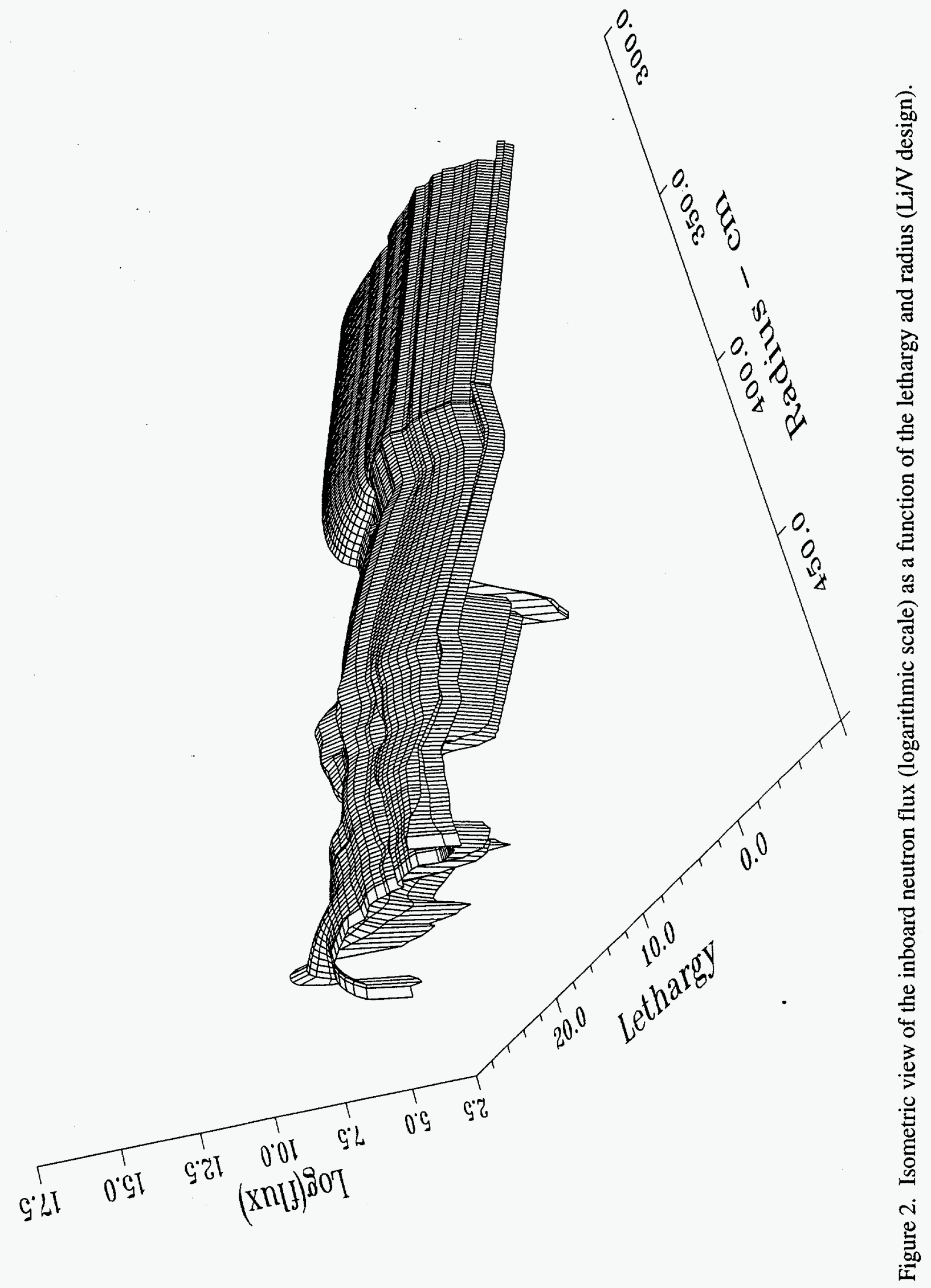




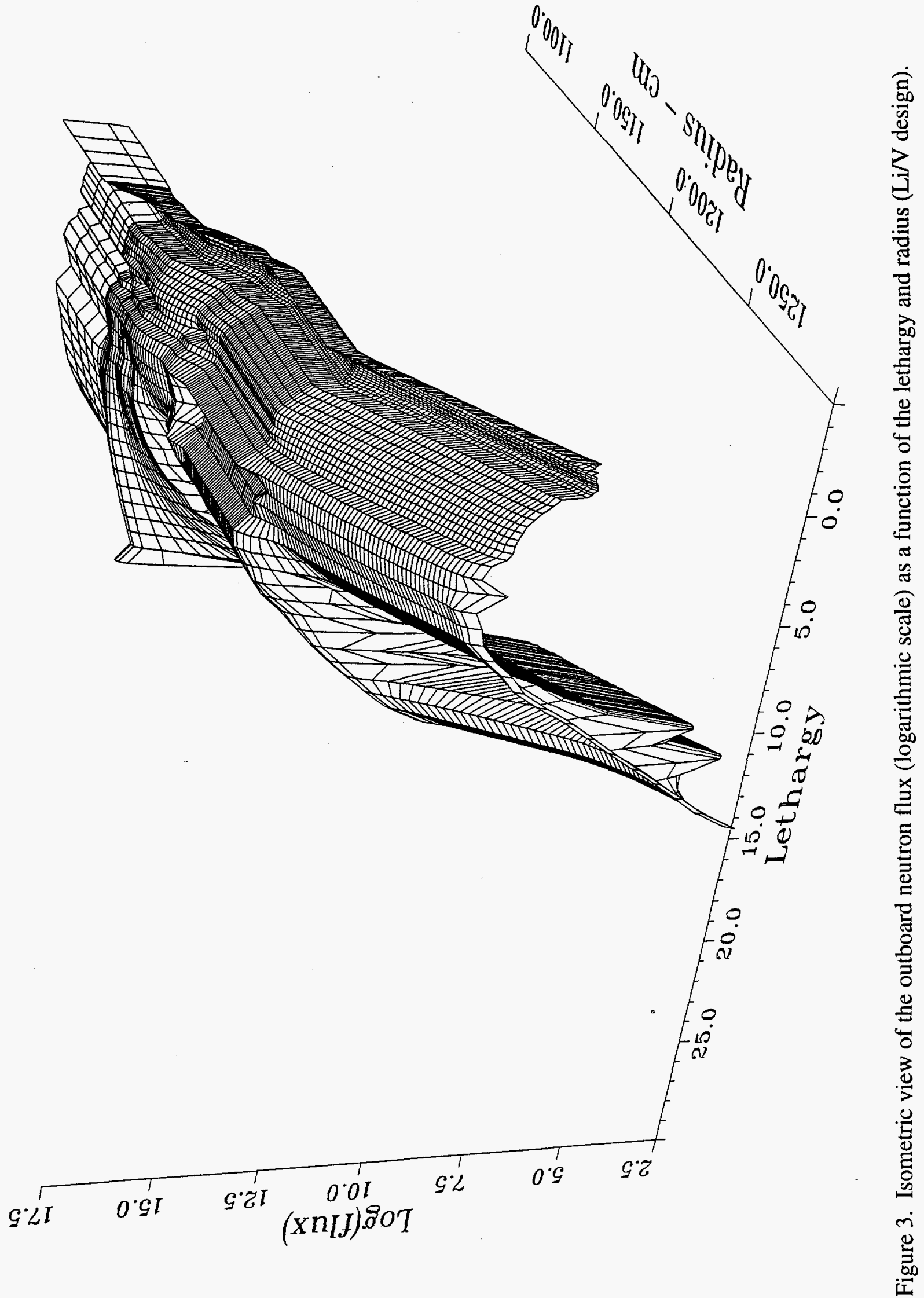



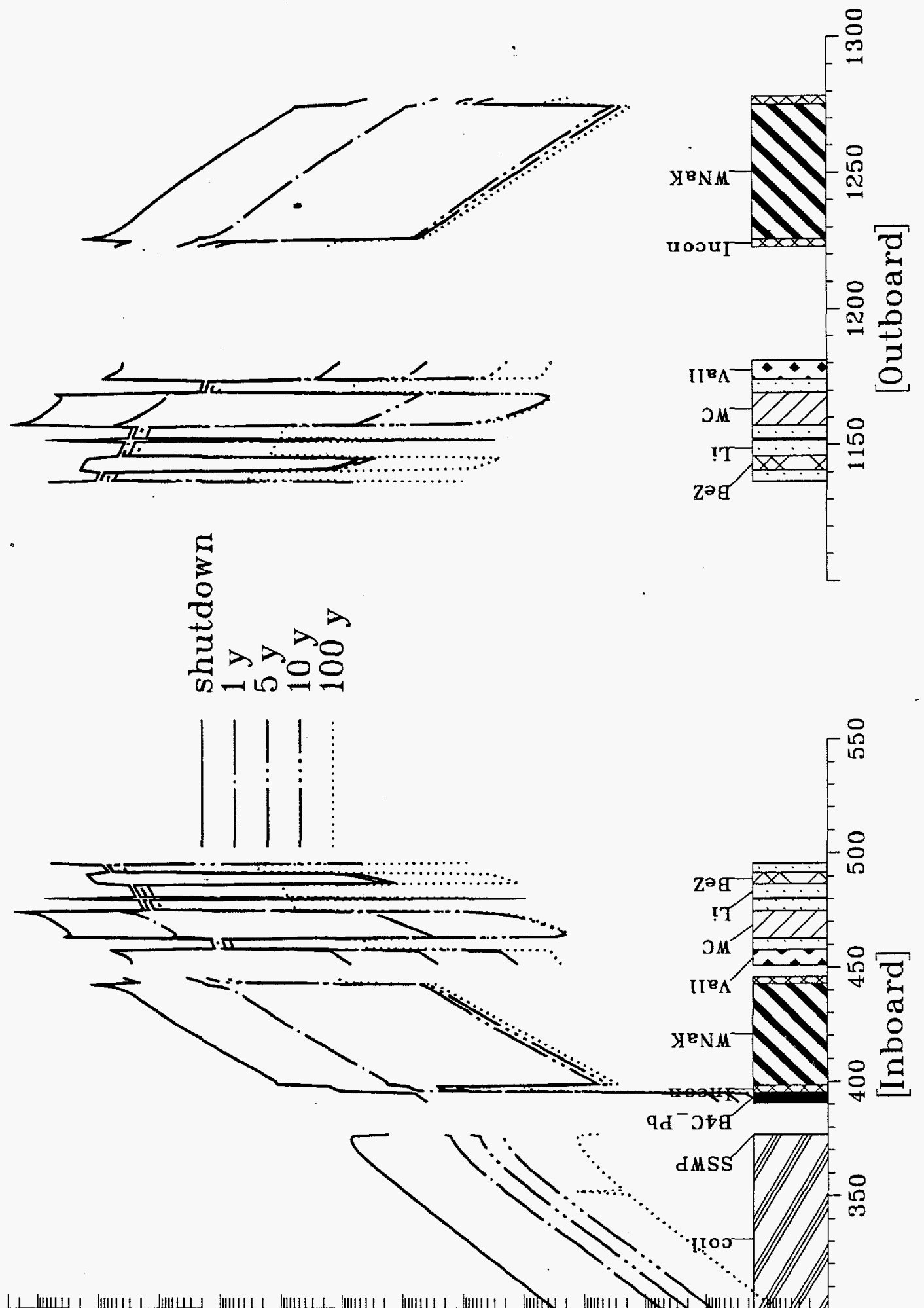

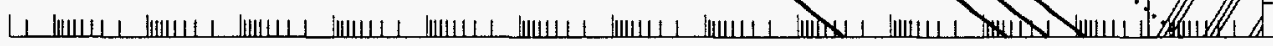

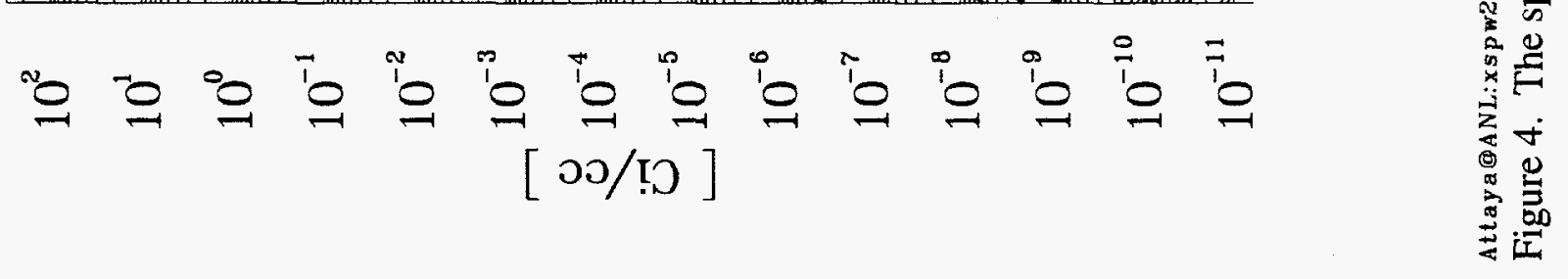




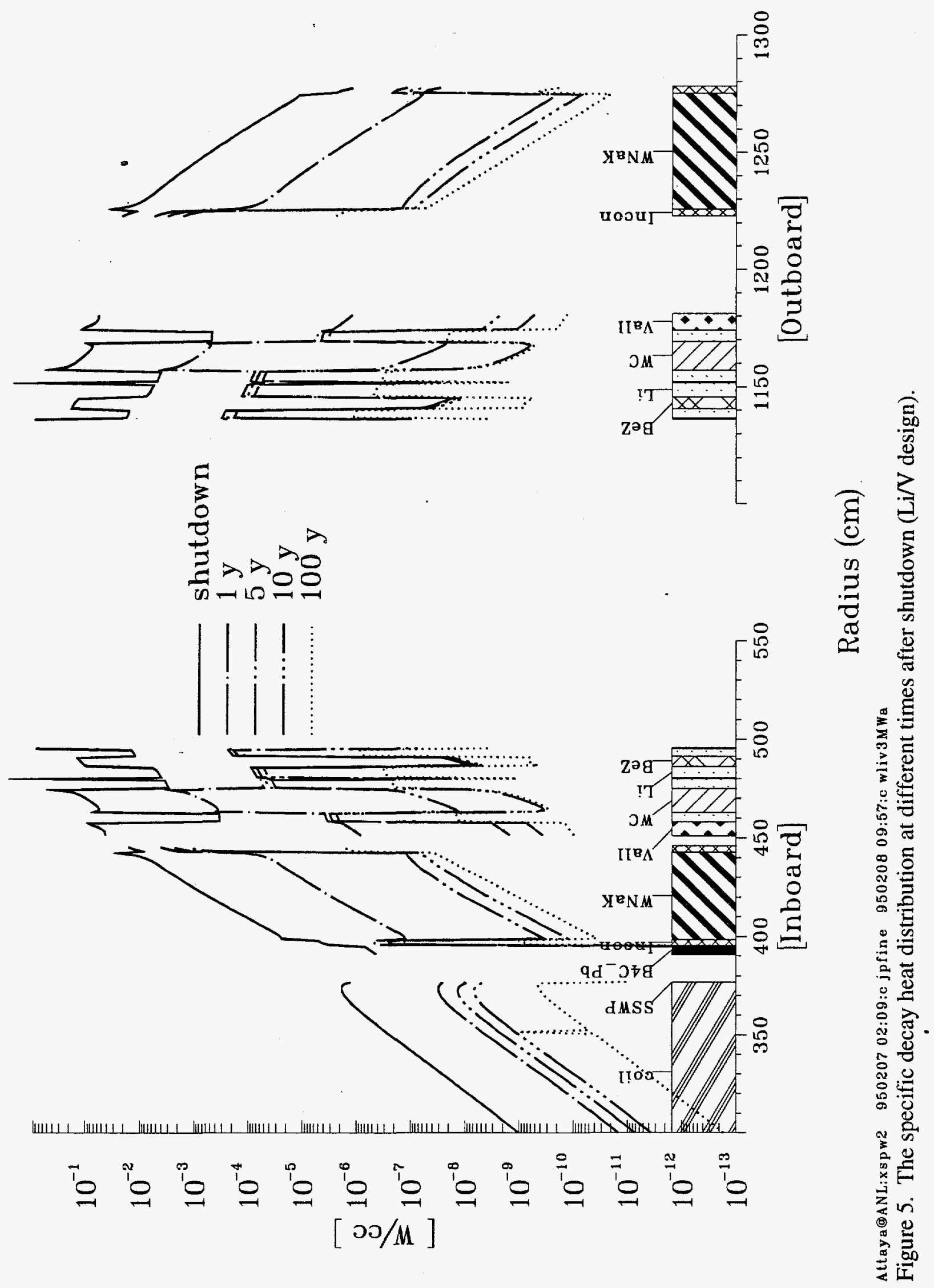



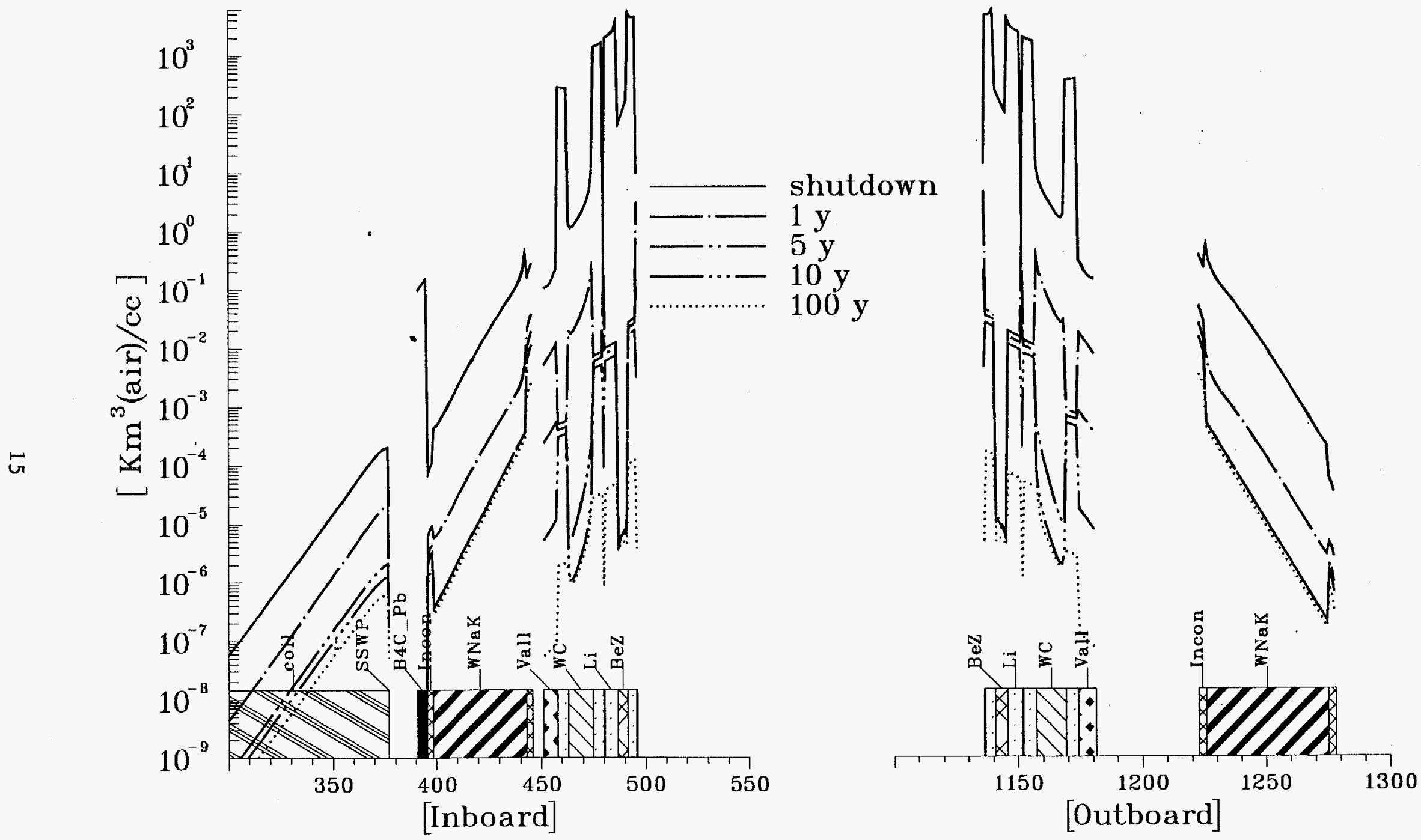

Radius (cm)

Attaya@ANL:xspw2 950207 02:09:c jpfine 950208 09:58:c wliv3MWa

Figure 6. The specific air-BHP distribution at different times after shutdown ( $\mathrm{Li} / \mathrm{V}$ design). 


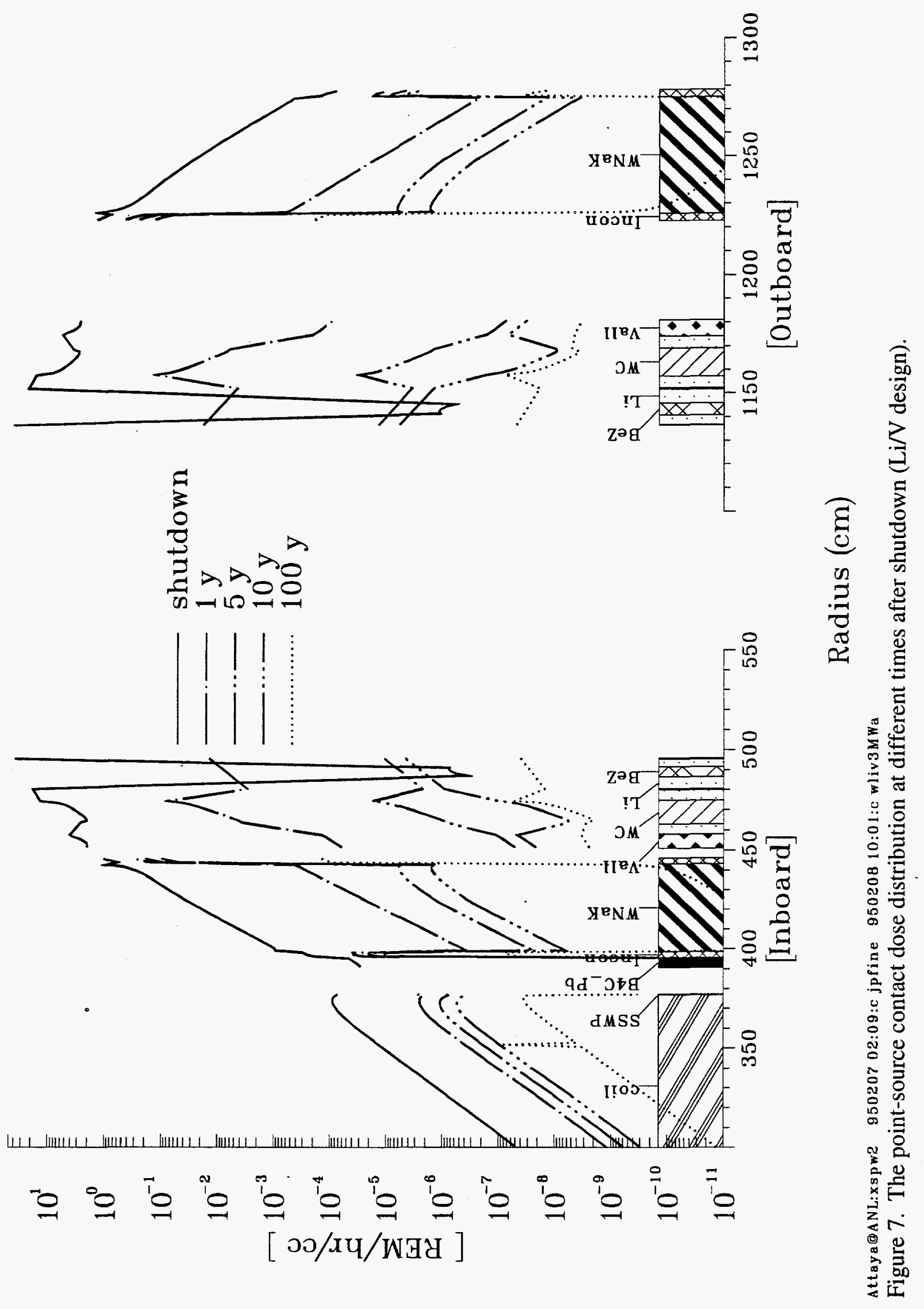




\subsection{System Analysis}

The total radioactivity, the total decay heat, and the total air biological hazard potential of this design after operating up to a neutron fluence of $3 \mathrm{Mwa} / \mathrm{m}^{2}$ are shown as a function of time after shutdown in Figs. 8-10. The inhalation biological hazard potential (BHP $\left.{ }_{\text {air }}\right)$ for the public, is based on the US Federal Regulations (10CFR20).

There is about $15 \mathrm{MCi} / \mathrm{cm}$ after the shutdown. The isotopic contributions to this activity are shown in the top part of the figure and the zonal contributions (see Table 3) are shown in the bottom part. In this figure and similar ones, only isotopes or zones that contribute more than $10 \%$ to the total response, at any time, are shown. The shadowed area at the top represents the contributions of all other isotopes or zones not shown in the figure.

In this design, the radioactivity is dominated by the WC zones (No. 10, 23) for about one year. Afterward and discarding the activity of the lithium zones, the radioactivity is primarily due to the vacuum vessel (VV) which is designed of the Inconel alloy (zones 5, 7, 26, and 28). In the calculations, it is assumed that the tritium produced in the Li's zones is not removed. Because of the production of the ${ }^{10} \mathrm{Be}$ isotope, which has $1.5110^{6}$ years half life, in the beryllium zones (No. 14, and 19), these zones are the dominant contributors to the very long-term radioactivity.

At shutdown, there is about $40 \mathrm{~kW} / \mathrm{cm}$ of radiation power. About 40 to $90 \%$ of this power is produced by photons. Usually, in the LOCA analyses [4,5], the decay heat is assumed localized where it is produced. This leads to a large concentration of heat that in turn produces hot spots. The fact that a large fraction of this heat is generated by photons, which have larger range than the charged particles, may level the heat concentration throughout the system. The decay heat is also dominated by the WC zones 
and the Inconel zones. The contributions of the vanadium alloy to the total decay heat is about $18 \%$ and last only for less than one hour after shutdown.

The $\mathrm{BHP}_{\text {air }}$ is dominated by the WC and the Inconel zones except in the very short time after shutdown, where it is dominated by the $\mathrm{Be}$ and $\mathrm{Li}$ zones because of the production of ${ }^{8} \mathrm{Li}$ and ${ }^{6} \mathrm{He}$. 

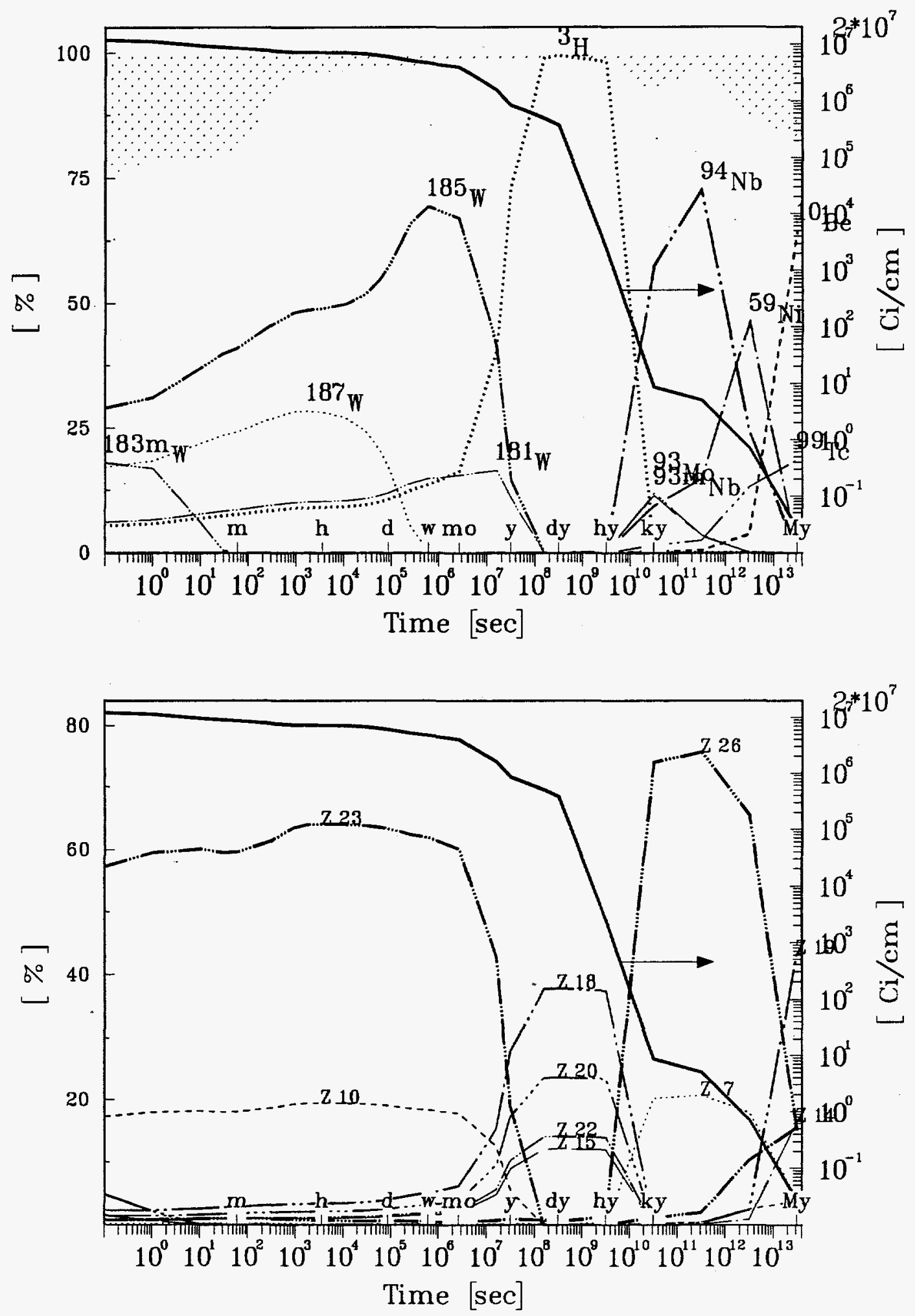

Figure 8 . The total radioactivity (per $\mathrm{cm}$ height) in the system, top: isotopic contributions, bottom: zonal contributions (Li/V design). 

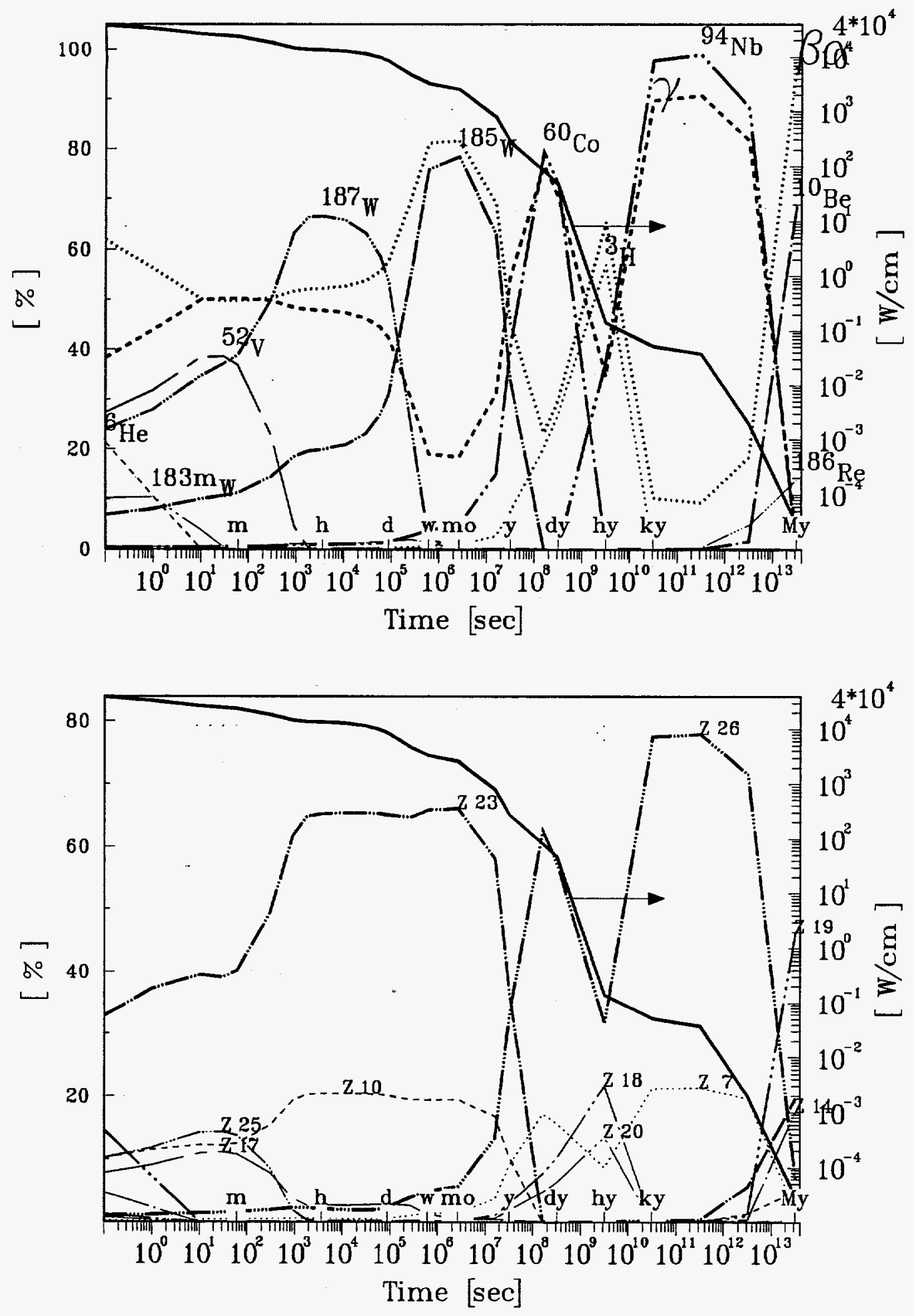

0

Figure 9. The total decay heat (per $\mathrm{cm}$ height) in the system, top: isotopic contributions, bottom: zonal contributions ( $\mathrm{Li} / \mathrm{V}$ design). 

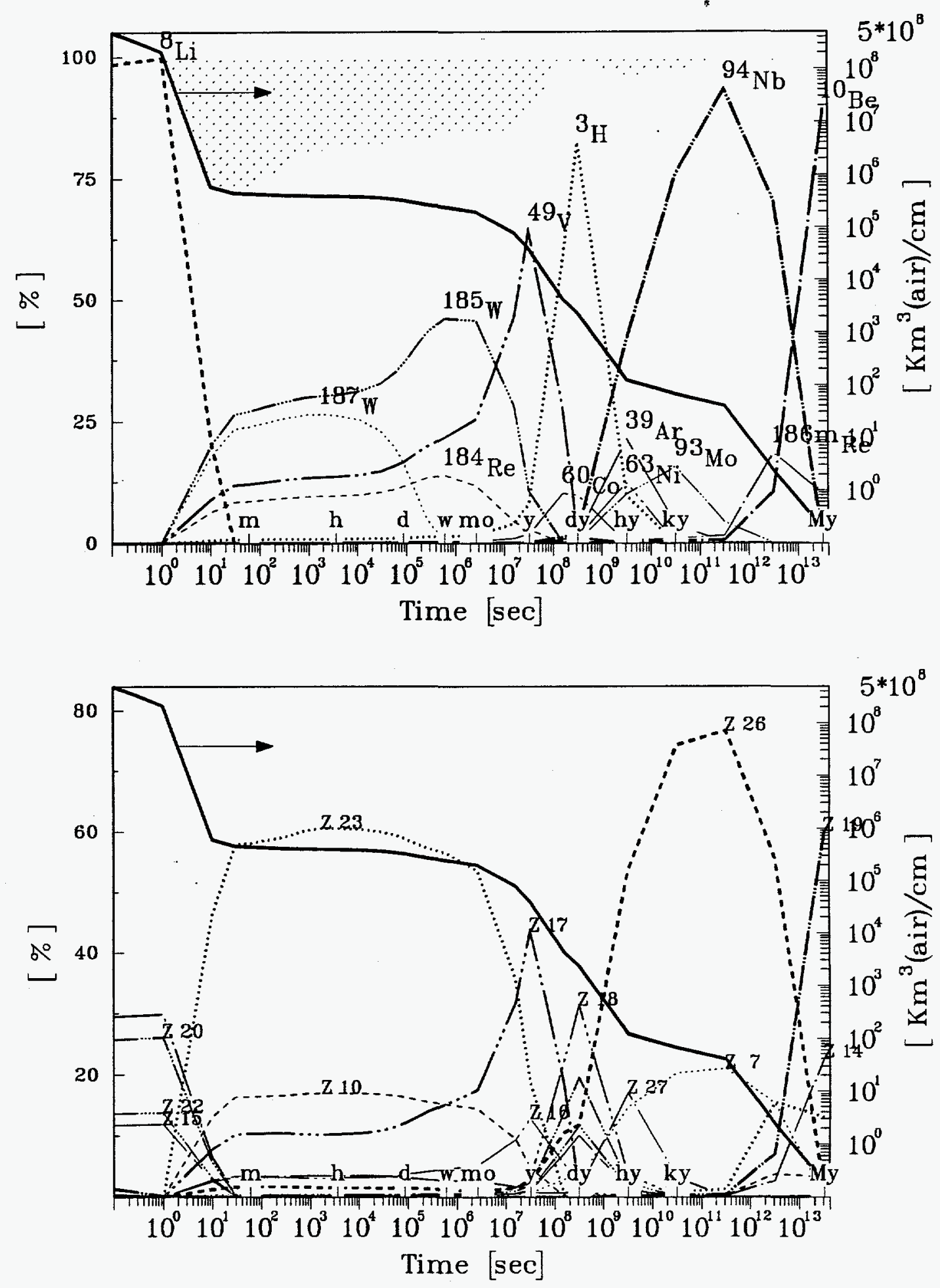

Figure 10. The total air BHP (per $\mathrm{cm}$ height) in the system, top: isotopic contributions, bottom: zonal contributions ( $\mathrm{Li} / \mathrm{V}$ design). 


\subsection{Contact Dose}

The $\gamma$ sources have been calculated for all the fine mesh and for two neutron fluences of $3 \mathrm{MWa} / \mathrm{m}^{2}$ and $0.1 \mathrm{MWa} / \mathrm{m}^{2}$ and for different times after shutdown. The $\gamma$ sources, at each time step after shutdown are then used in the ONEDANT code to calculate the $\gamma$ flux and the dose equivalent rate everywhere in the reactor.

Figure 11 shows the dose distribution after the $3 \mathrm{Mwa} / \mathrm{m}^{2}$ fluence. The effect of the Inconel on the dose is evident. The four peaks in this figure are due to the Inconel. To underscore this effect, another $\gamma$ transport calculation was made without the Inconel $\gamma$ source and the results are shown in Fig. 12. Figure 13 shows the dose due only the vanadium alloy's $\gamma$ source

Figure 14 shows the dose distribution after the $0.1 \mathrm{MWa} / \mathrm{m}^{2}$ fluence. The recommended dose rate limit of $2.5 \mathrm{mrem} / \mathrm{hr}$ for a full time worker can not be achieved anywhere for a long time after shutdown. 


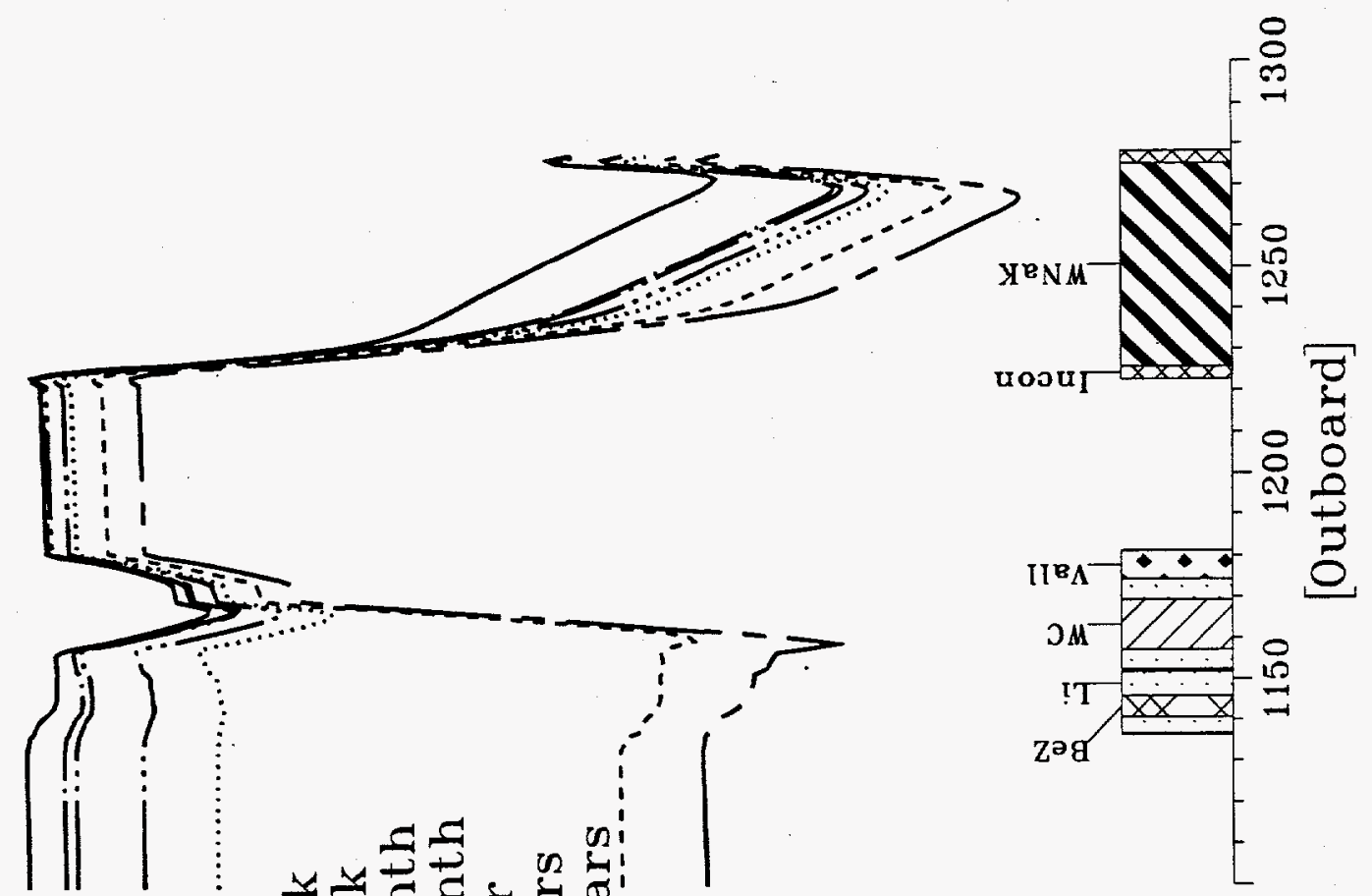

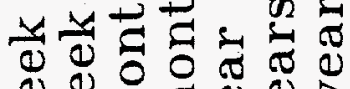

(1) O $0 \circlearrowright ⿻$

₹

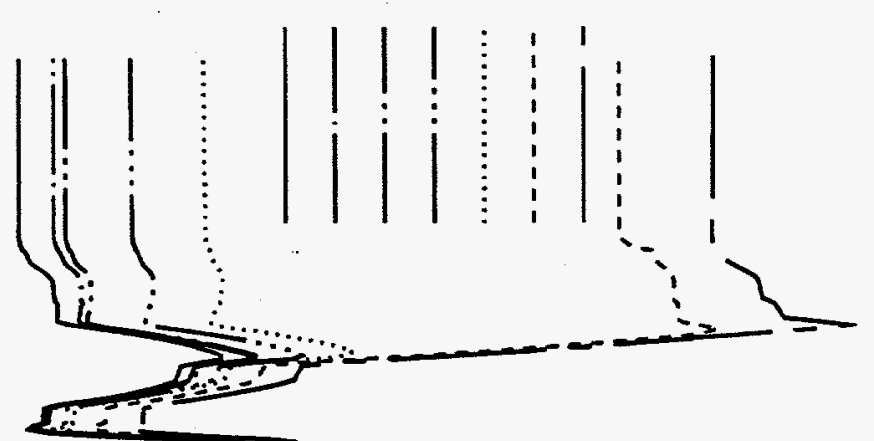

$-10$

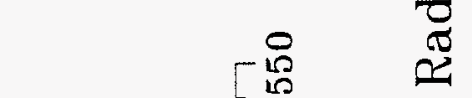

$\dot{s}$

है

$\infty$

ते

mi

氖

$\overline{\mathrm{E}}$

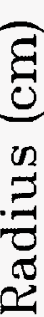

递

$\stackrel{\mathscr{\Xi}}{\Xi}$

苛

$\stackrel{5}{\square}$

농

กี

]

ธิ์

$\frac{\pi}{2}$

焉

용

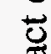

ฮํำ

Е

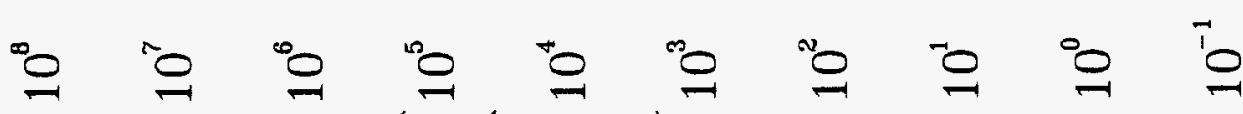

(хч/uәxш) วsod

文 


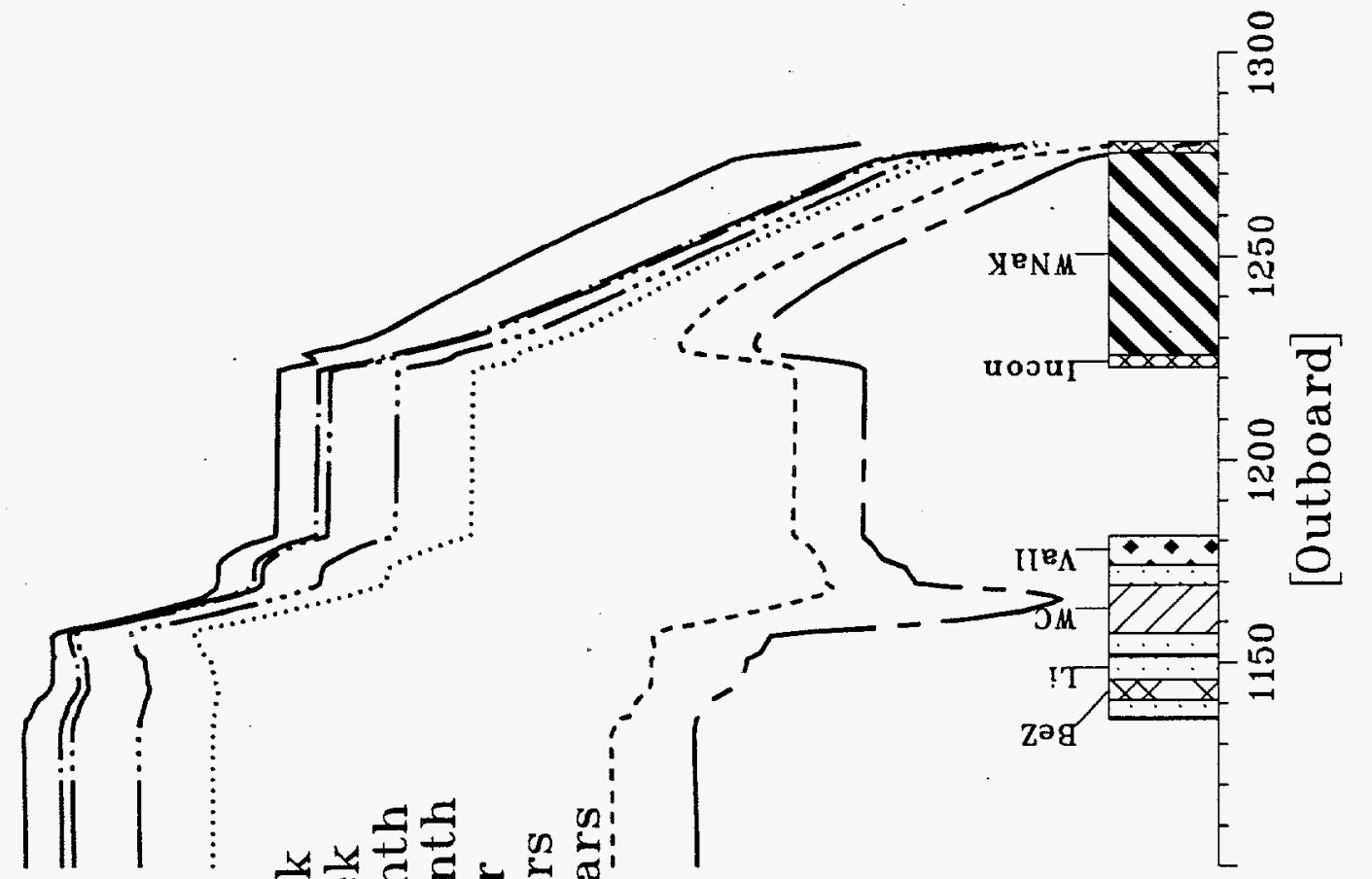

녕

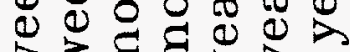

₹ $\xi E \lambda>$

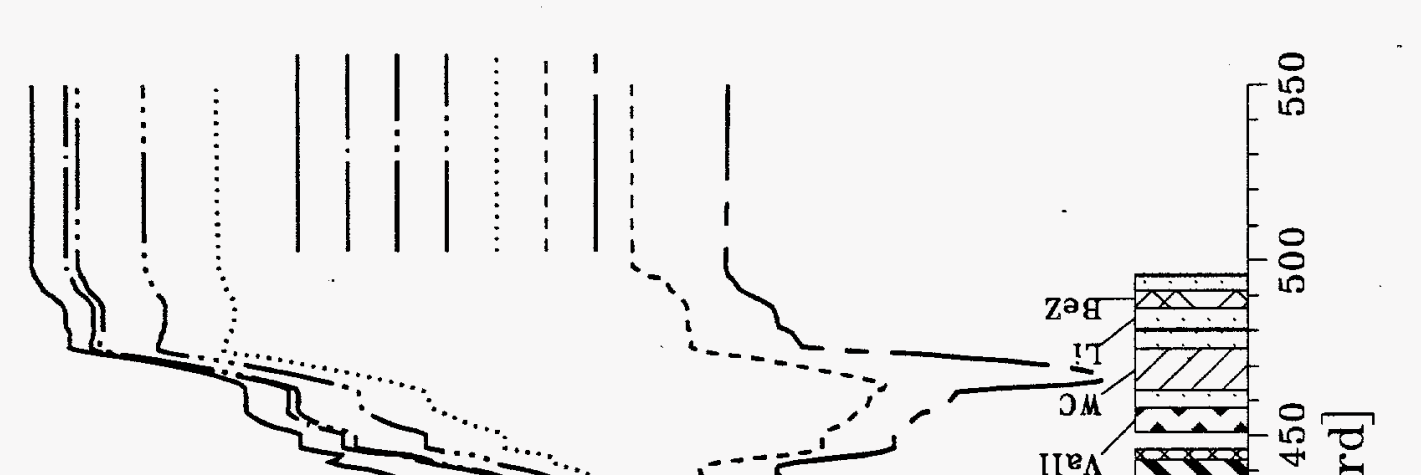

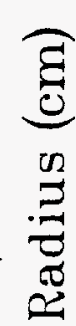

0.

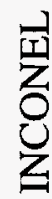

$\sum^{\infty}$

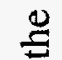

플

焉

ญ̊

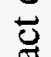

్ㅡㅇ

E

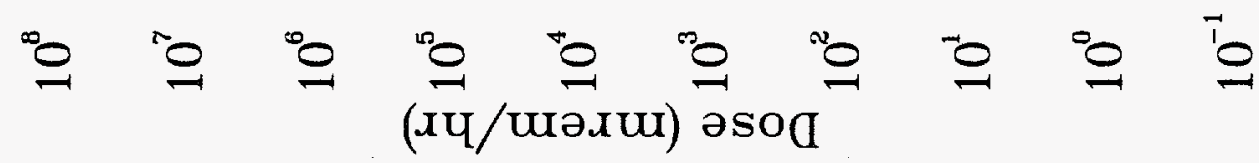

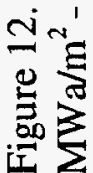



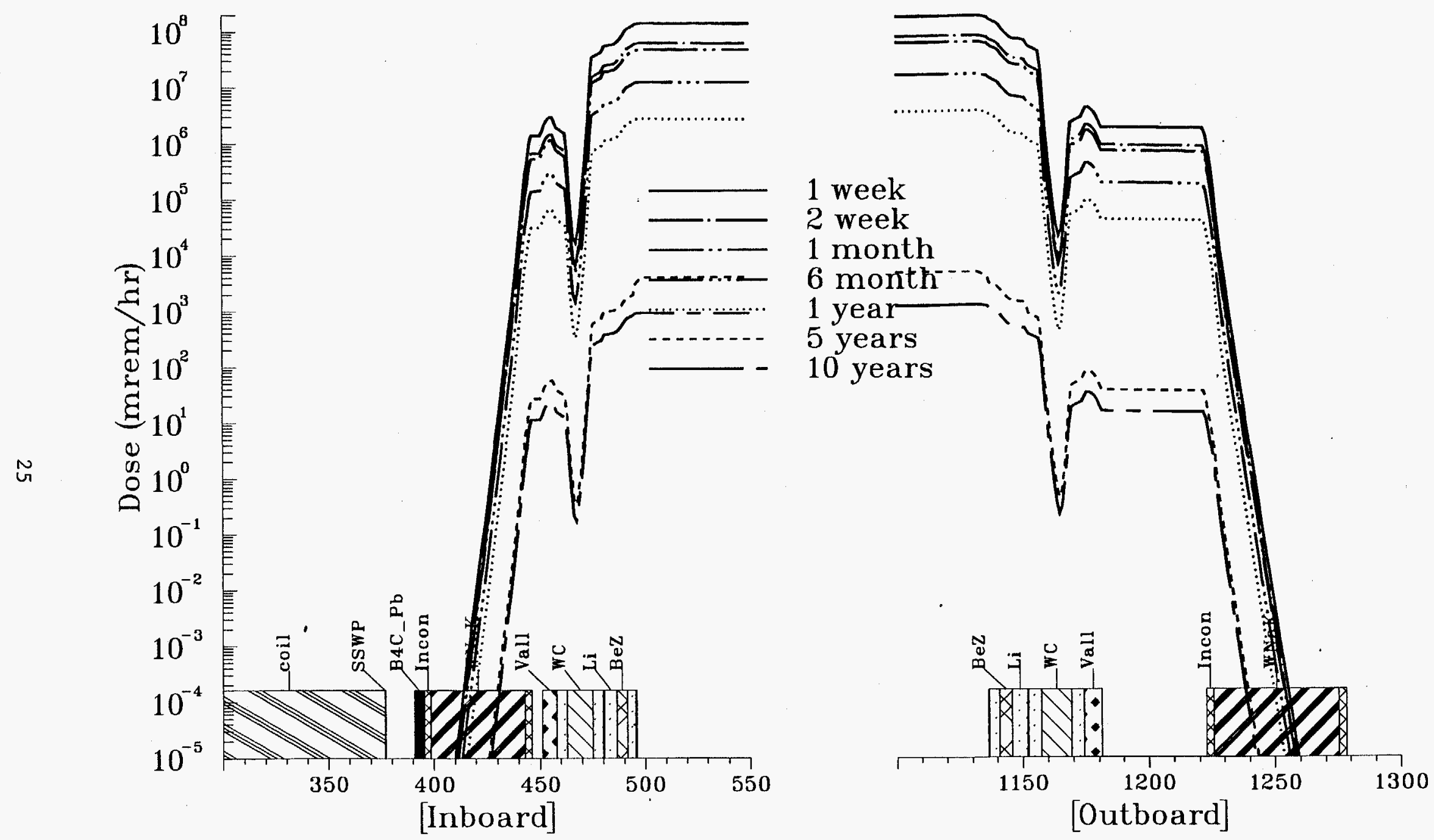

\section{Radius (cm)}

Figure 13. The contact dose, with only the vanadium photon source, at different times after shutdown and after 3.29 FPYs (3 $\left.\mathrm{MWa} / \mathrm{m}^{2}-\mathrm{Li} / \mathrm{V}\right)$. 


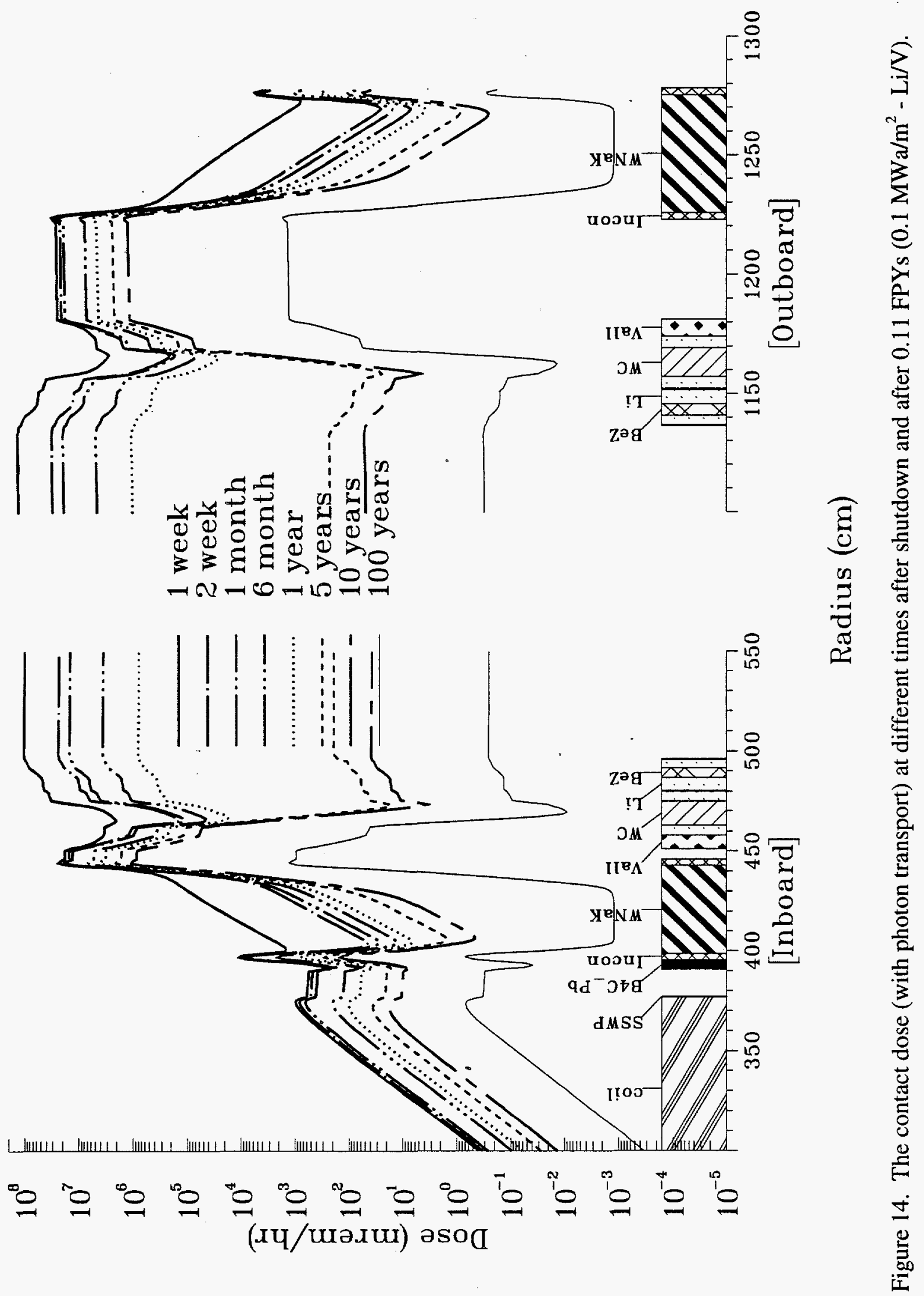




\subsection{Detailed Zones' Results}

For each zone in the design, the radioactivity, the decay heat, the $\mathrm{BHP}_{\text {air }}$, and the point-source contact dose are shown in Appendix A. The zones are ordered (in Appendix A) from the front (plasma side) to the back of the inboard and the outboard shields respectively.

\section{Summary and Conclusions}

Detailed activation analysis for ITER has been performed as a part of ITER Task D4. The calculations have been performed for the shielding blanket (SS/water) and for the breeding blanket $(\mathrm{Li} / \mathrm{V})$ options. The activation code RACC-P, which has been modified under ITER Task-D-10 for pulsed operation, has been used in this analysis. The spatial distributions of the radioactive inventory, decay heat, biological hazard potential, and the contact dose were calculated for the two designs for different operation modes and targeted fluences.

A one-dimensional toroidal geometrical model has been utilized to determine the neutron fluxes in the two designs. The results are normalized for an inboard and outboard neutron wall loadings of 0.91 and $1.2 \mathrm{MW} / \mathrm{m}^{2}$, respectively.

The point-wise distributions of the decay gamma sources have been calculated everywhere in the reactor at several times after the shutdown of the two designs and are then used in the transport code ONEDANT to calculate the biological dose everywhere in the reactor. The point-wise distributions of all the responses have also been calculated. These calculations have been performed for neutron fluences of $3.0 \mathrm{MWa} / \mathrm{m}^{2}$, which corresponds to the target fluence of ITER, and $0.1 \mathrm{MWa} / \mathrm{m}^{2}$, which is anticipated to be the beginning of an extended maintenance period.

The decay heat results show a large fraction of this energy (50 to 90\%) is produced by photons. This implies that this energy would be transported to different parts 
of the reactors, relieving the energy concentration at high intensity source locations such as the first wall. Accurate modeling for the decay gamma transport is required to produce realistic spatial distribution of the decay heat which may be used in LOCA and LOFA analyses.

The radioactivity, the decay heat, and the contact dose of the $\mathrm{Li} / \mathrm{V}$ design is dominated by the WC and the Inconel alloy. 
Appendix A

Li/V Design

Detailed Zones' Results 

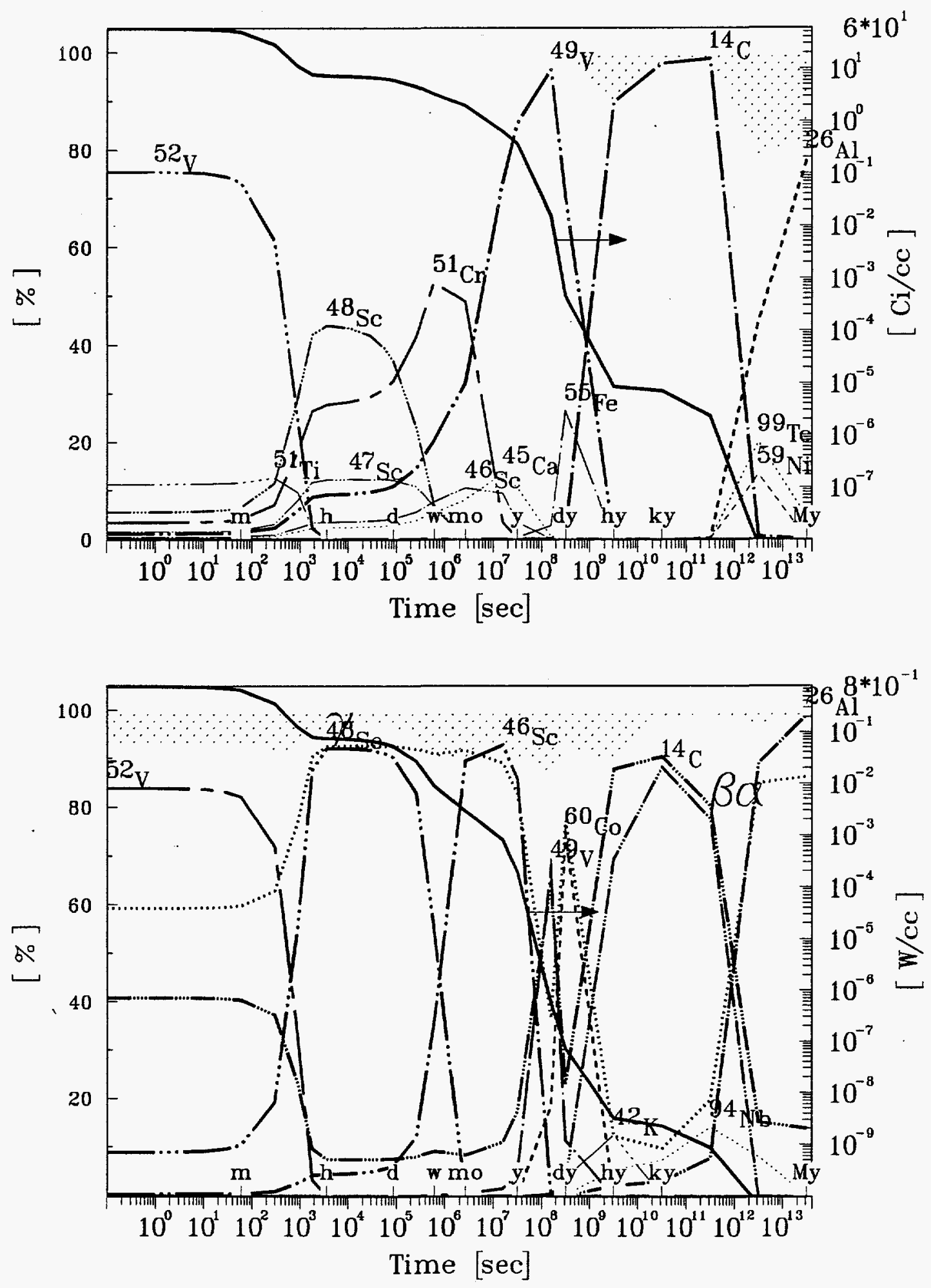

Figure 15. The specific radioactivity (top) and the specific decay heat (bottom) in zone \# 16 (I_Vall - Li/V). 

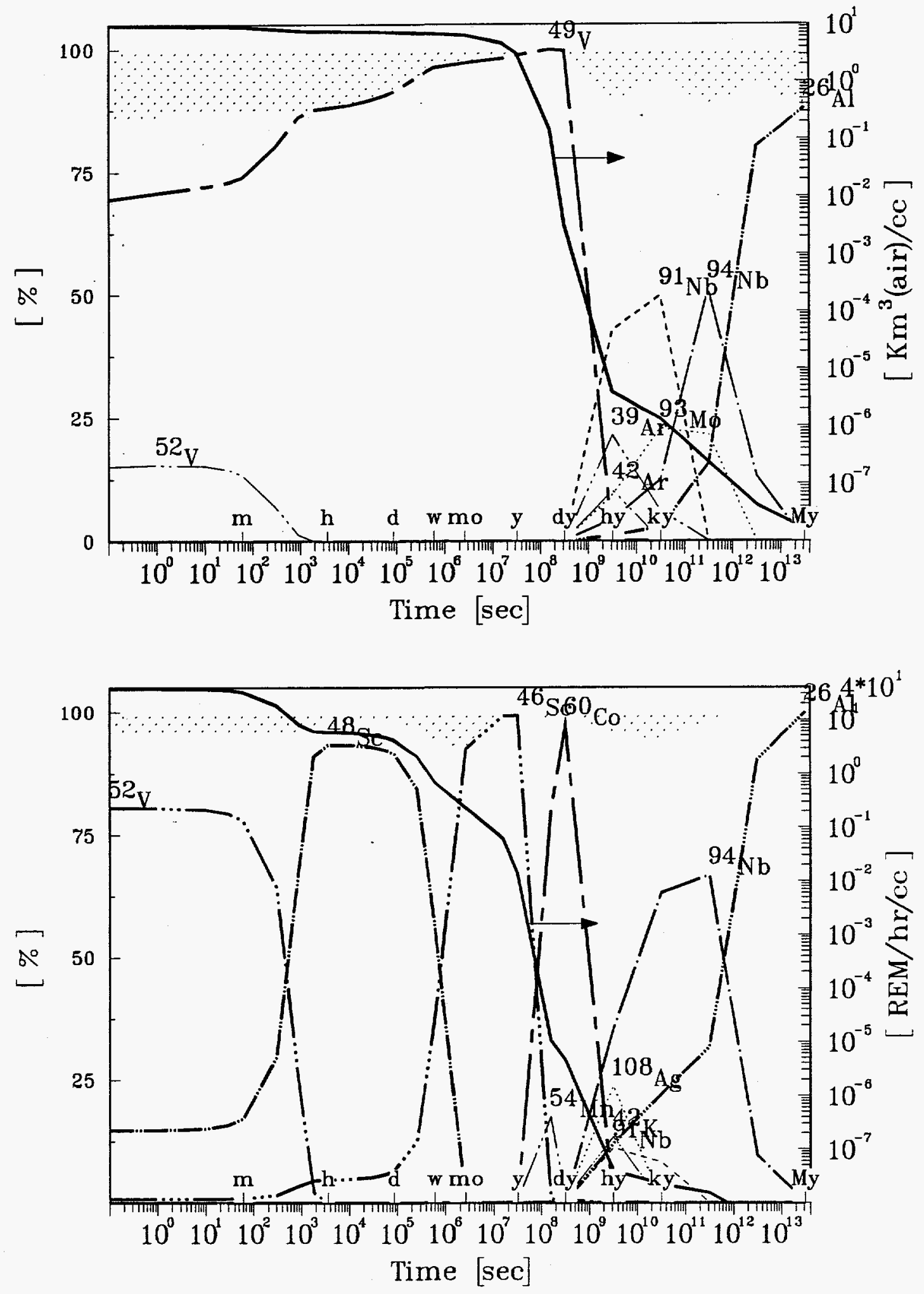

Figure 16. The specific air-BHP (top) and the point-source contact dose (bottom) in zone \# 16 (I_Vall - Li/V). 

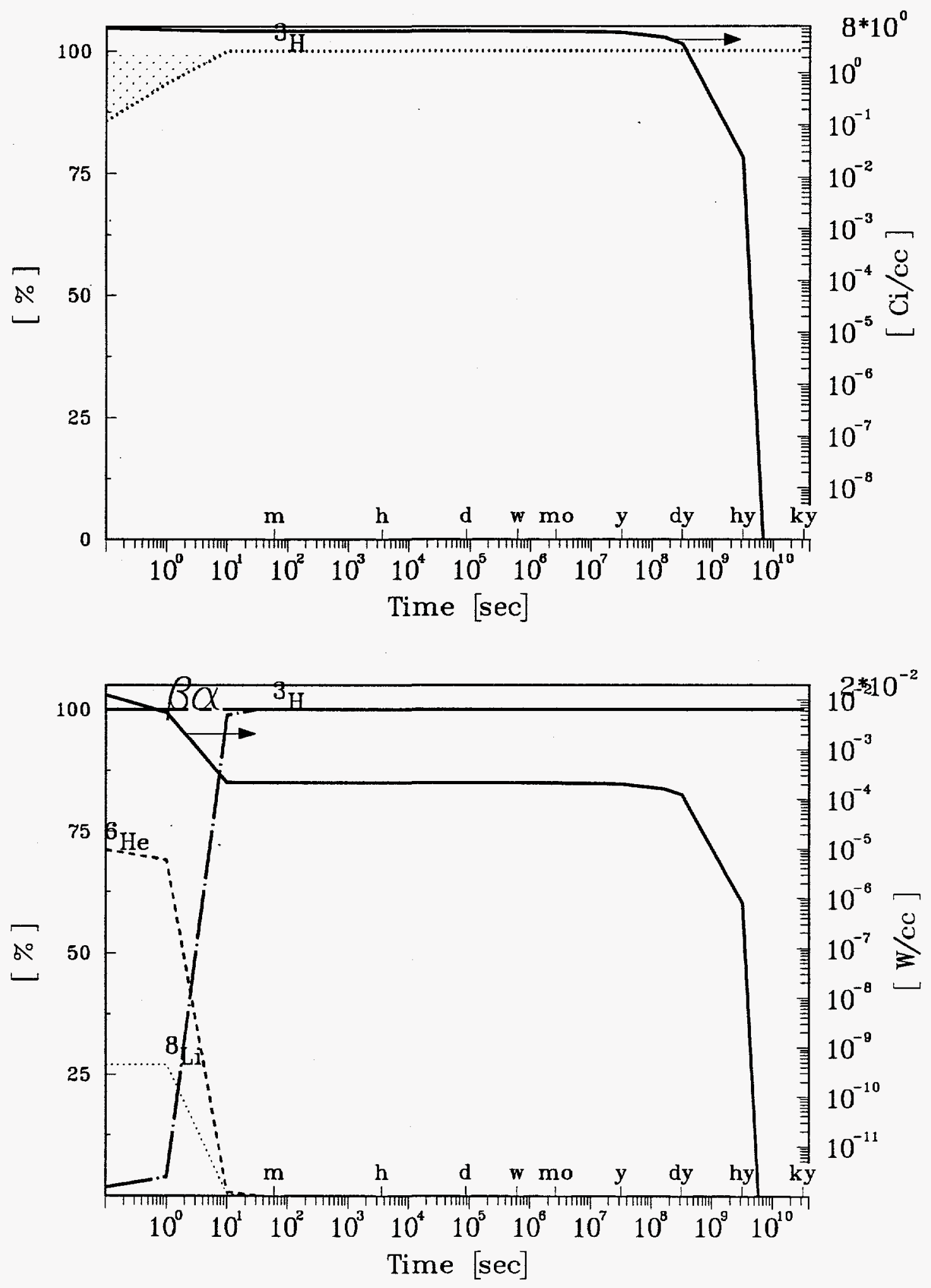

Figure 17. The specific radioactivity (top) and the specific decay heat (bottom) in zone \# 15 (I_Li - Li/V). 


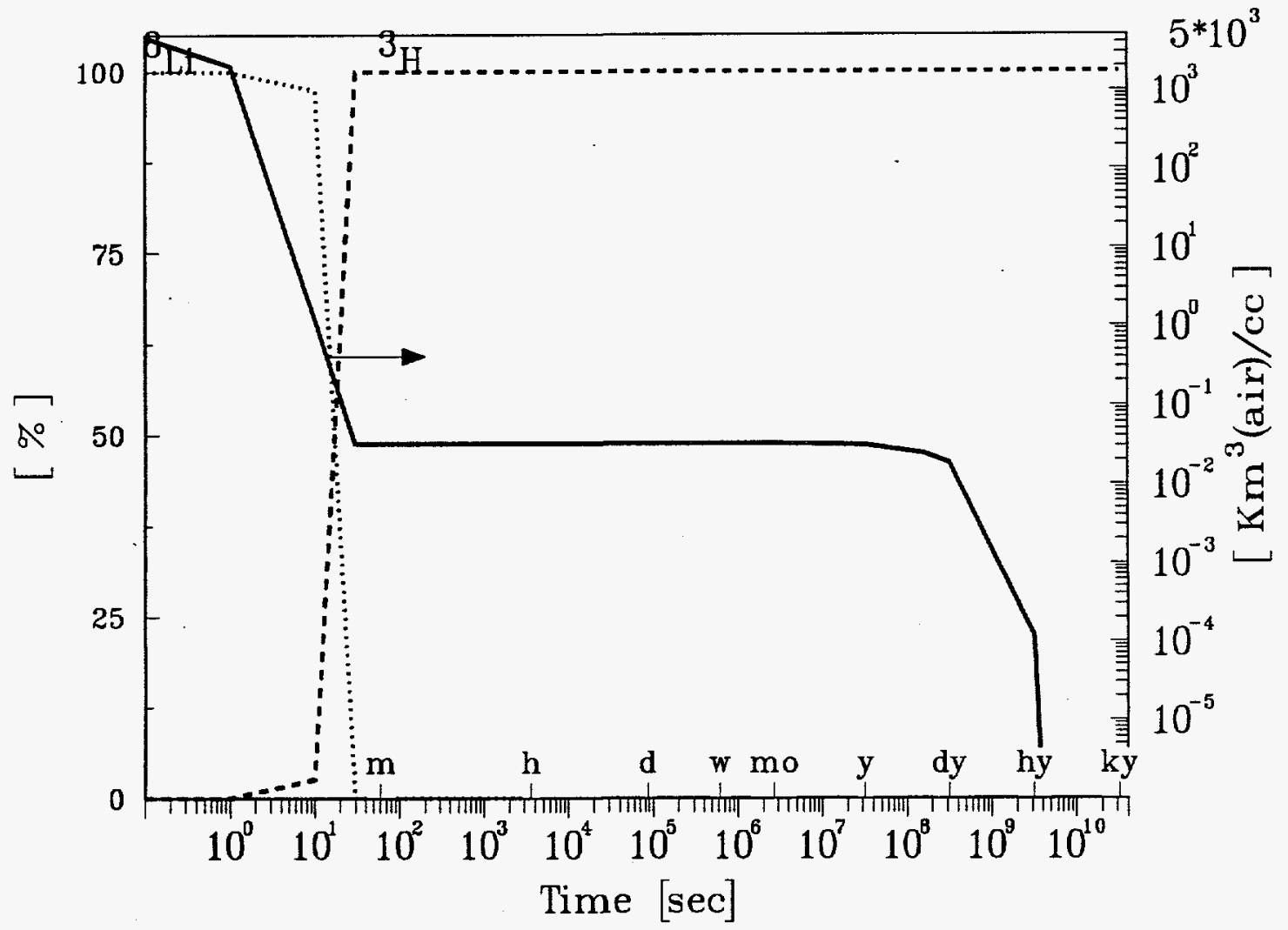

Figure 18. The specific air-BHP in zone \# $15\left(\mathrm{I} \_\mathrm{Li}-\mathrm{Li} / \mathrm{V}\right)$. 

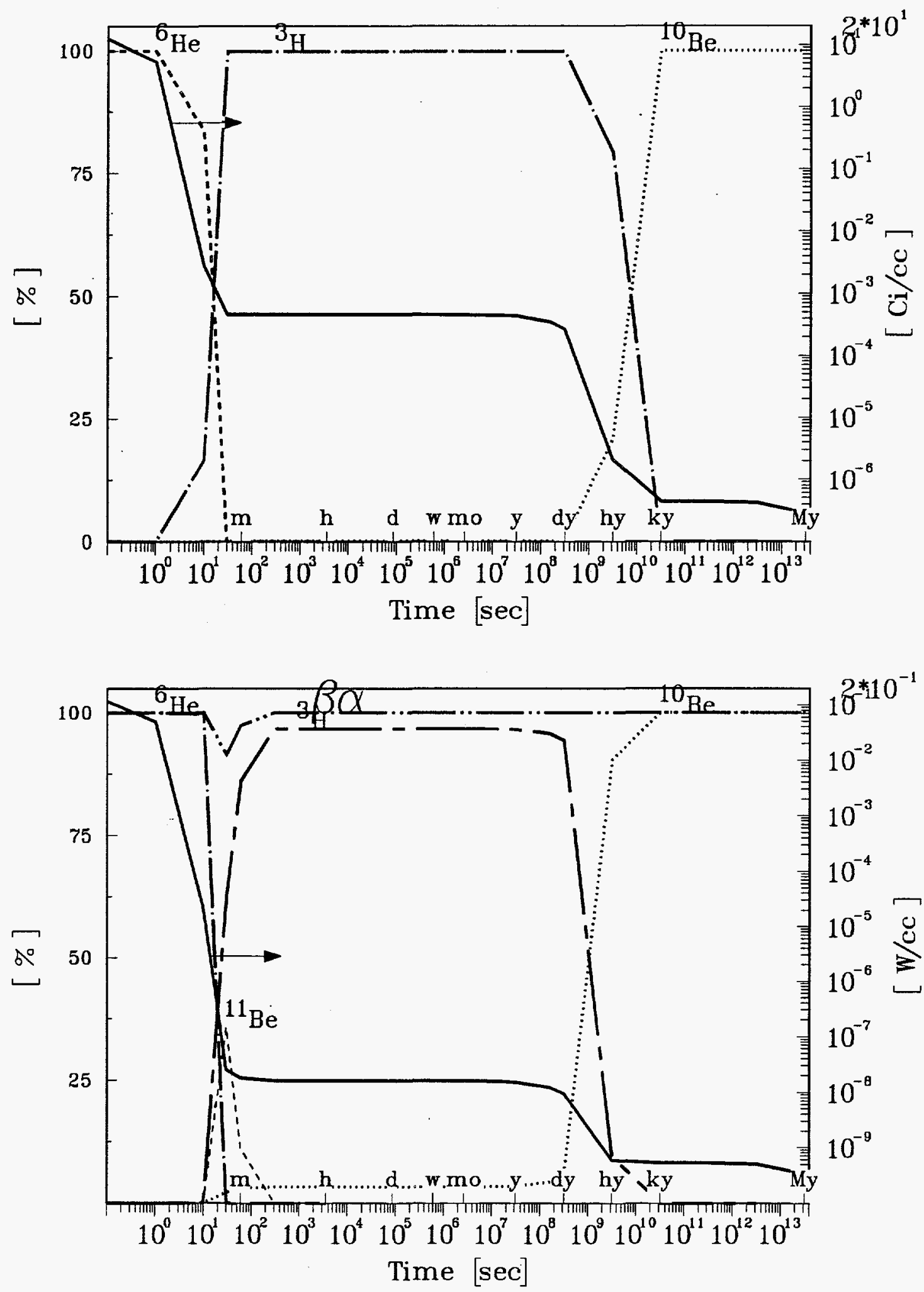

Figure 19. The specific radioactivity (top) and the specific decay heat (bottom) in zone \# 14 (I_BeZ - Li/V). 

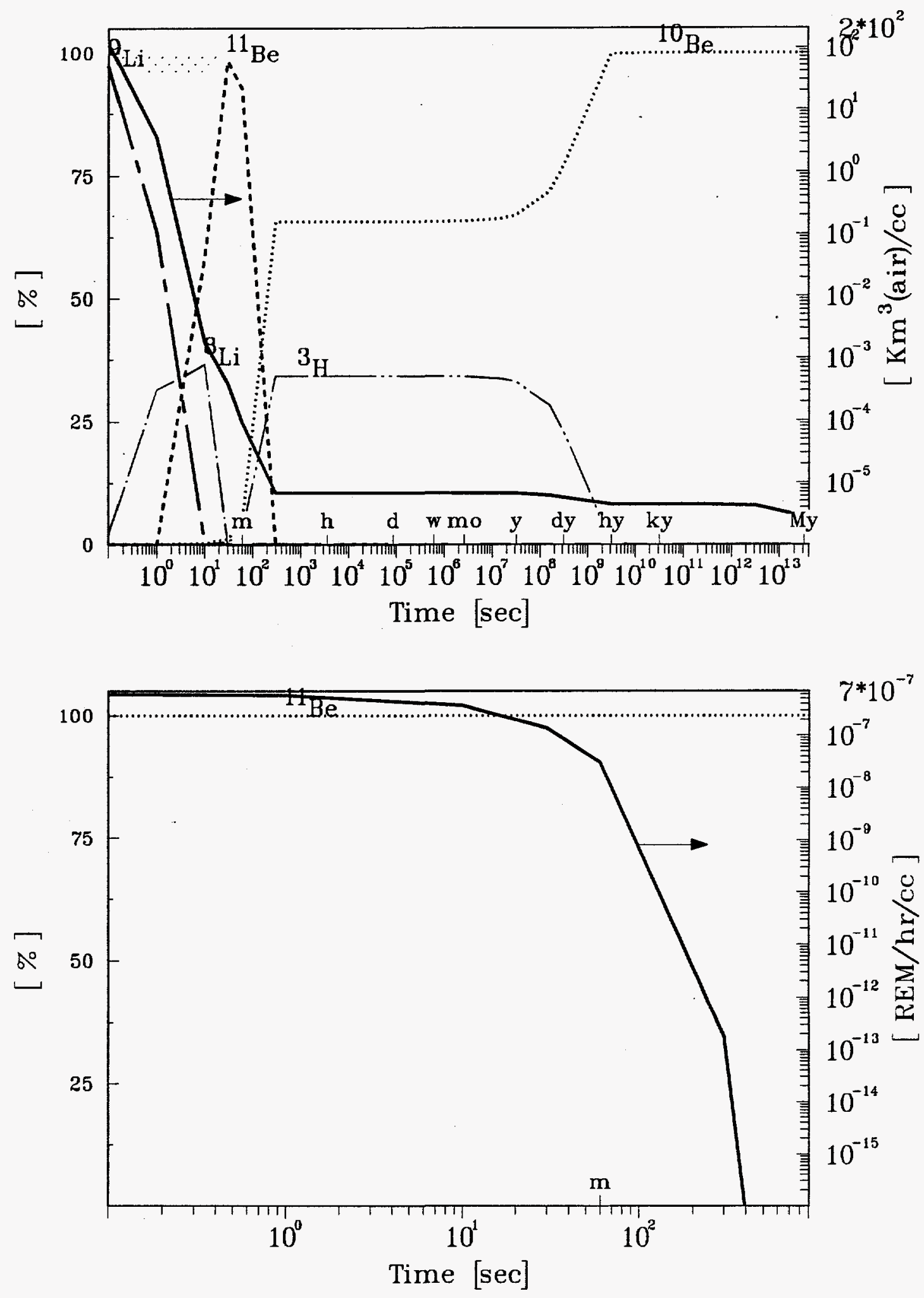

Figure 20. The specific air-BHP (top) and the point-source contact dose (bottom) in zone \# 14 (I_BeZ - Li/V). 

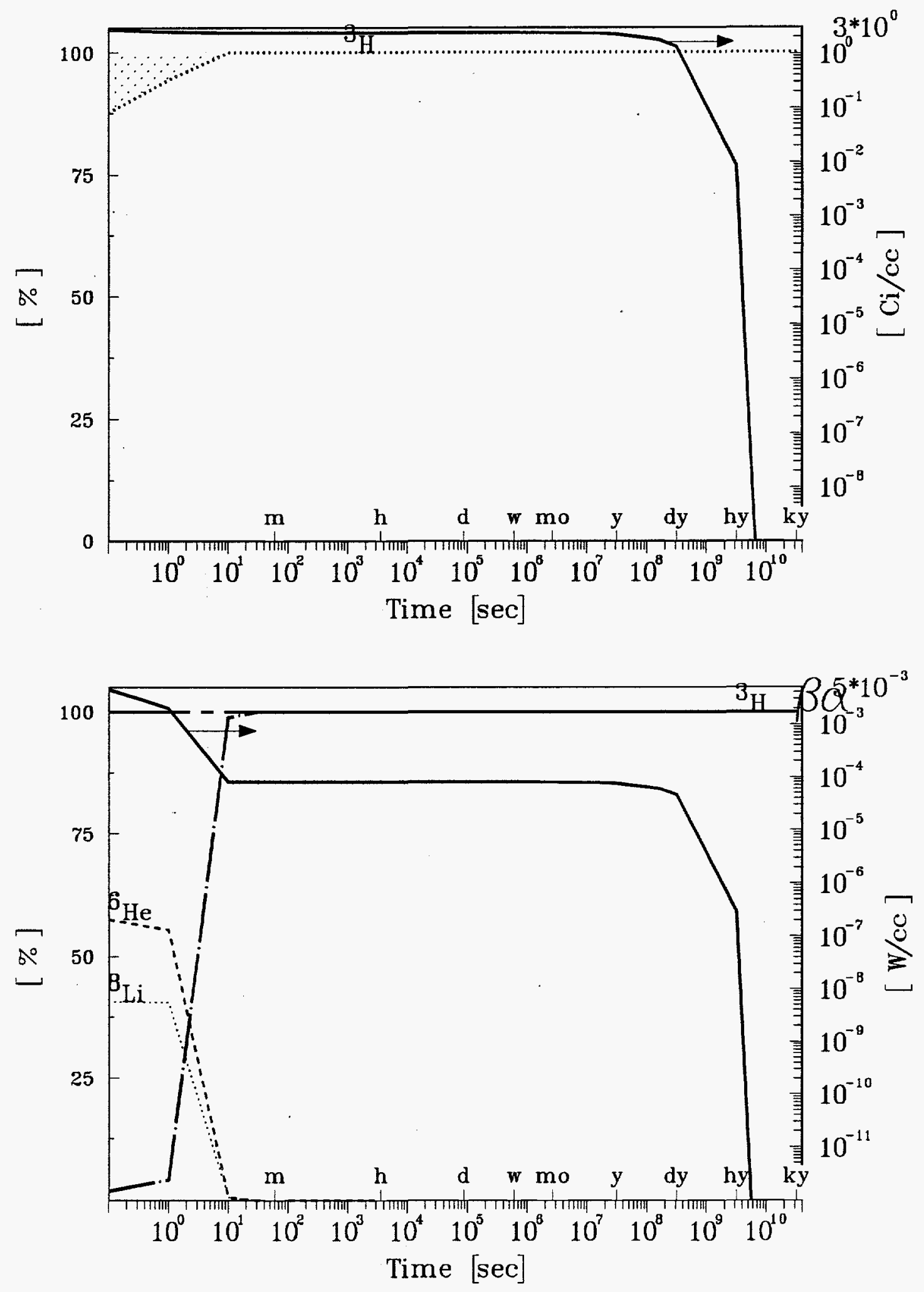

Figure 21. The specific radioactivity (top) and the specific decay heat (bottom) in zone \# 13 (I_Li - Li/V). 


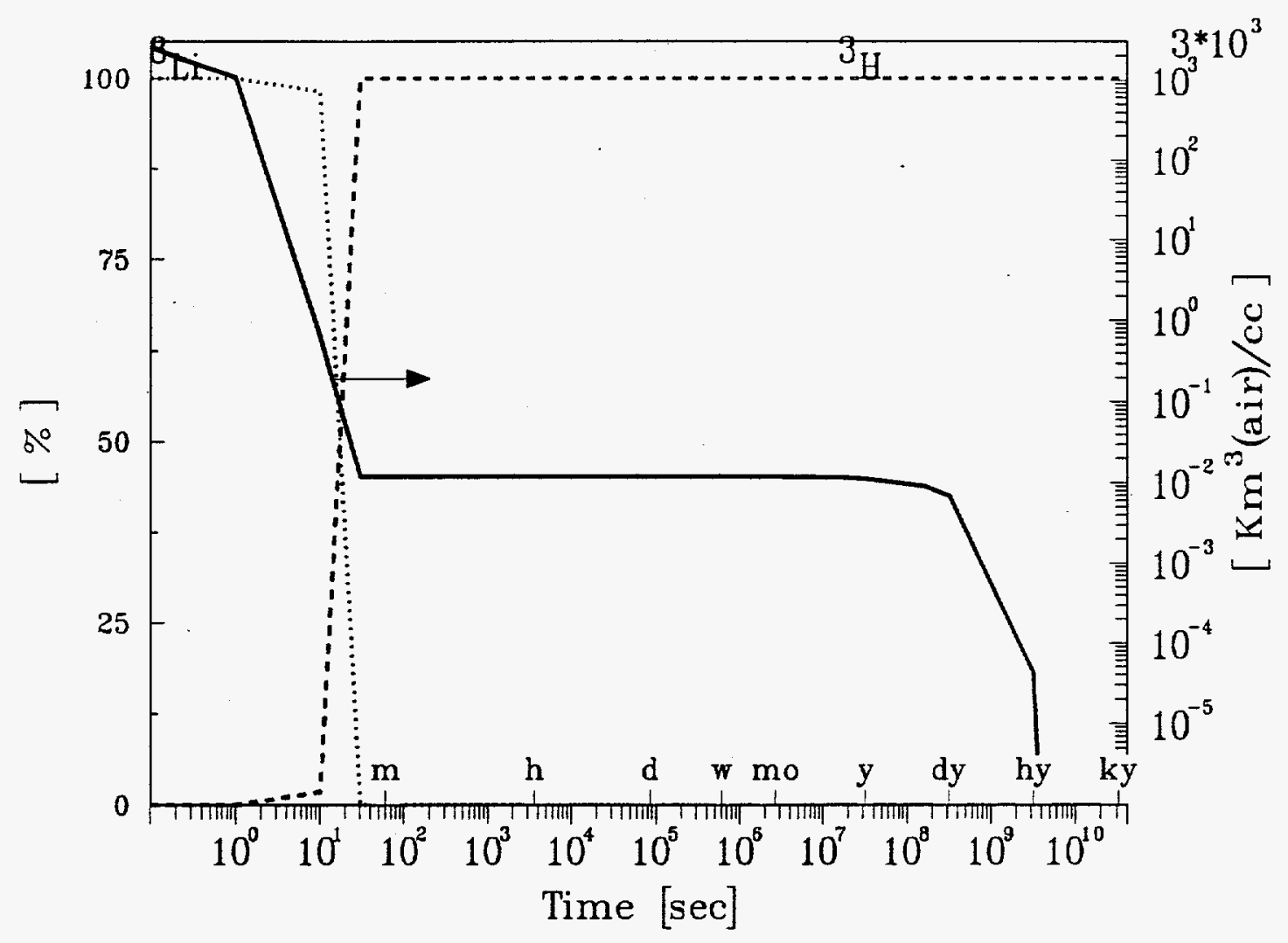

Figure 22. The specific air-BHP in zone \# $13\left(\mathrm{I} \_\mathrm{Li}-\mathrm{Li} / \mathrm{V}\right)$. 

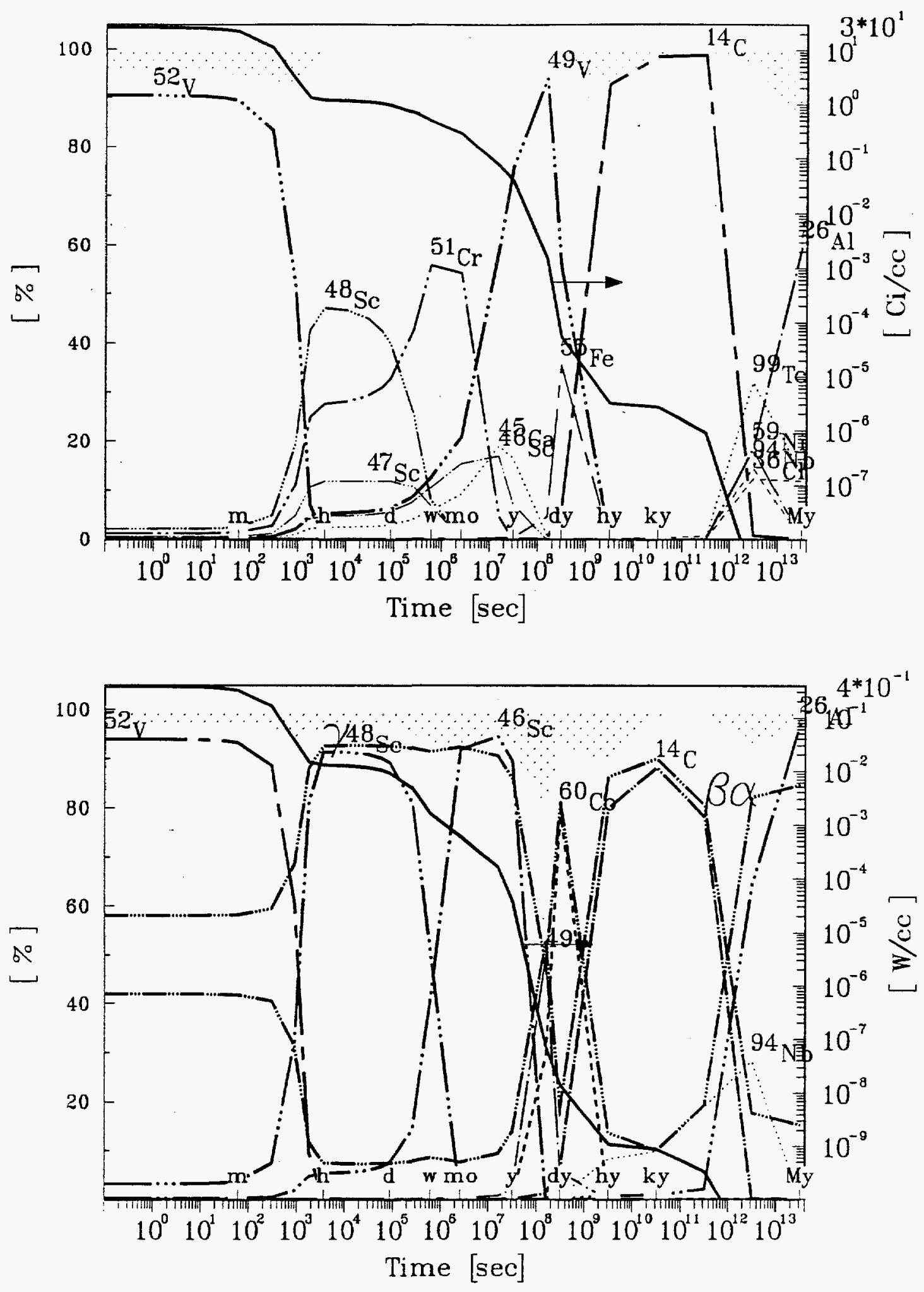

Figure 23. The specific radioactivity (top) and the specific decay heat (bottom) in zone \# 12 (I_Vall - Li/V). 

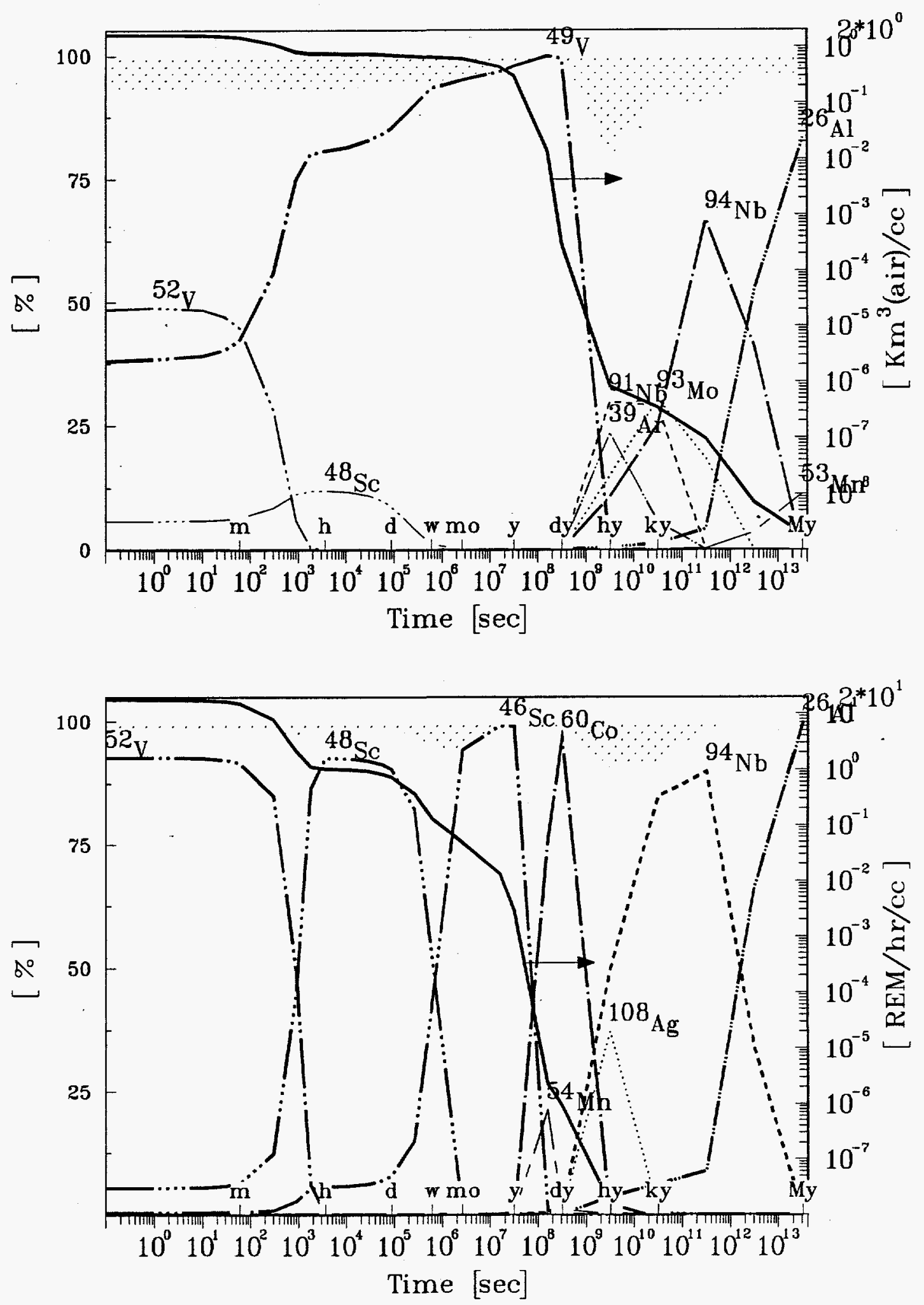

Figure 24. The specific air-BHP (top) and the point-source contact dose (bottom) in zone \# 12 (I_Vall - Li/V). 

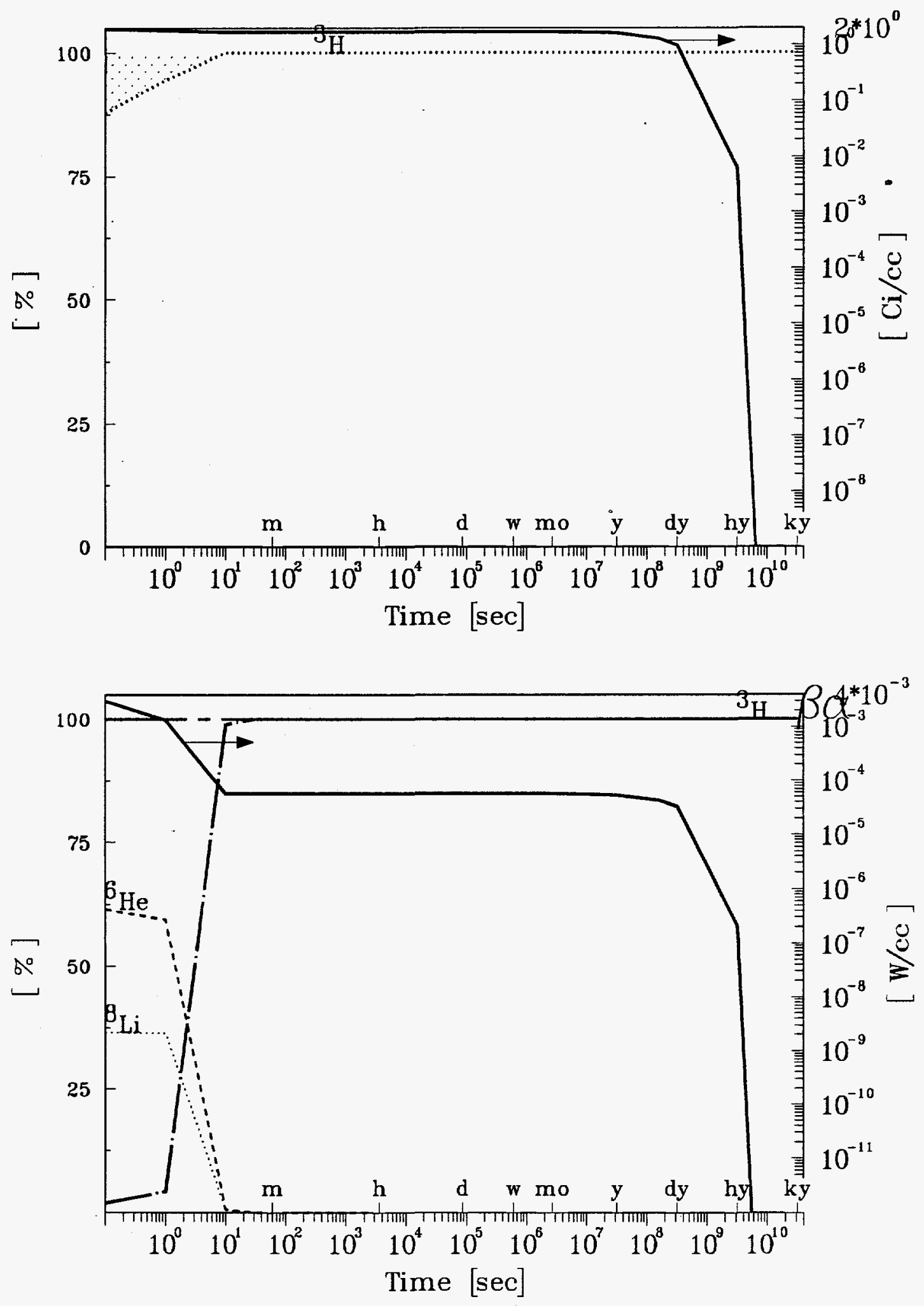

Figure 25. The specific radioactivity (top) and the specific decay heat (bottom) in zone \# $11\left(\mathrm{I} \_\mathrm{Li}-\mathrm{Li} / \mathrm{V}\right)$. 


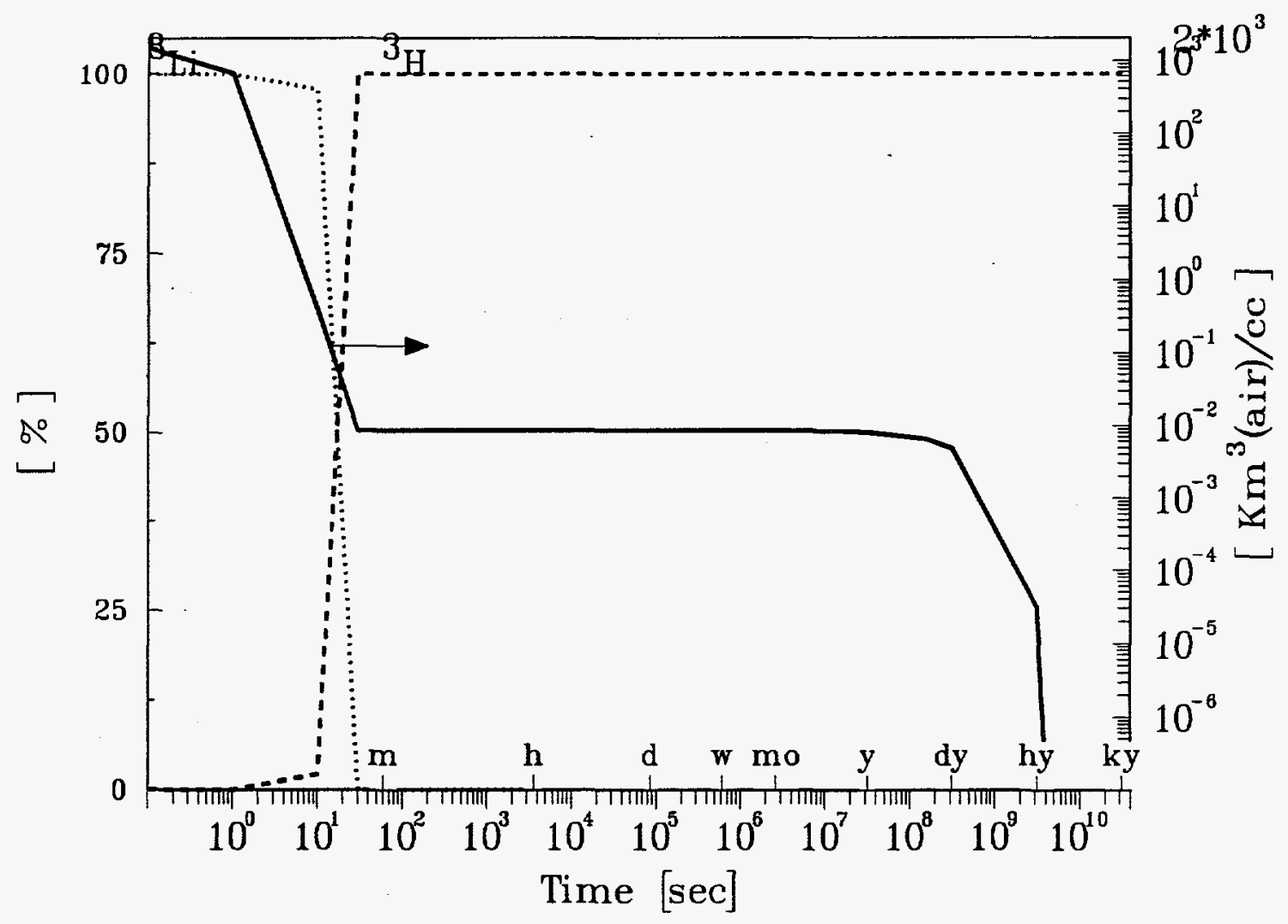

Figure 26. The specific air-BHP in zone \#11 (I_Li - Li/V). 

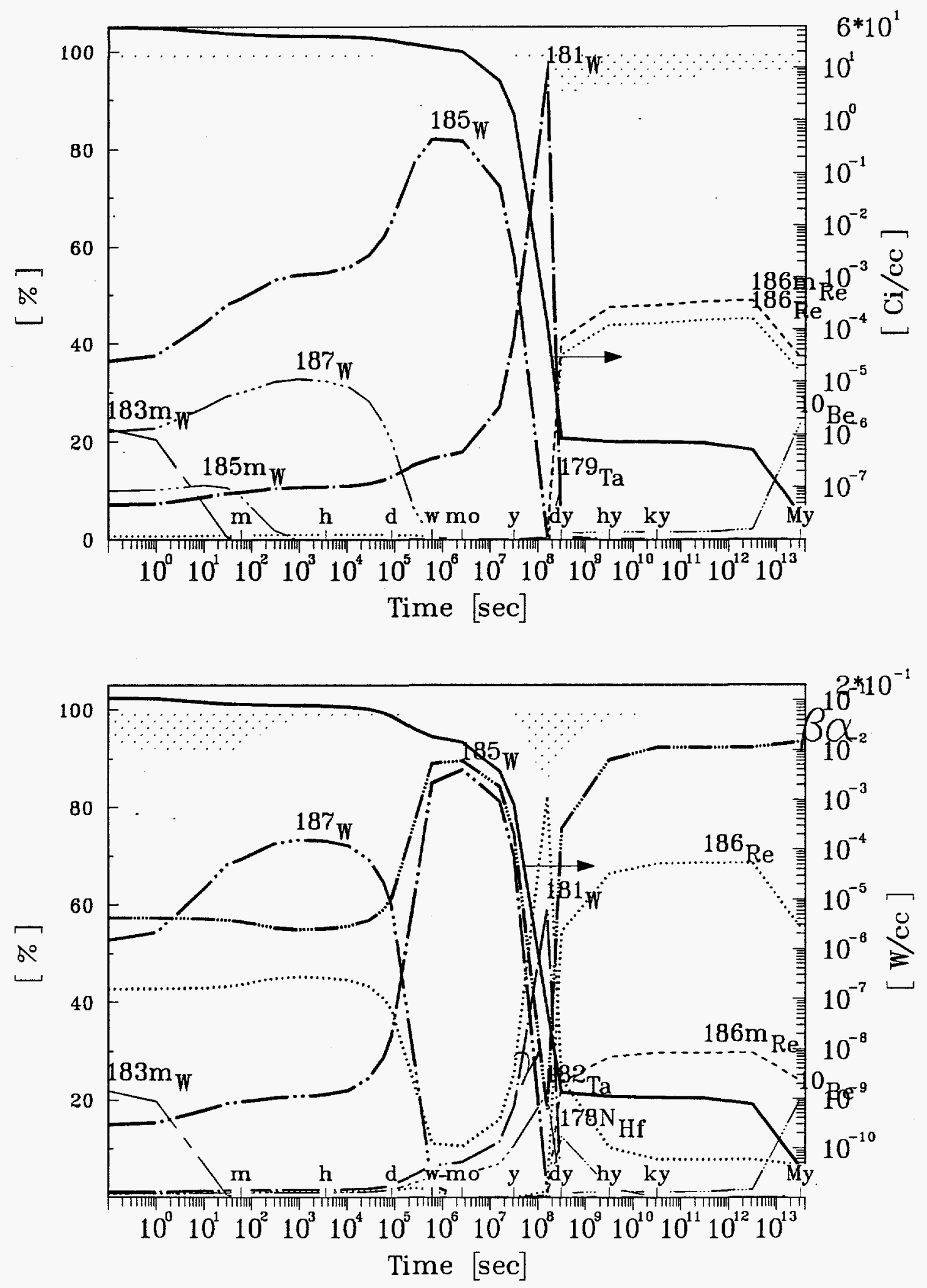

10

Figure 27 . The specific radioactivity (top) and the specific decay heat (bottom) in zone \# 10 (I_WC - Li/V). 

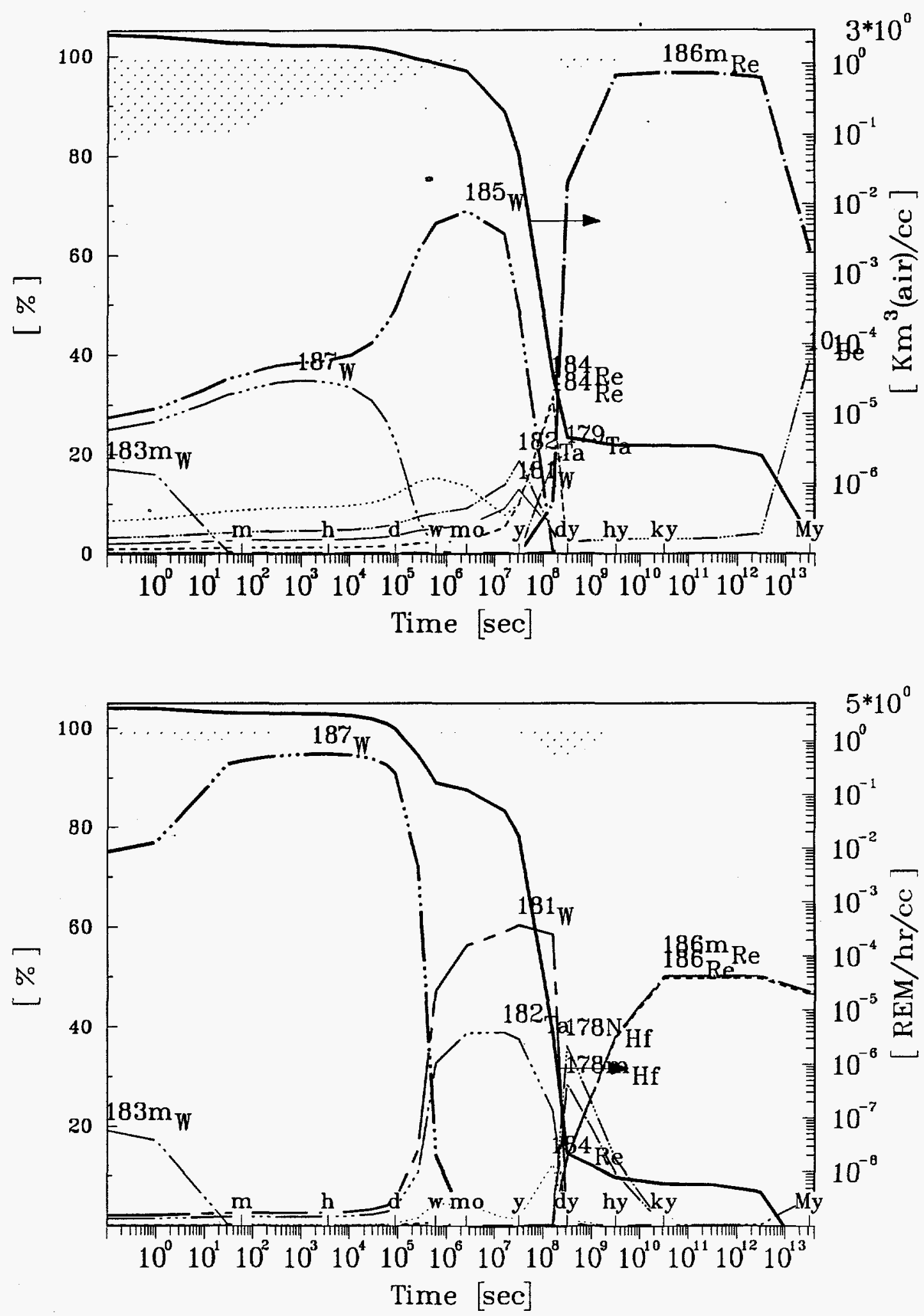

Figure 28. The specific air-BHP (top) and the point-source contact dose (bottom) in zone \# 10 (I_WC - Li/V). 

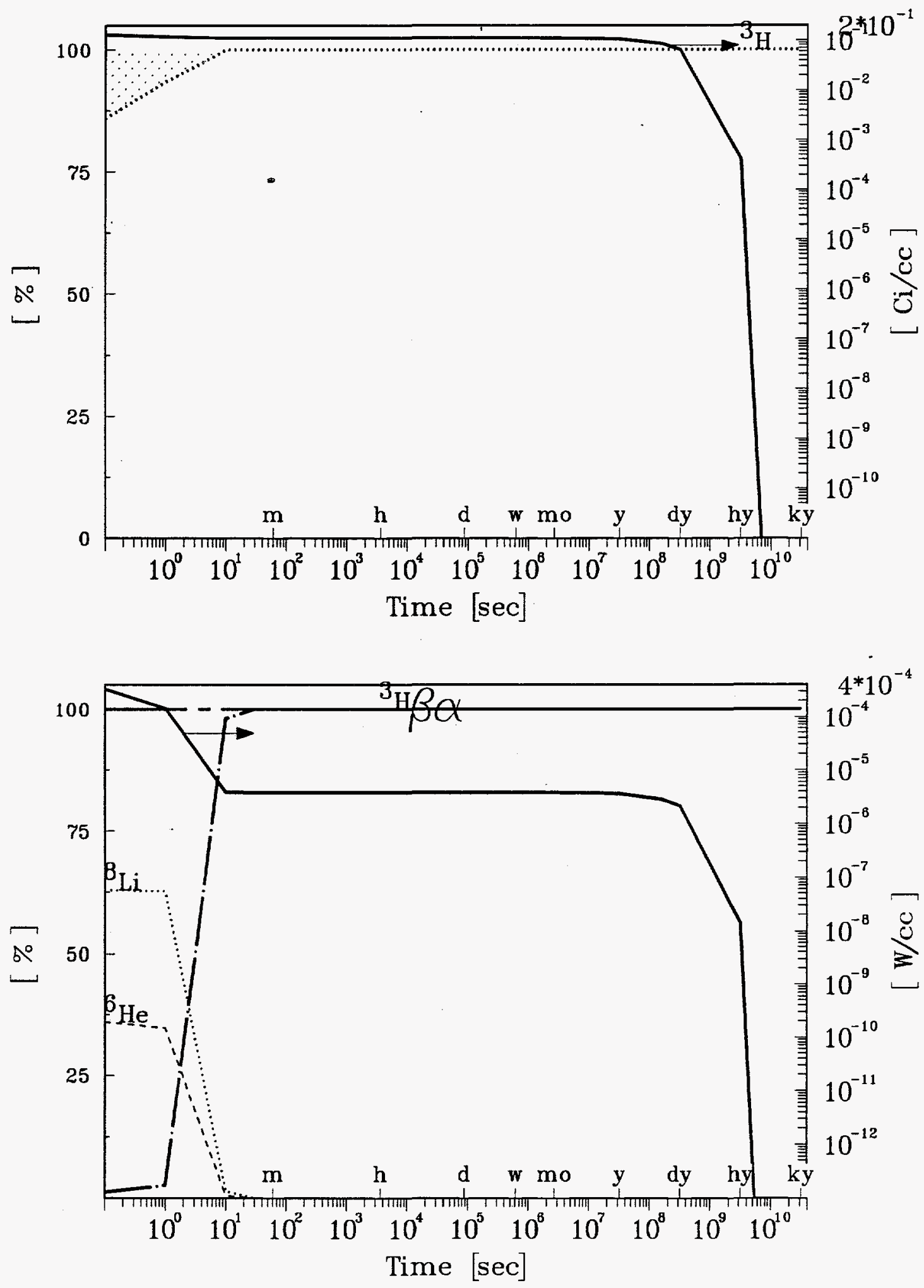

Figure 29. The specific radioactivity (top) and the specific decay heat (bottom) in zone \# $9\left(\mathrm{I} \_\mathrm{Li}-\mathrm{Li} / \mathrm{V}\right)$. 


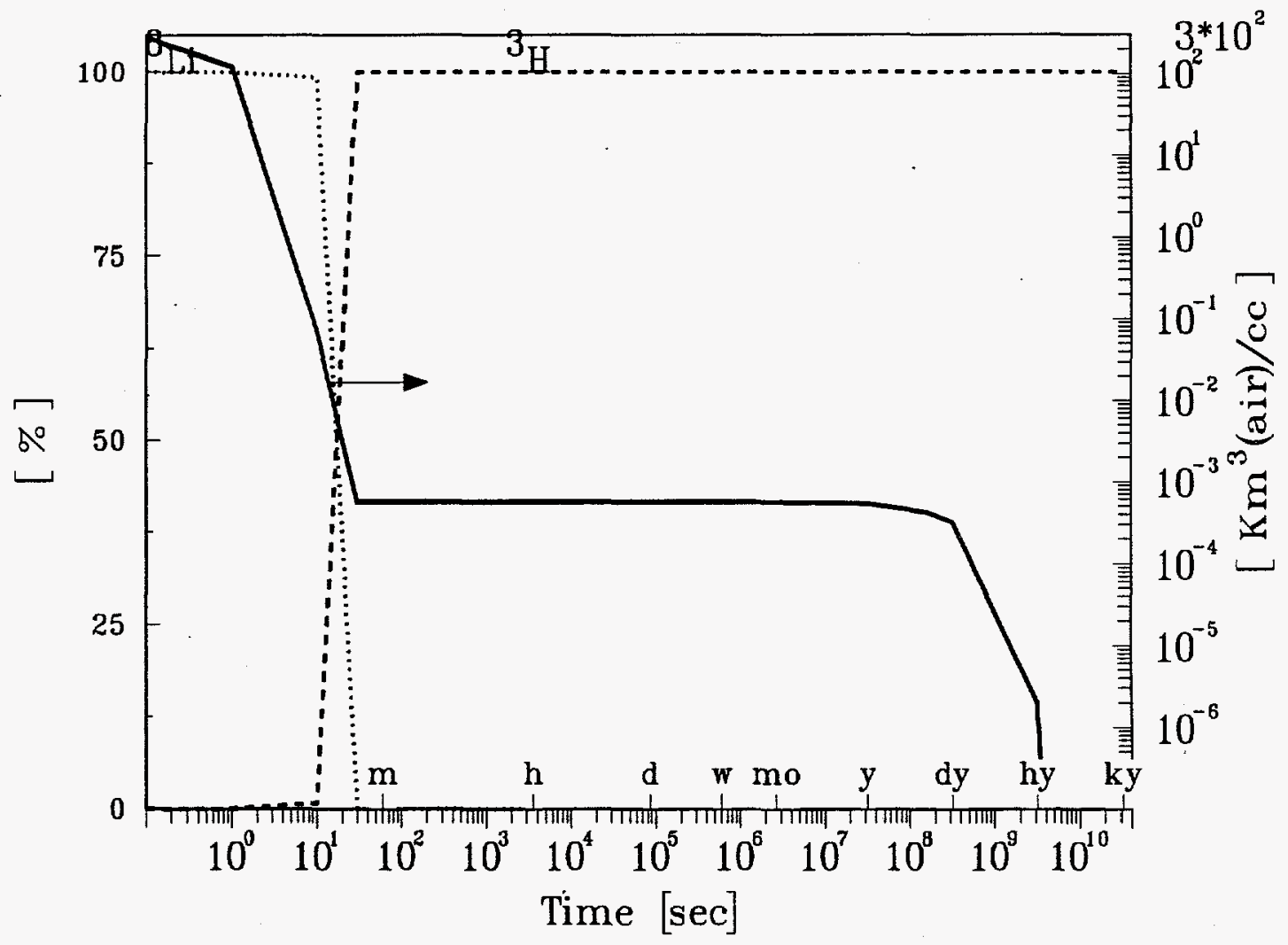

Figure 30. The specific air-BHP in zone \# $9\left(\mathrm{I} \_\mathrm{Li}-\mathrm{Li} / \mathrm{V}\right)$. 

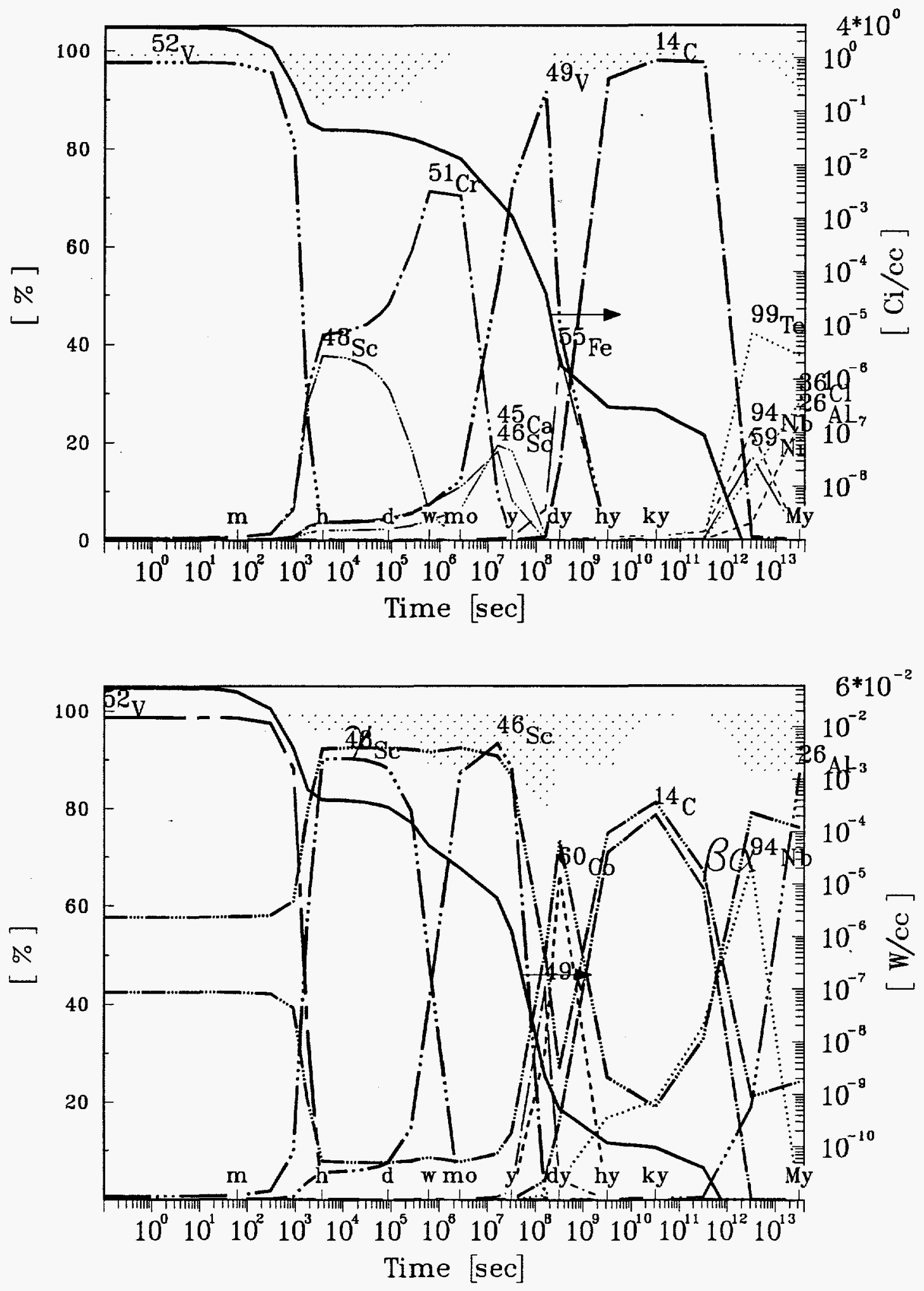

Figure 31. The specific radioactivity (top) and the specific decay heat (bottom) in zone \# 8 (I_Vall - Li/V). 

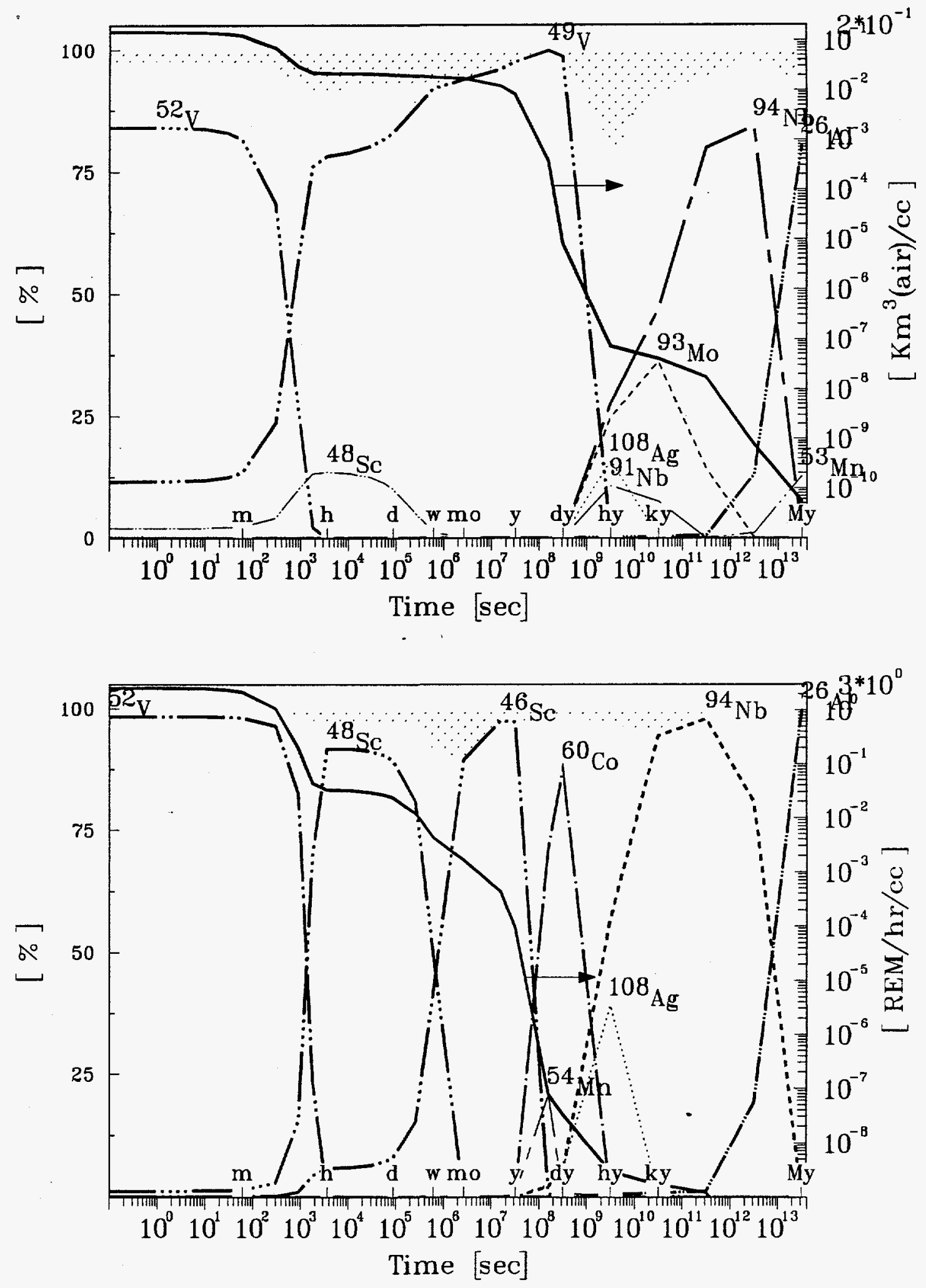

Figure 32. The specific air-BHP (top) and the point-source contact dose (bottom) in zone \# 8 (I_Vall - Li/V). 

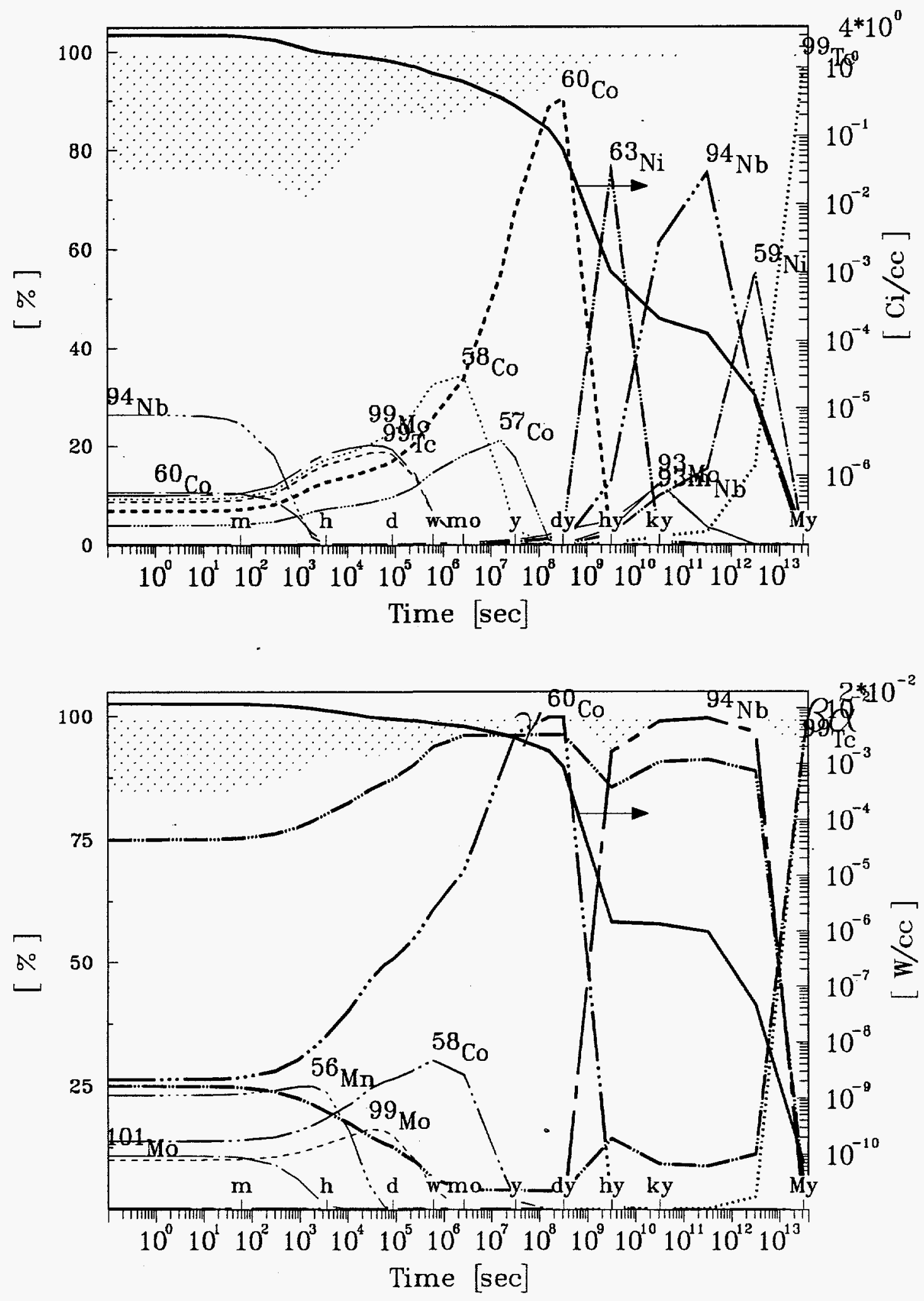

Figure 33. The specific radioactivity (top) and the specific decay heat (bottom) in zone \# 7 (I_Incon - Li/V). 

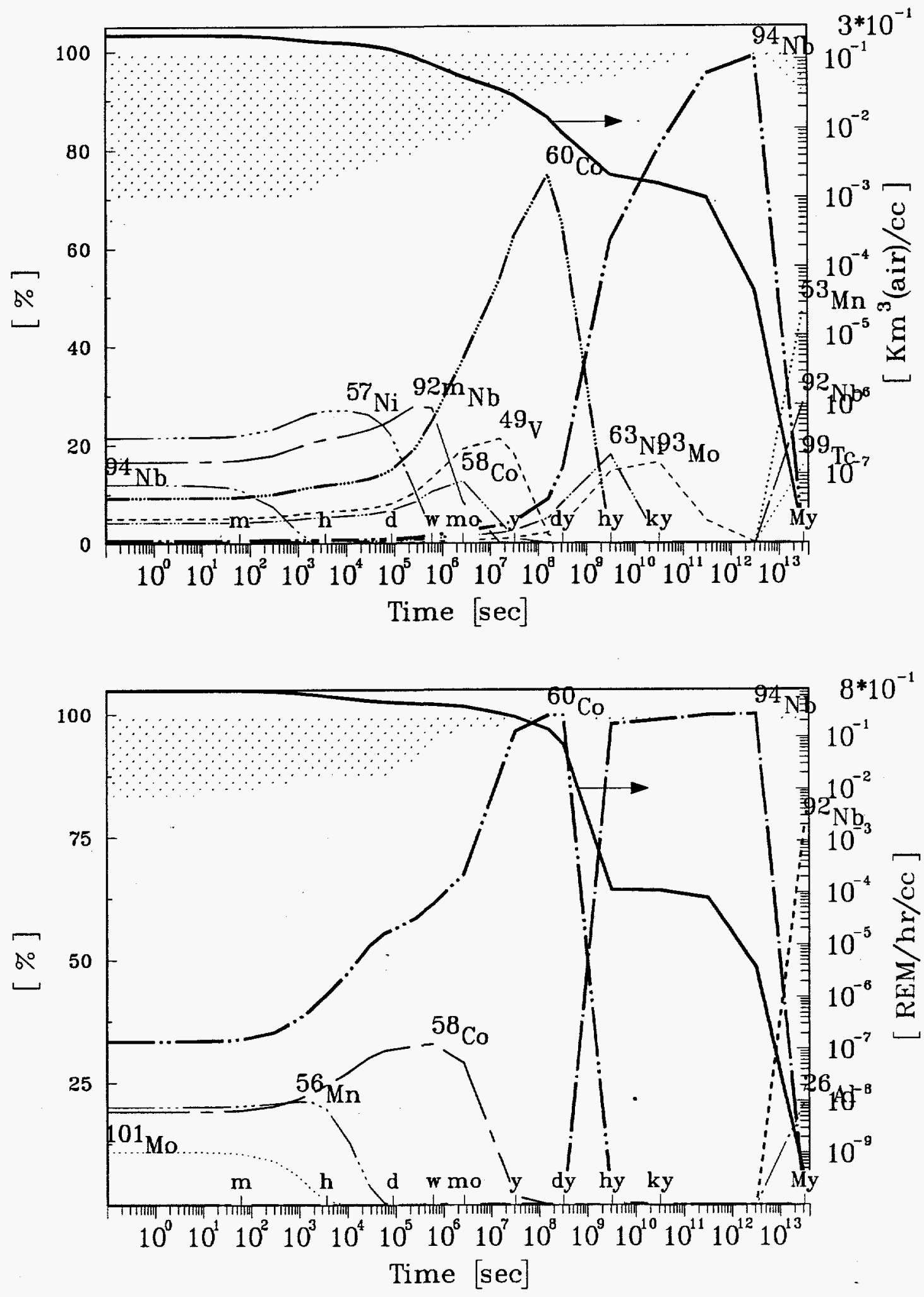

Figure 34. The specific air-BHP (top) and the point-source contact dose (bottom) in zone \# 7 (I_Incon - Li/V). 

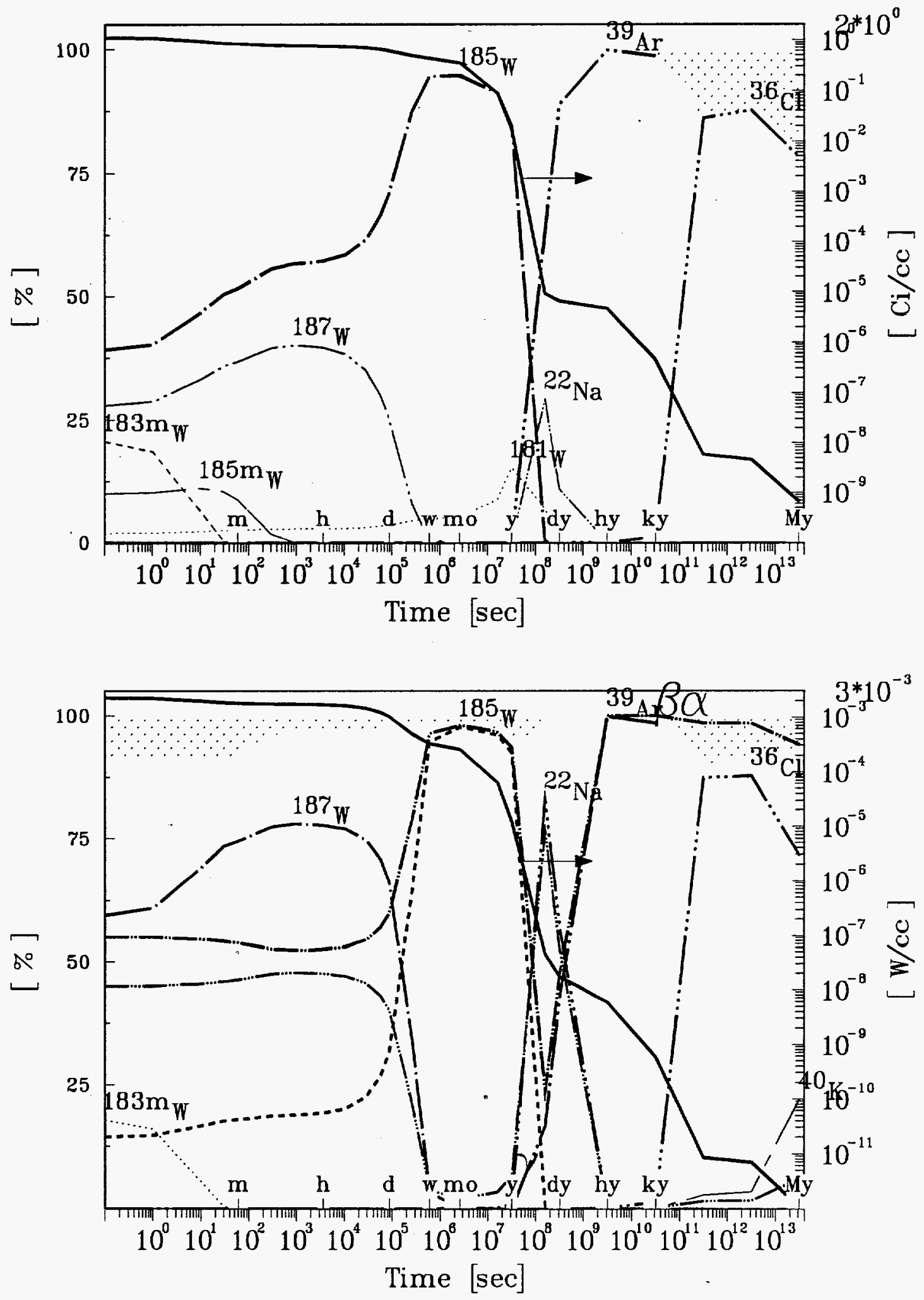

Figure 35. The specific radioactivity (top) and the specific decay heat (bottom) in zone \# 6 (I_WNaK - Li/V). 

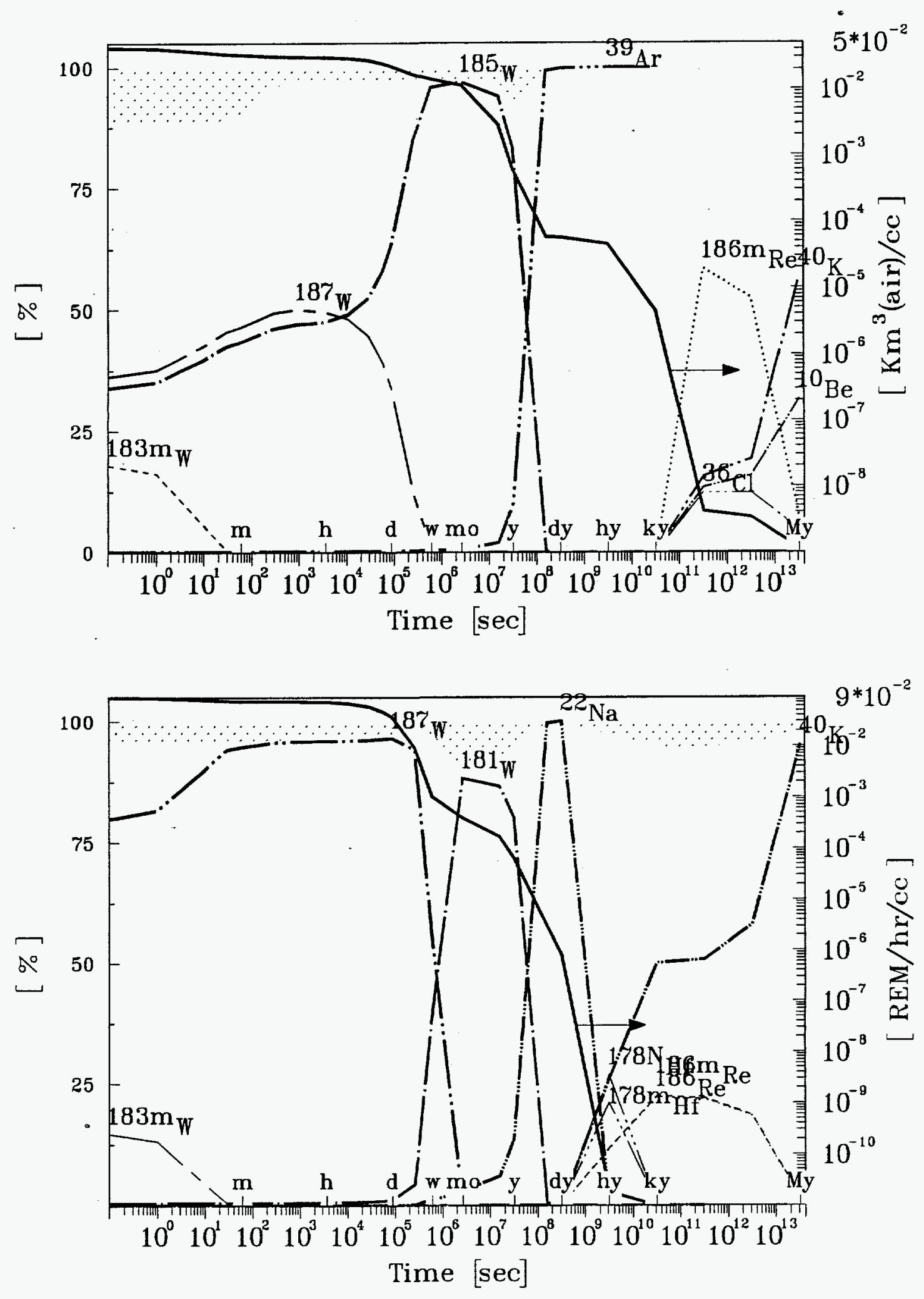

Figure 36. The specific air-BHP (top) and the point-source contact dose (bottom) in zone \# 6 (I_WNaK - Li/V). 

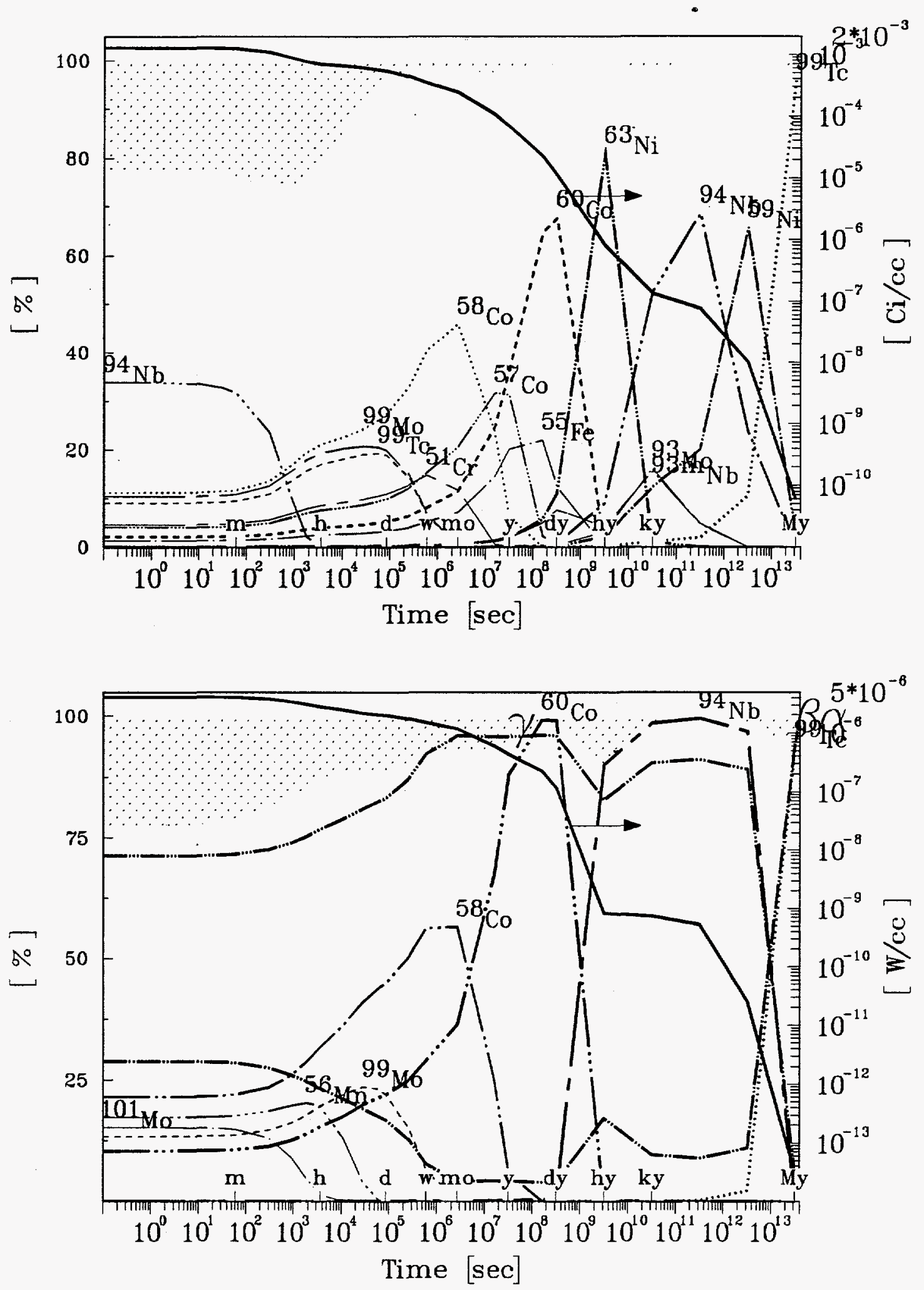

Figure 37. The specific radioactivity (top) and the specific decay heat (bottom) in zone \# 5 (I_Incon - Li/V). 

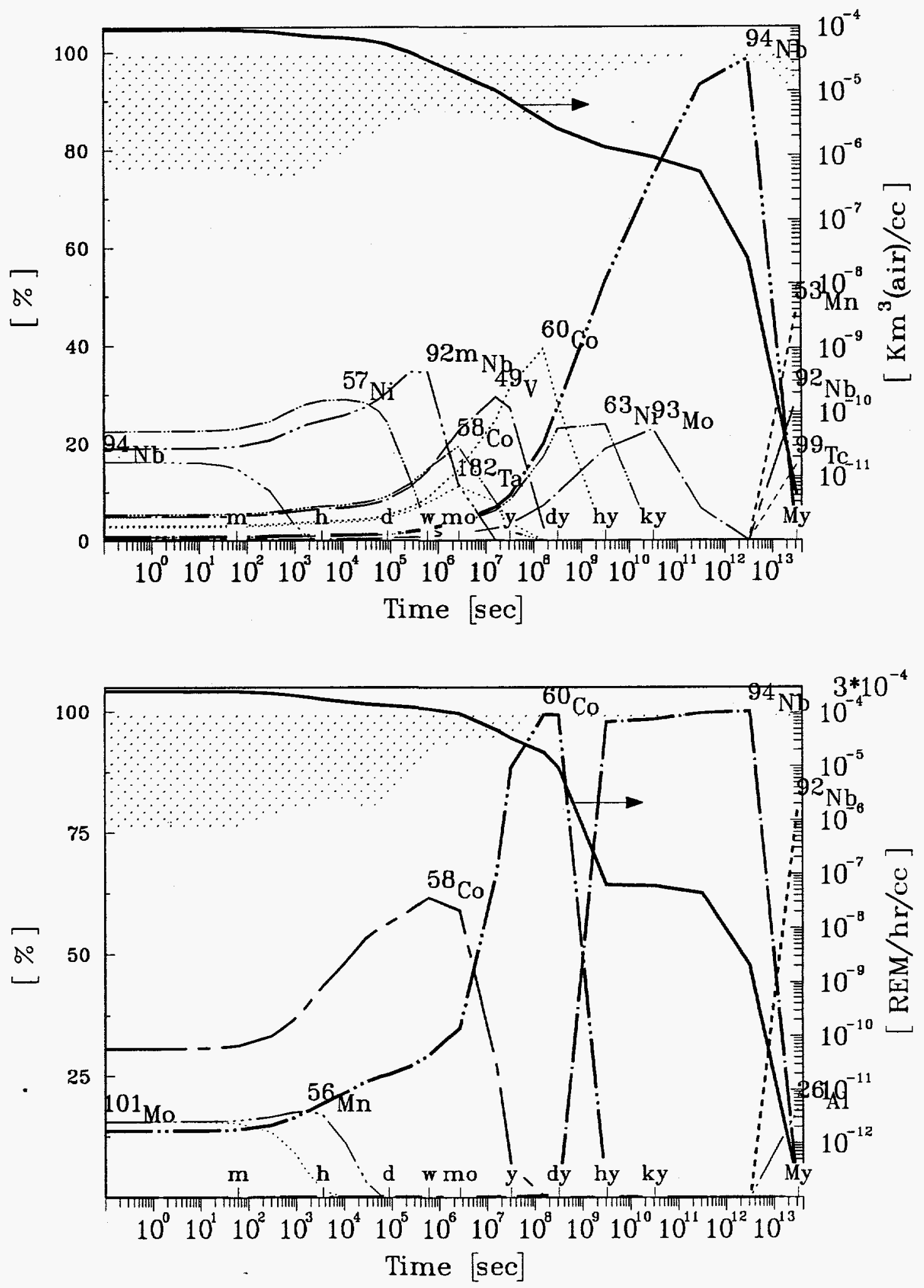

Figure 38. The specific air-BHP (top) and the point-source contact dose (bottom) in zone \# 5 (I_Incon - Li/V). 

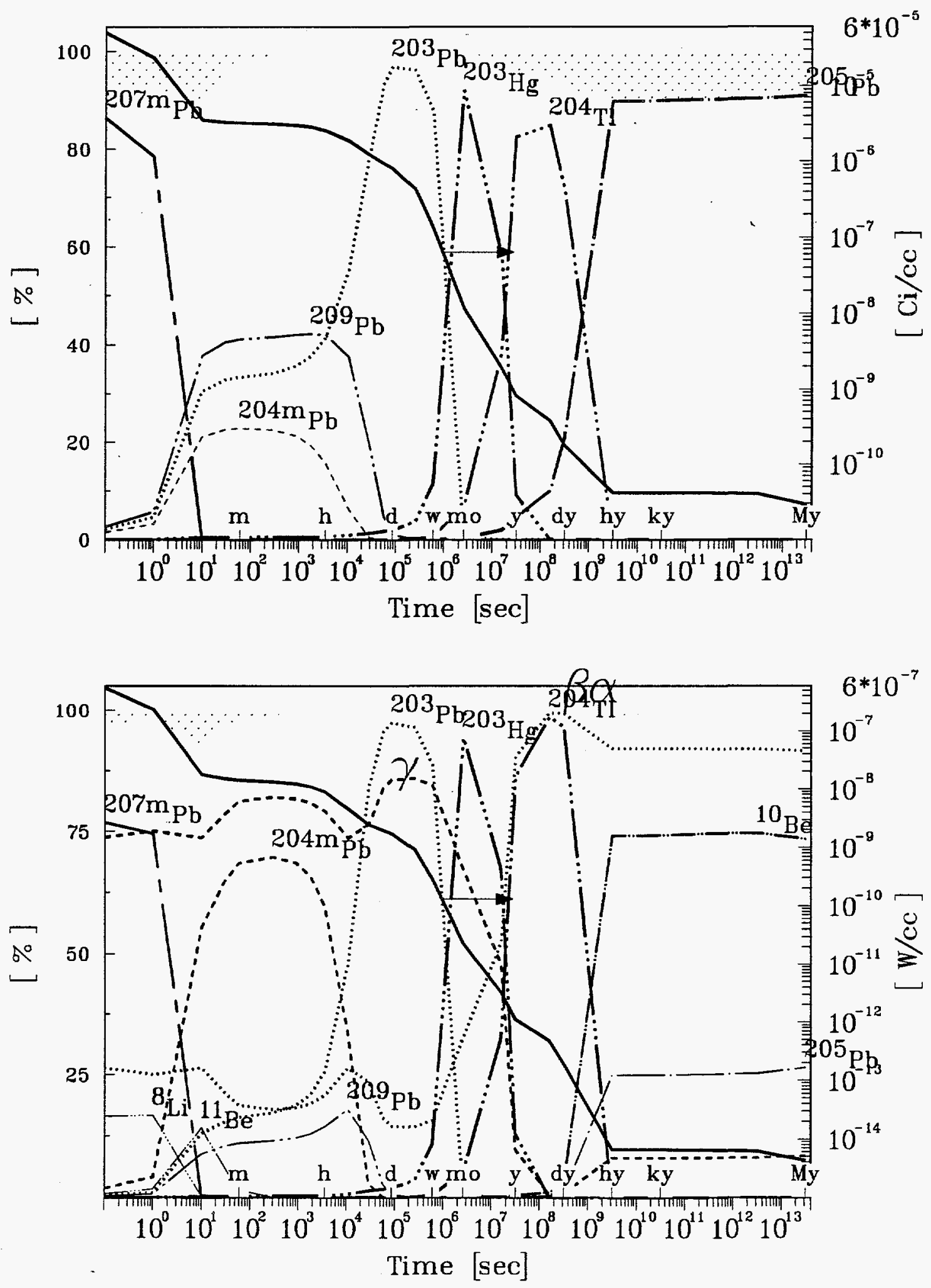

Figure 39. The specific radioactivity (top) and the specific decay heat (bottom) in zone \# 4 (I_B4C_Pb - Li/V). 

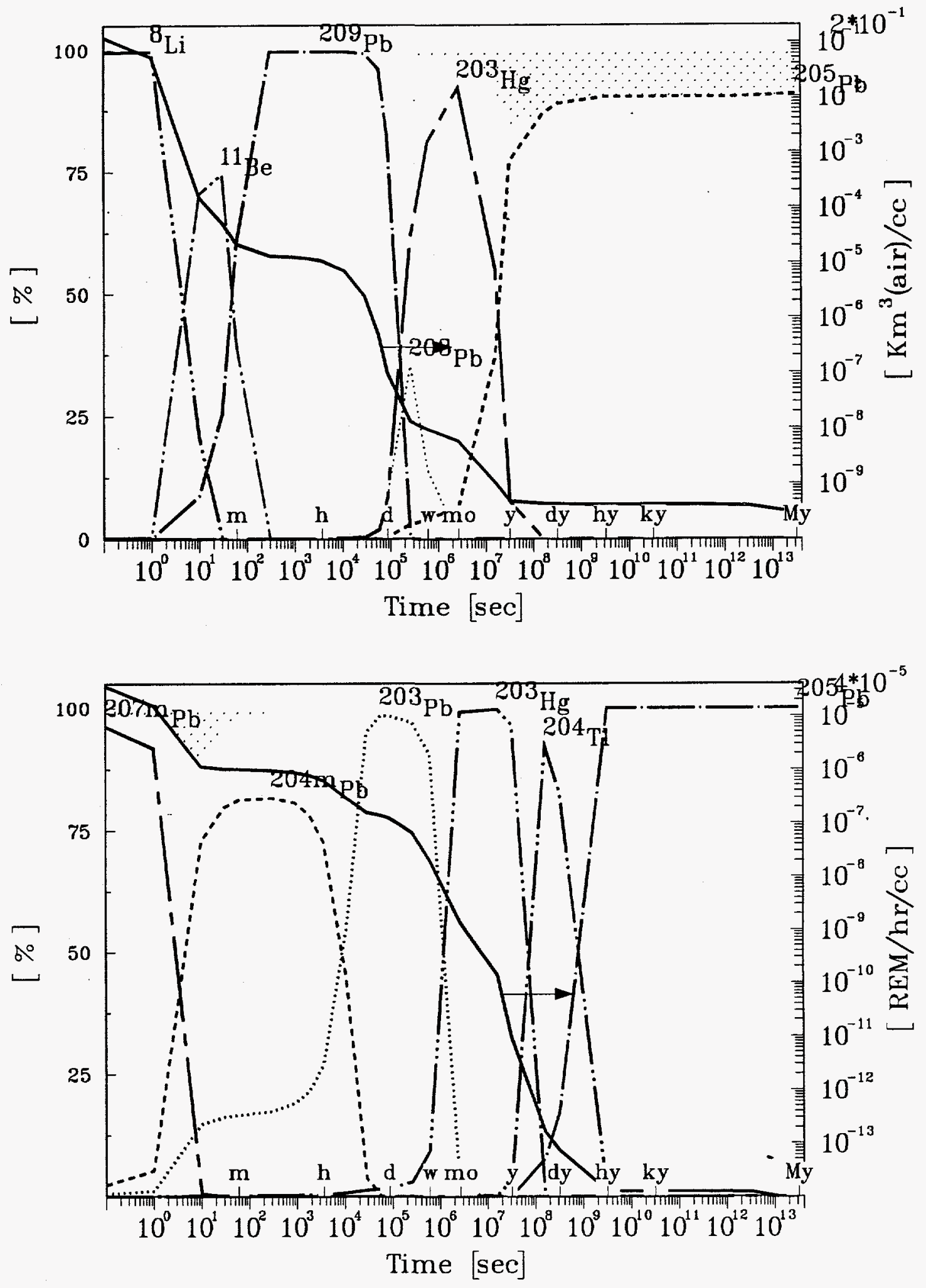

Figure 40. The specific air-BHP (top) and the point-source contact dose (bottom) in zone \# 4 (I_B4C_Pb - Li/V). 

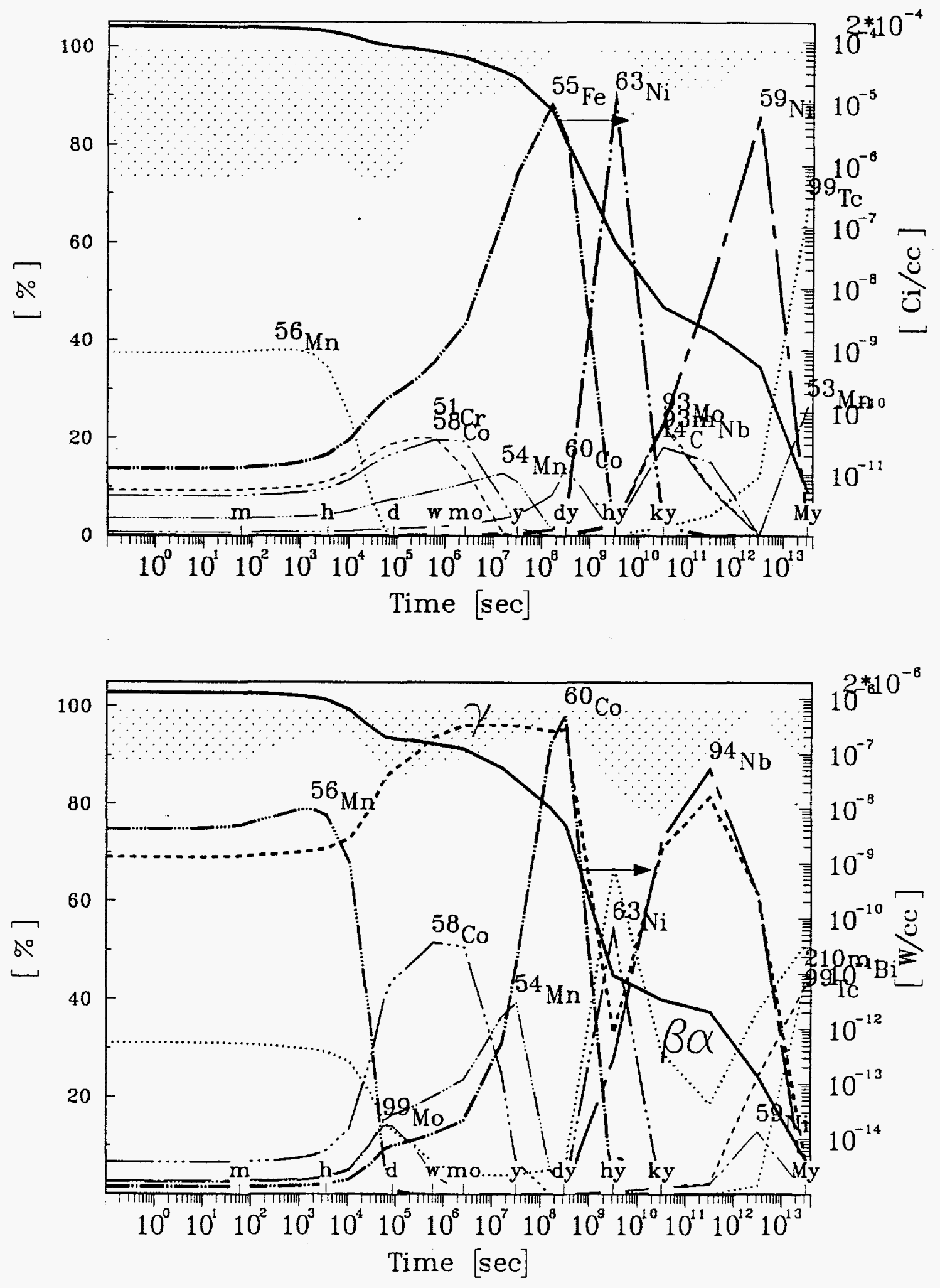

Figure 41 . The specific radioactivity (top) and the specific decay heat (bottom) in zone \# 3 (I_SSWP - Li/V). 

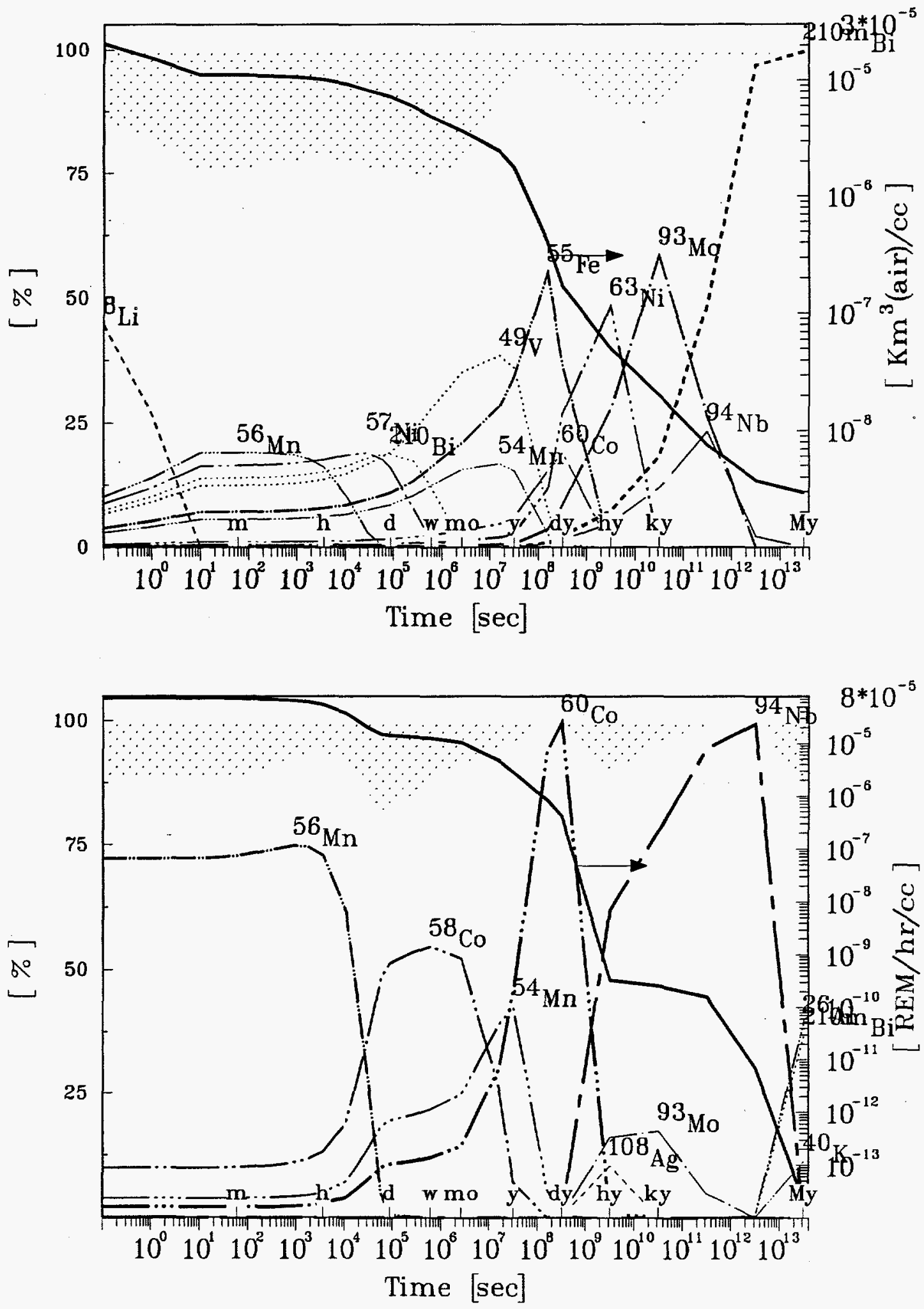

Figure 42. The specific air-BHP (top) and the point-source contact dose (bottom) in zone \# 3 (I_SSWP - Li/V). 

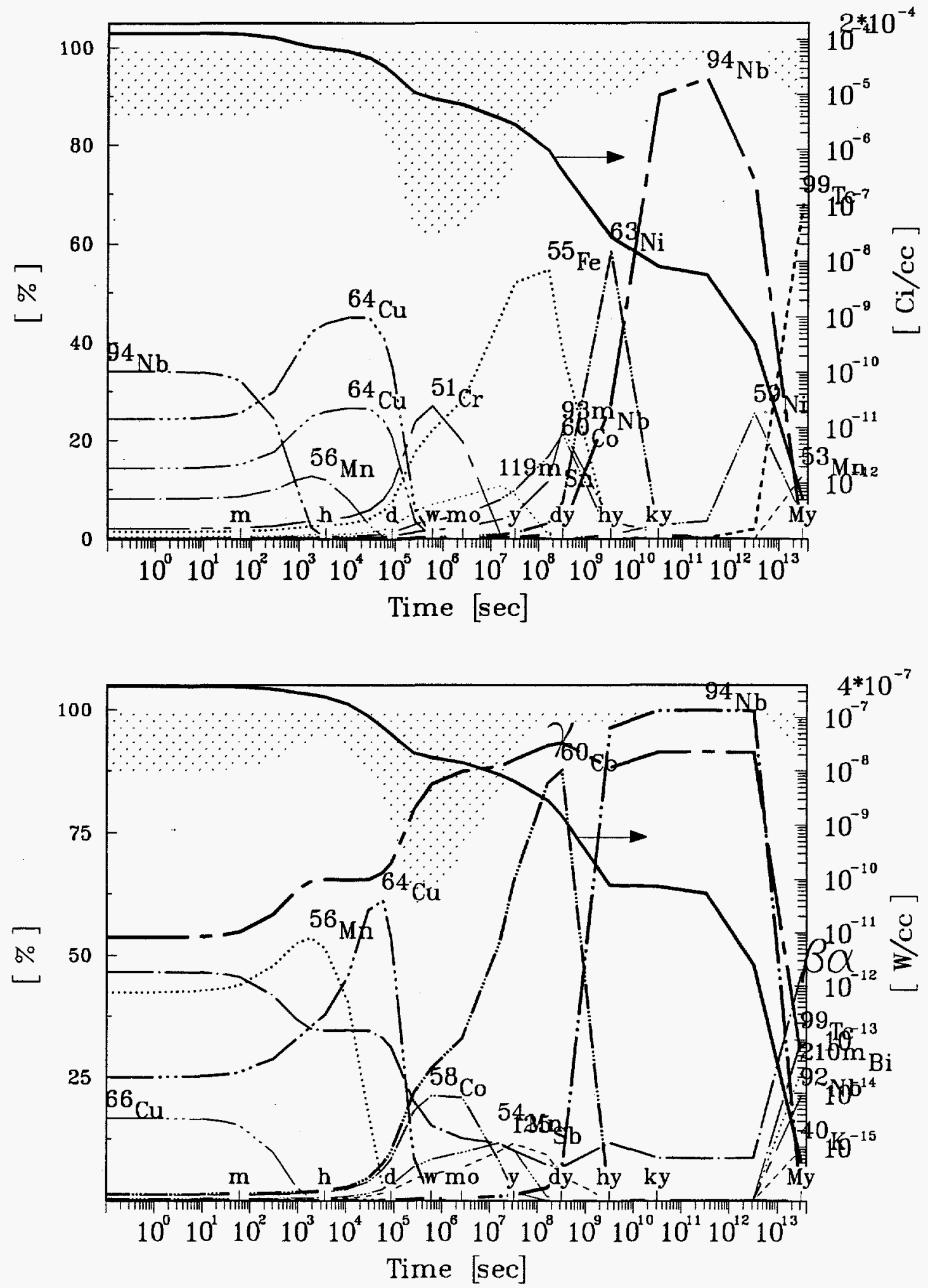

Figure 43. The specific radioactivity (top) and the specific decay heat (bottom) in zone \# $2($ I_coil - Li/V). 

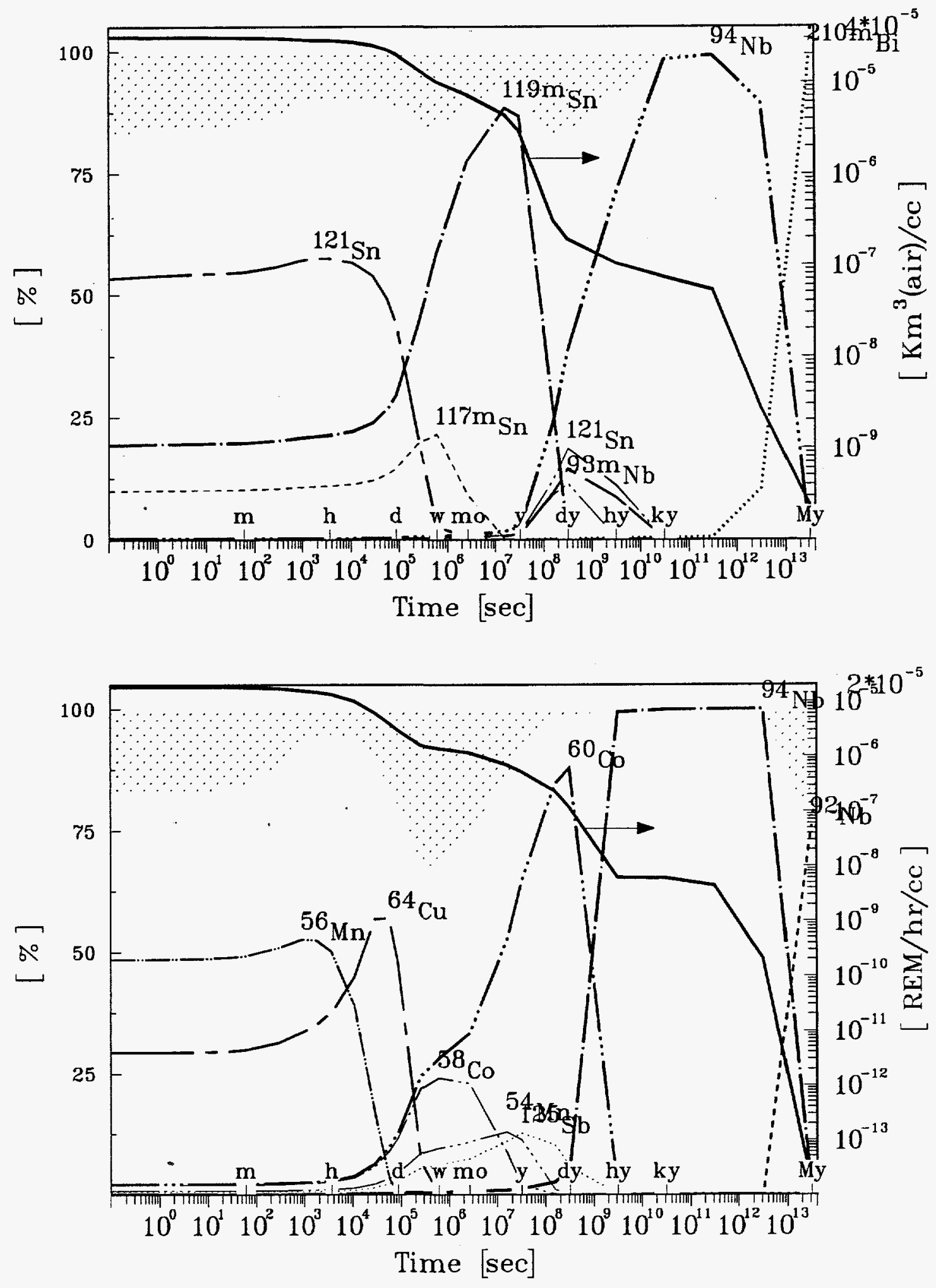

Figure 44. The specific air-BHP (top) and the point-source contact dose (bottom) in zone \# 2 (I_coil - Li/V). 

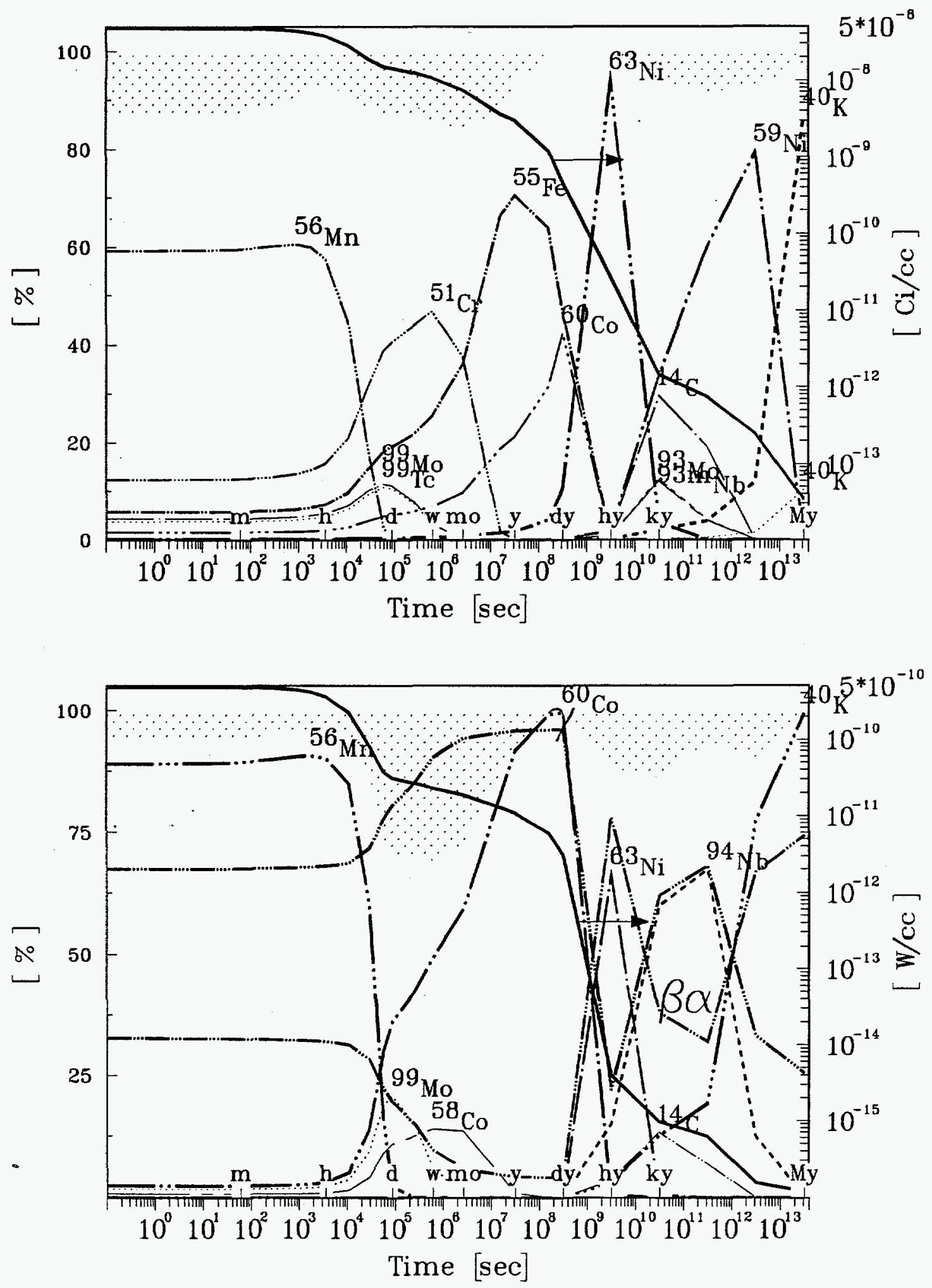

Figure 45. The specific radioactivity (top) and the specific decay heat (bottom) in zone \# 1 (I_SSWP - Li/V). 

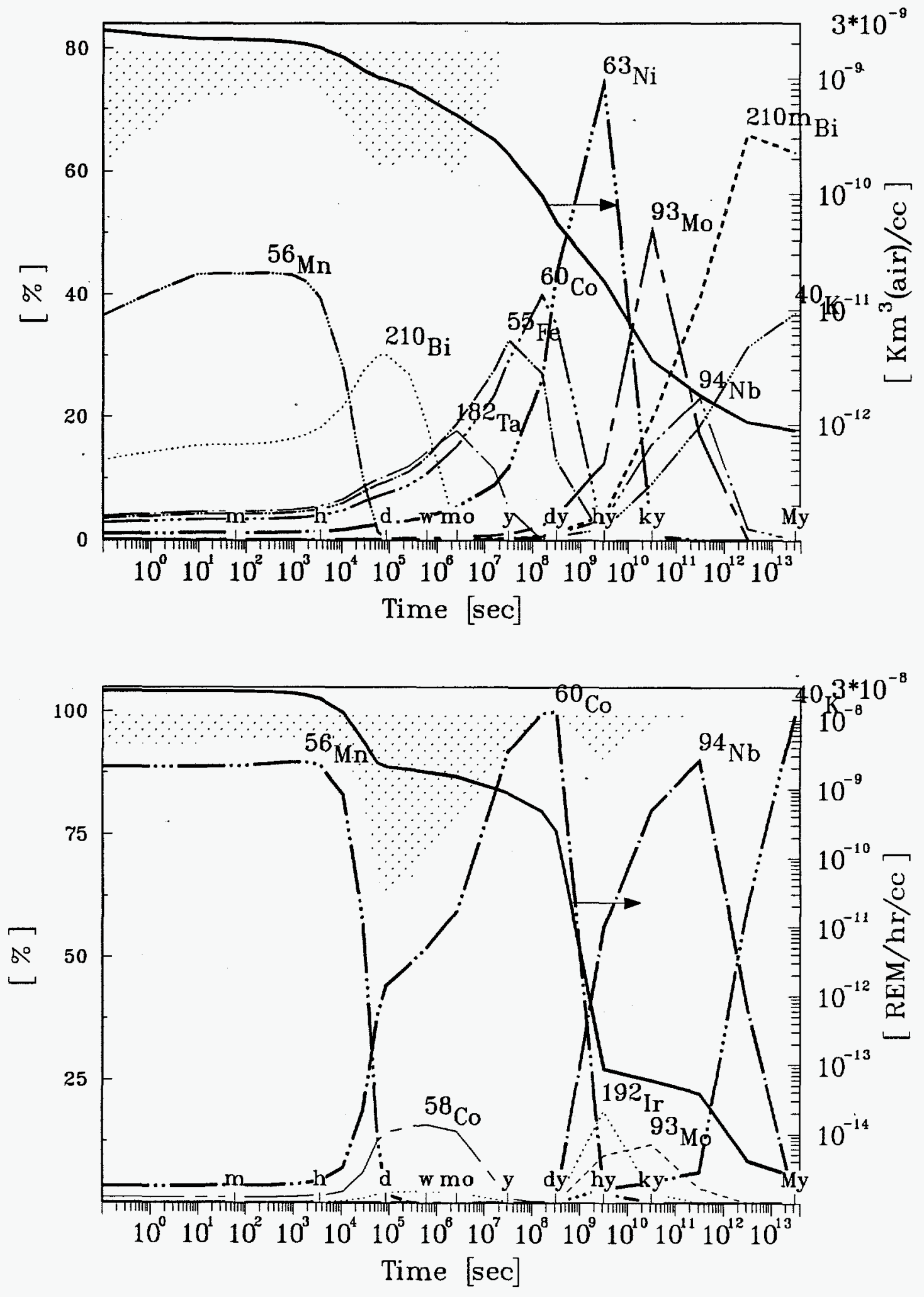

Figure 46. The specific air-BHP (top) and the point-source contact dose (bottom) in zone \# 1 (I_SSWP - Li/V). 

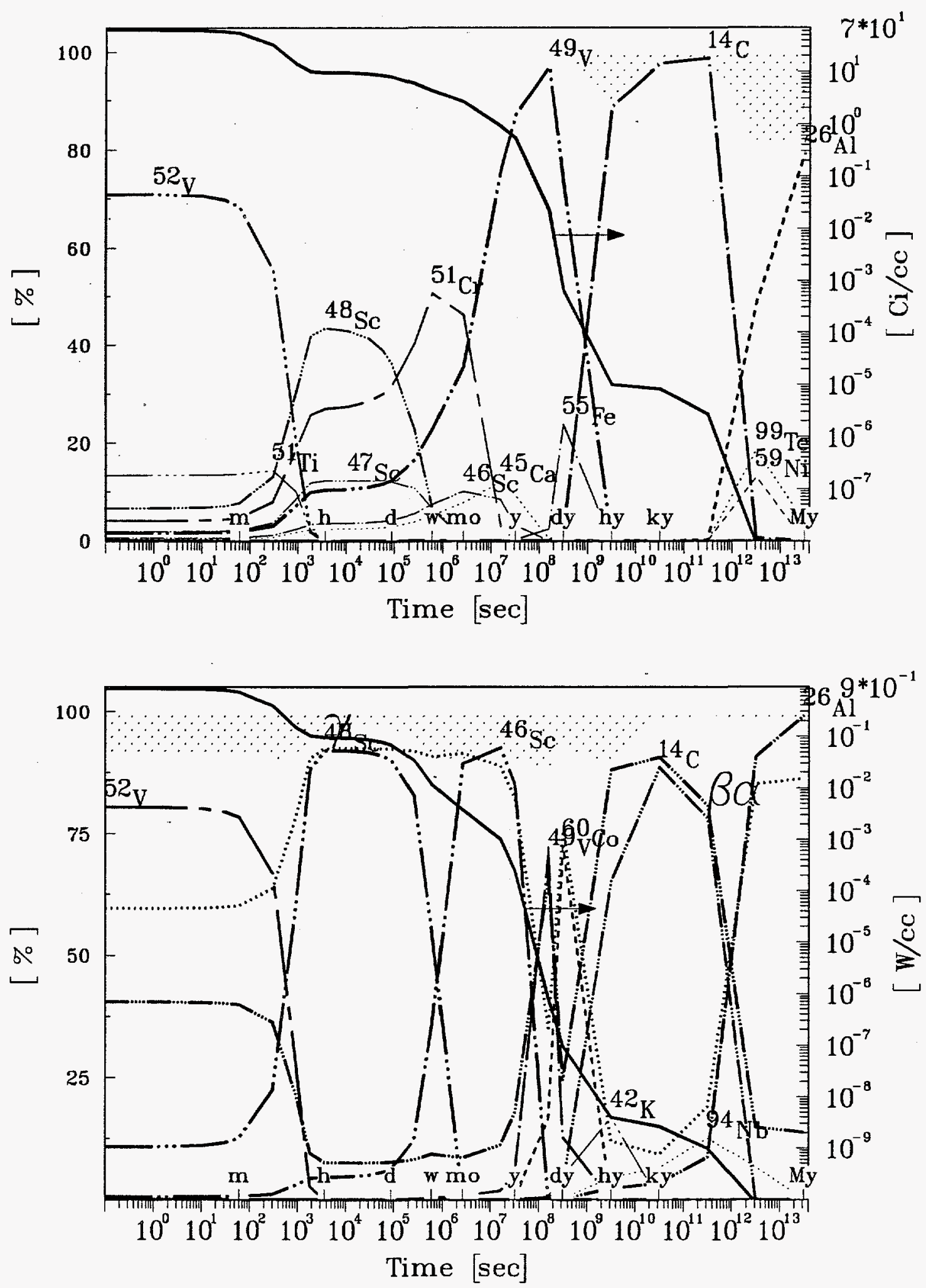

17

Figure 47. The specific radioactivity (top) and the specific decay heat (bottom) in zone \# 17 (O_Vall - Li/V). 

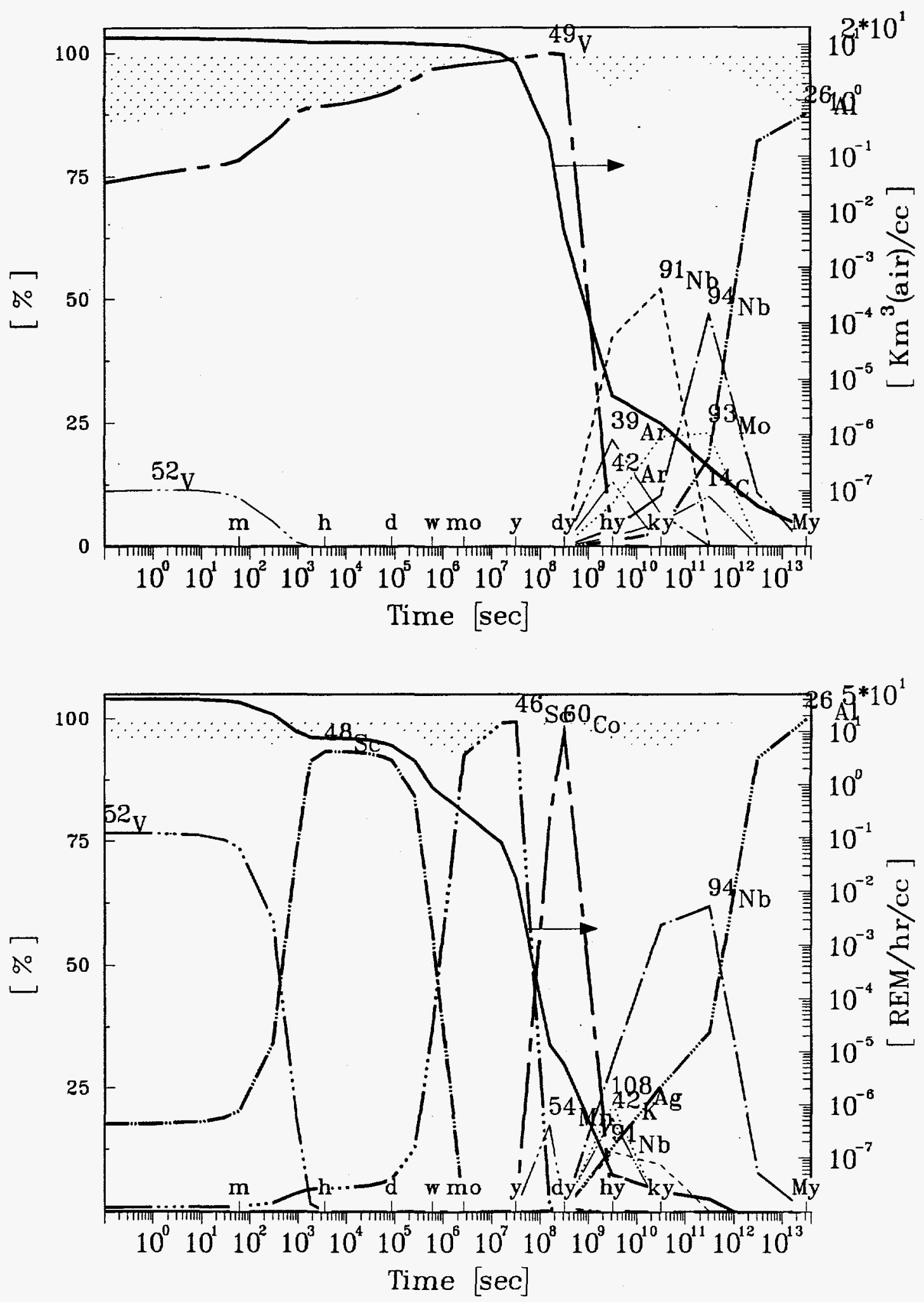

Figure 48. The specific air-BHP (top) and the point-source contact dose (bottom) in zone \# 17 (O_Vall - Li/V). 

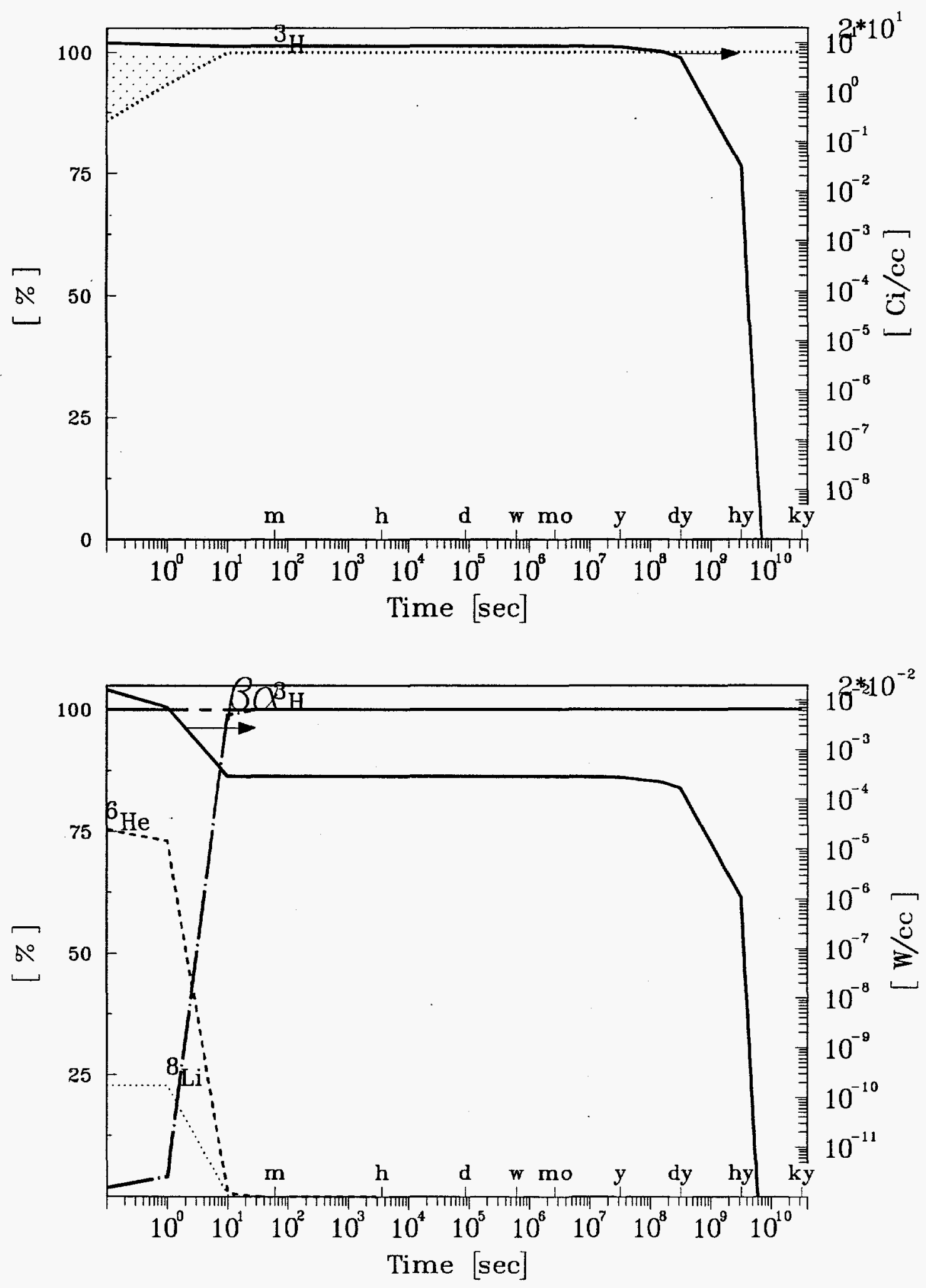

Figure 49. The specific radioactivity (top) and the specific decay heat (bottom) in zone \# $18\left(\mathrm{O} \_\mathrm{Li}-\mathrm{Li} / \mathrm{V}\right)$. 


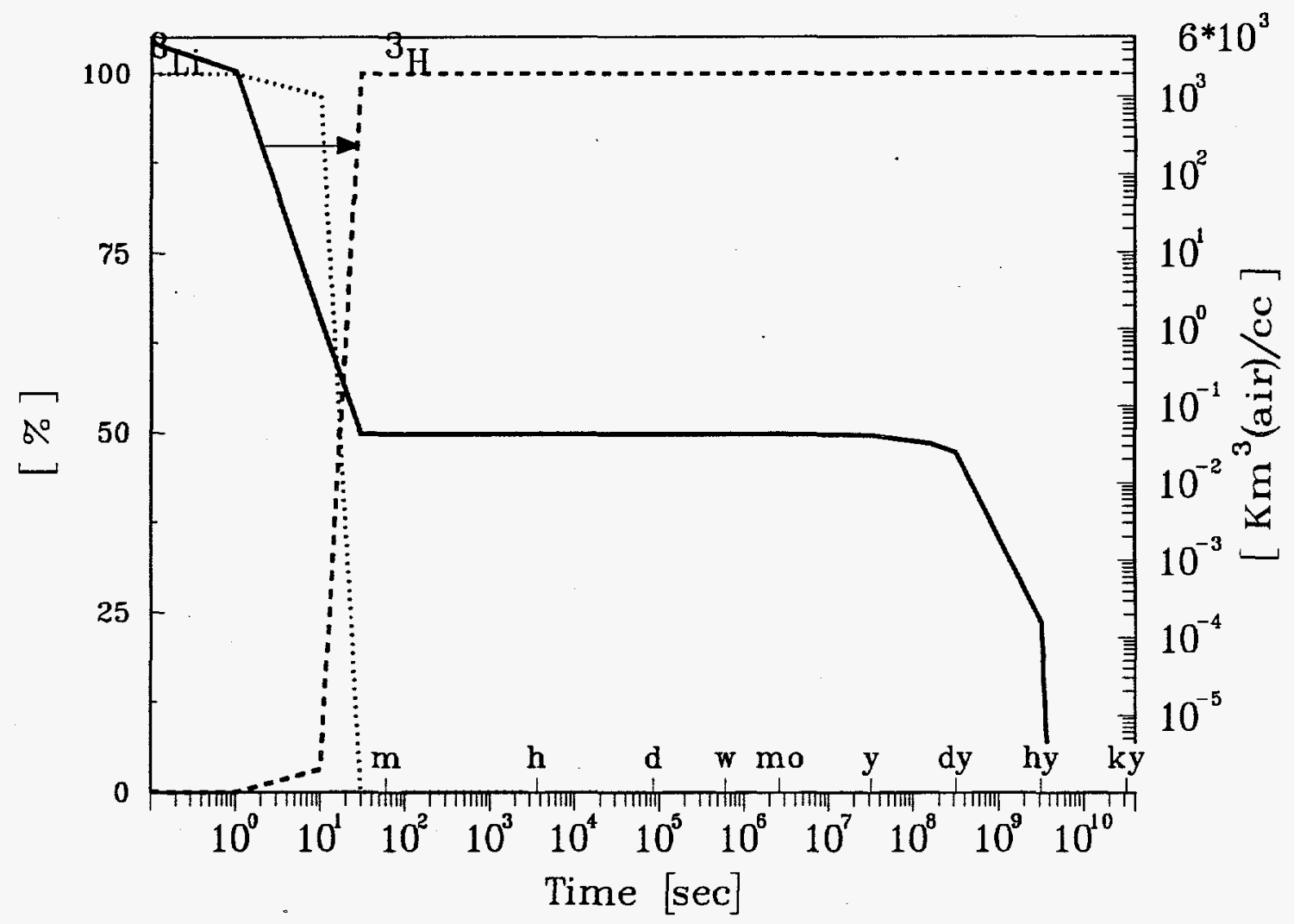

Figure 50. The specific air-BHP in zone \# $18\left(\mathrm{O} \_\mathrm{Li}-\mathrm{Li} / \mathrm{V}\right)$. 

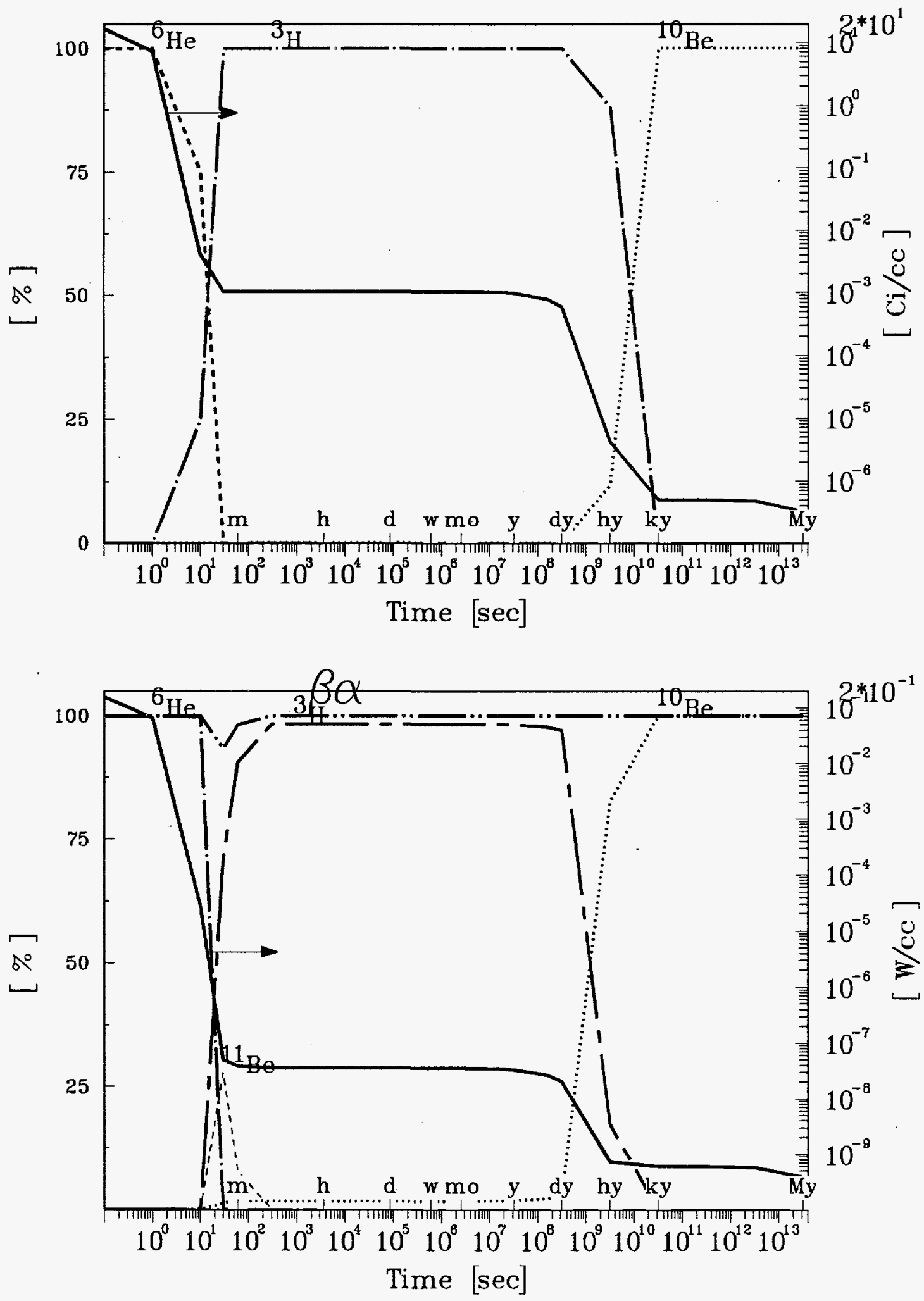

19

Figure 51. The specific radioactivity (top) and the specific decay heat (bottom) in zone \# $19($ O_BeZ - Li/V). 

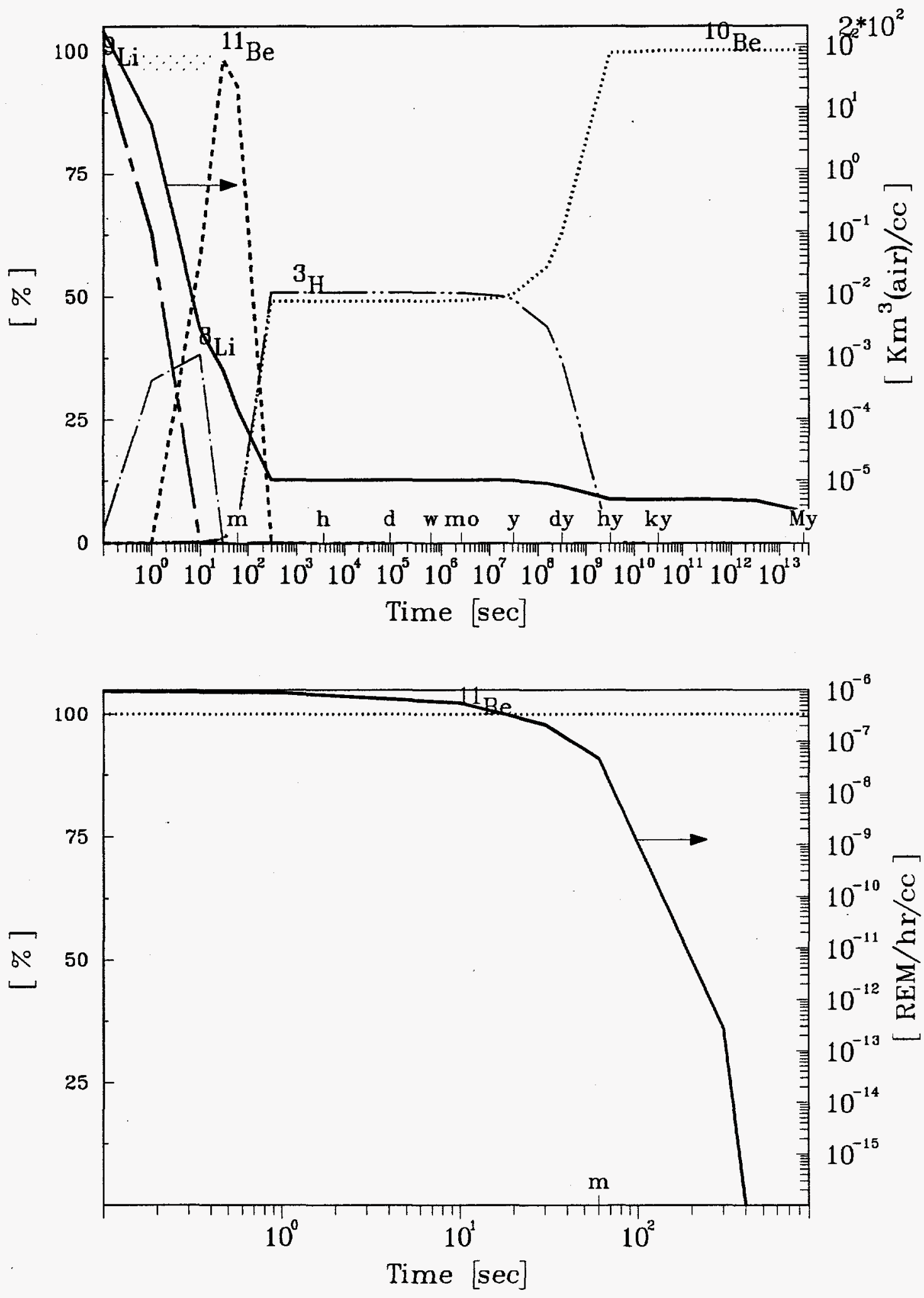

Figure 52. The specific air-BHP (top) and the point-source contact dose (bottom) in zone \# 19 (O_BeZ - Li/V). 

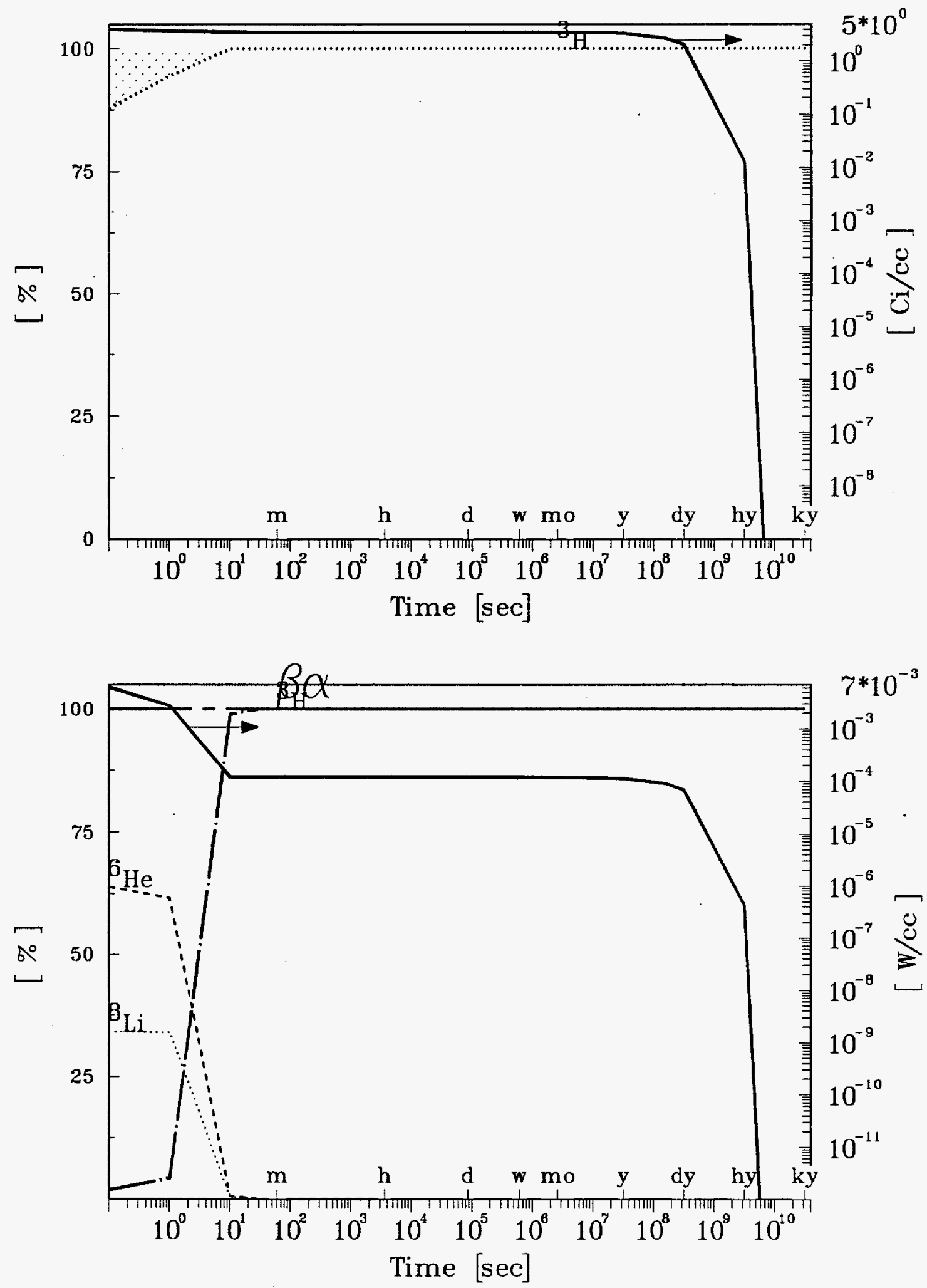

Figure 53. The specific radioactivity (top) and the specific decay heat (bottom) in zone \# $20\left(\mathrm{O} \_\mathrm{Li}-\mathrm{Li} / \mathrm{V}\right)$. 


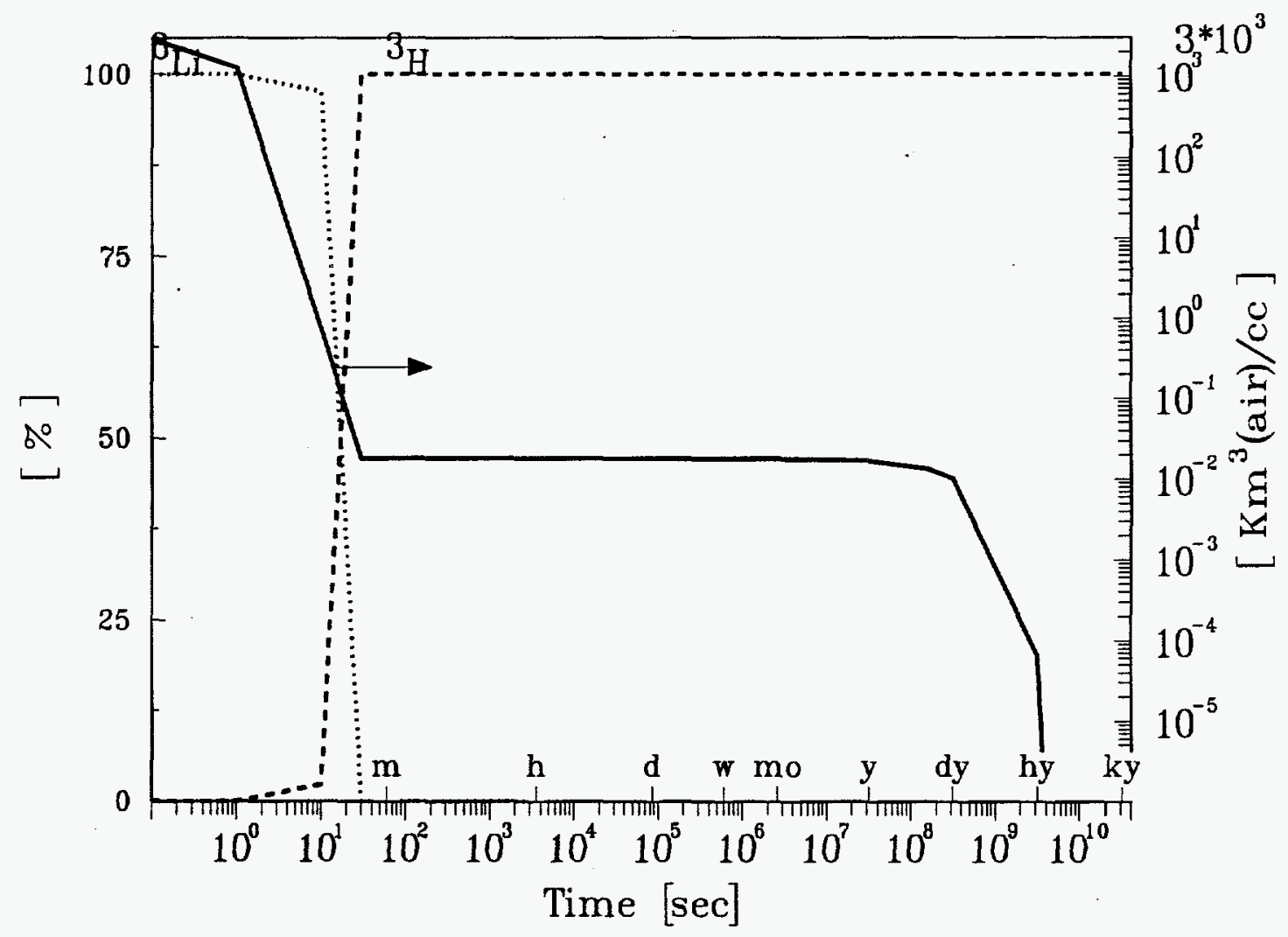

Figure 54. The specific air-BHP in zone \#20 $\left(\mathrm{O}_{-} \mathrm{Li}-\mathrm{Li} / \mathrm{V}\right)$. 

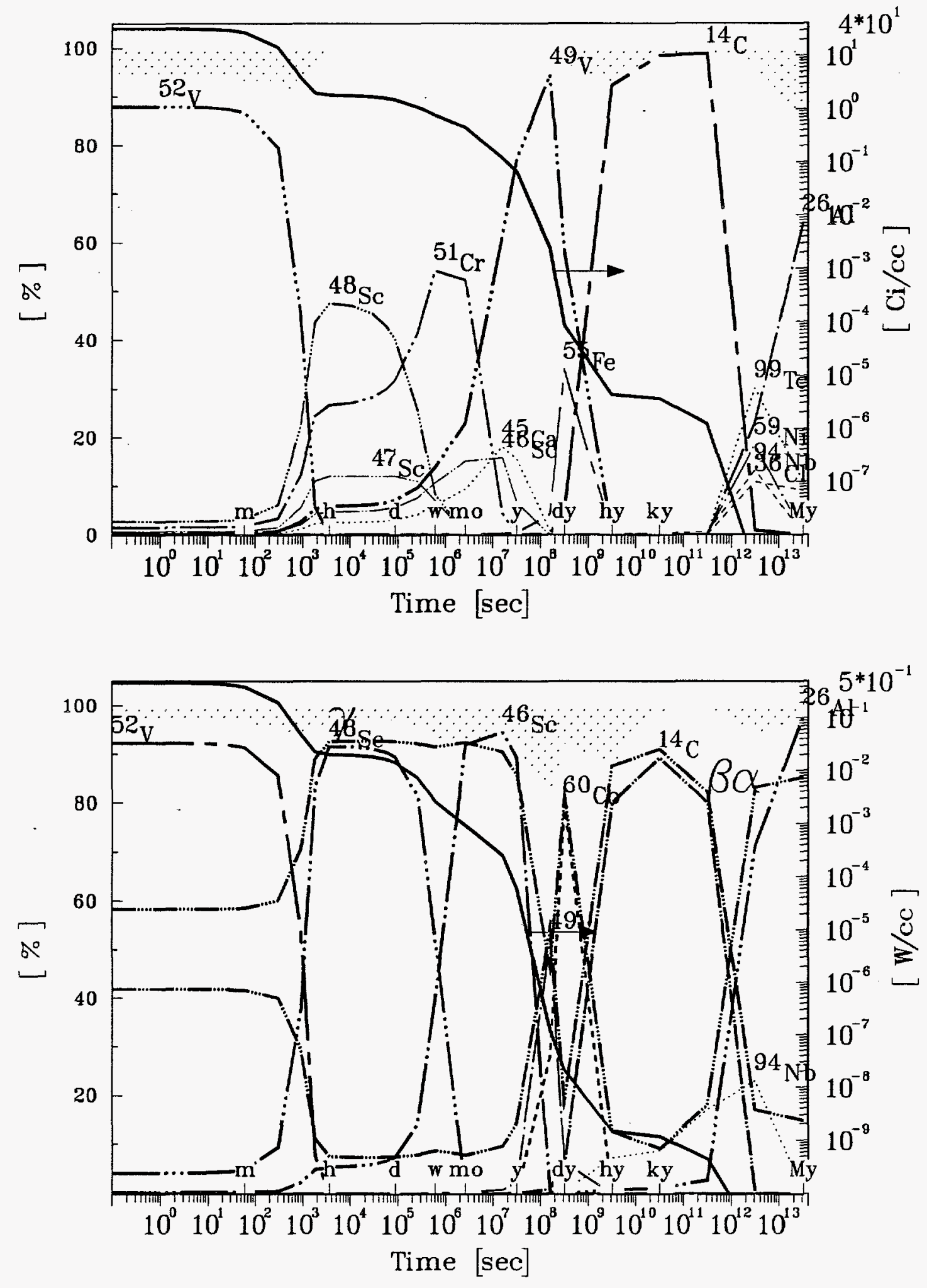

Figure 55. The specific radioactivity (top) and the specific decay heat (bottom) in zone \# 21 (O_Vall - Li/V). 

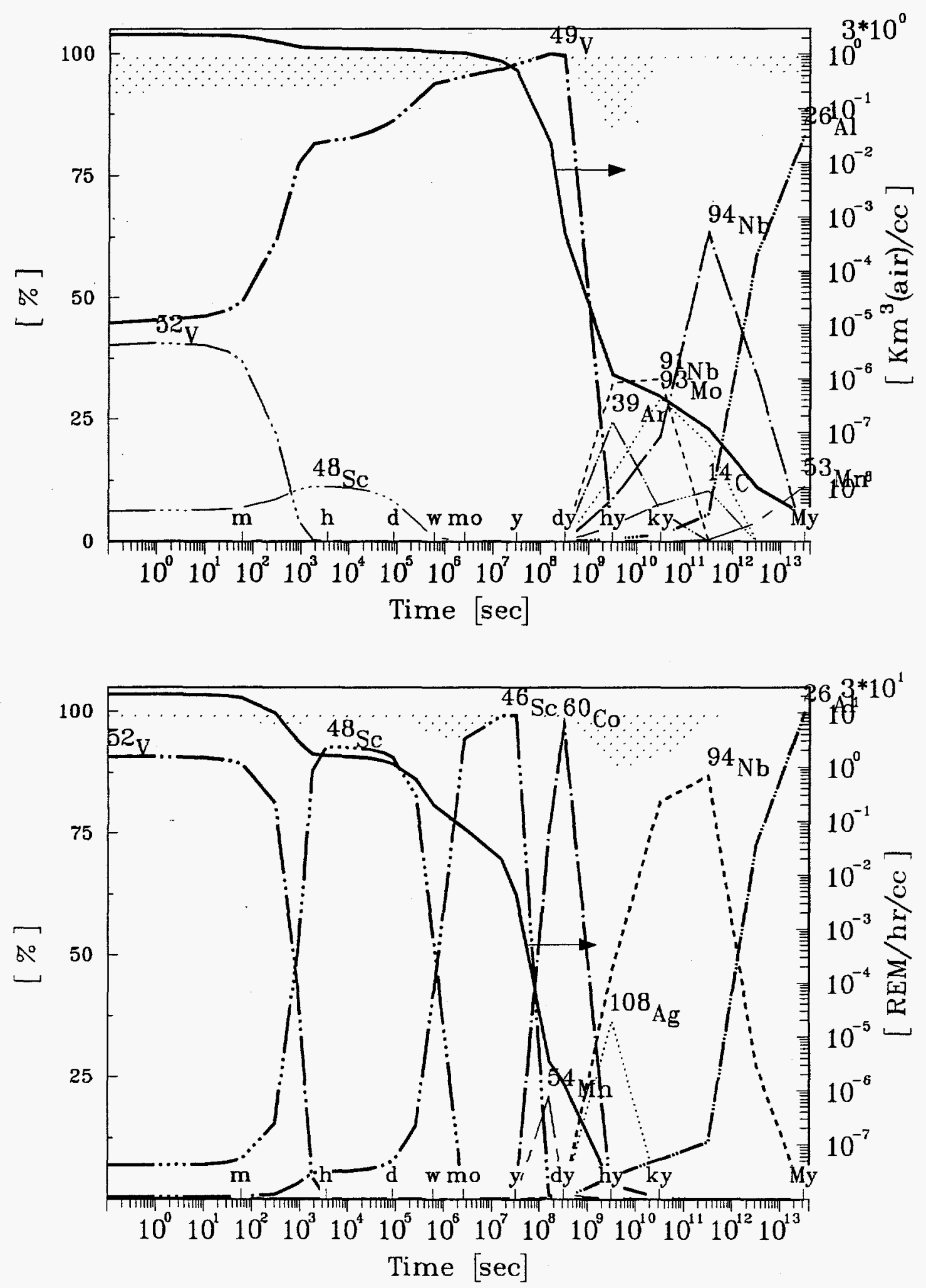

21

Figure 56. The specific air-BHP (top) and the point-source contact dose (bottom) in zone \# 21 (O_Vall - Li/V). 

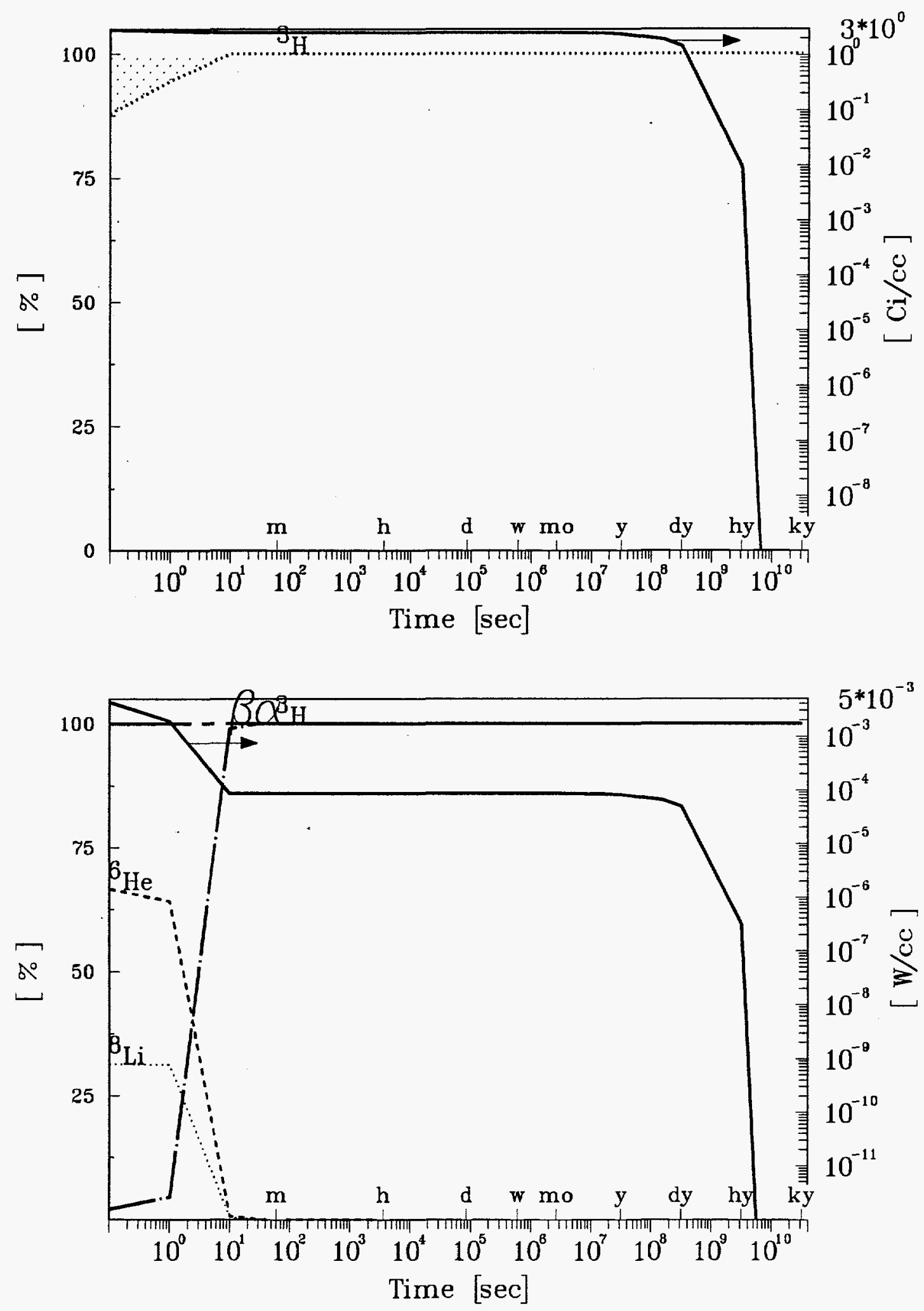

Figure 57. The specific radioactivity (top) and the specific decay heat (bottom) in zone \# $22\left(\mathrm{O}_{-} \mathrm{Li}\right.$ - Li/V). 


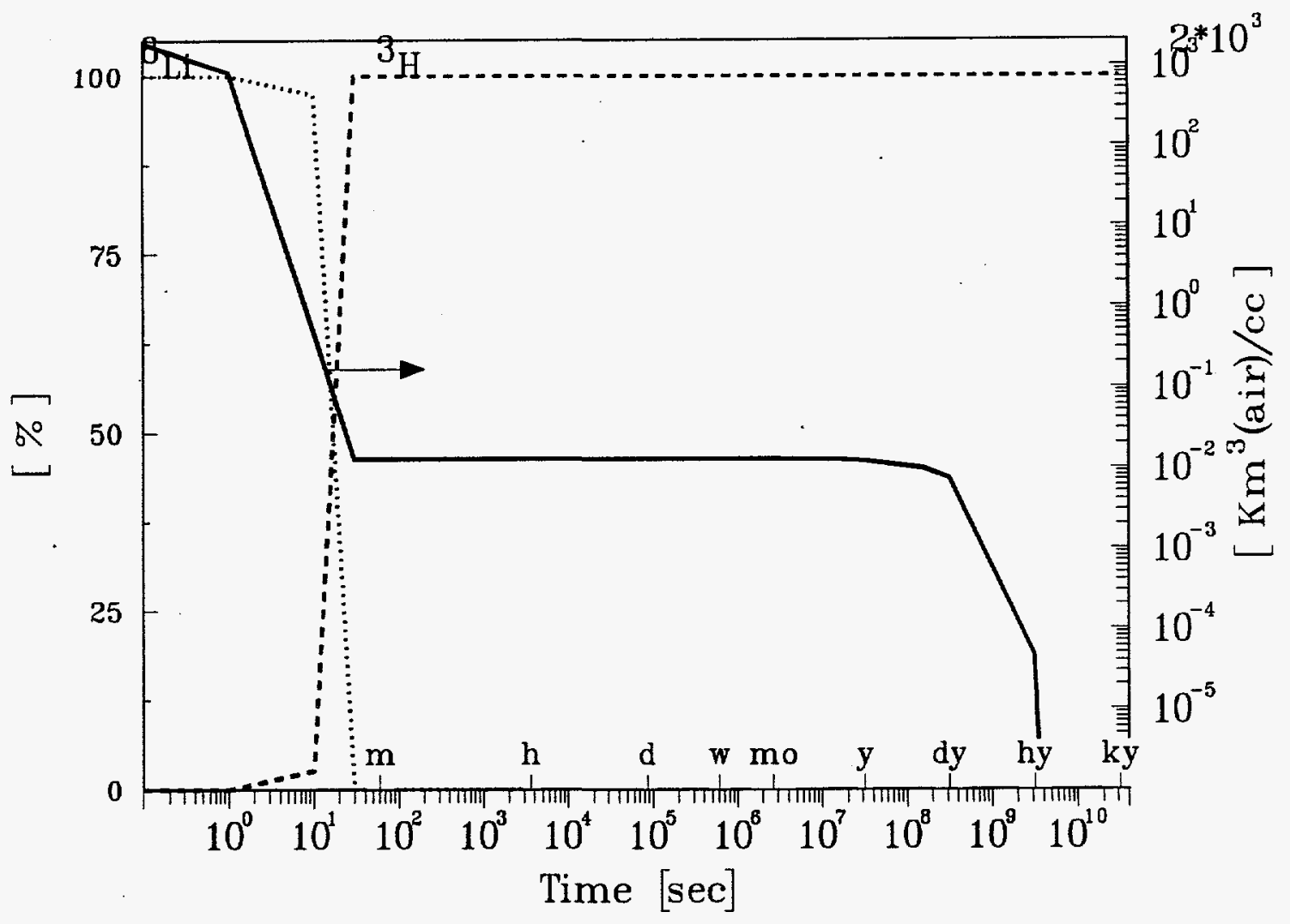

Figure 58. The specific air-BHP in zone \# $22\left(\mathrm{O} \_\mathrm{Li}-\mathrm{Li} / \mathrm{V}\right)$. 

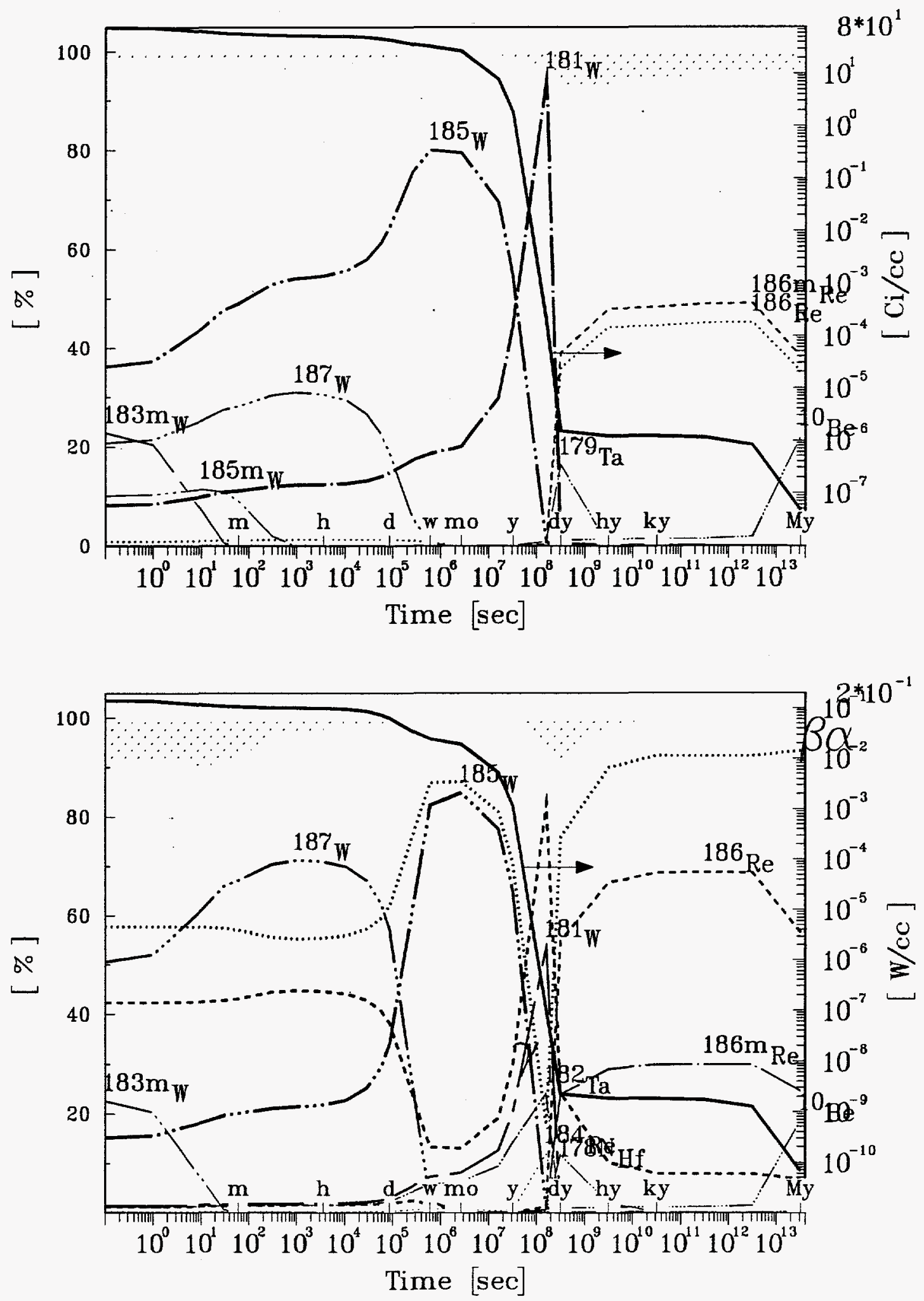

Figure 59. The specific radioactivity (top) and the specific decay heat (bottom) in zone \# 23 (O_WC - Li/V). 

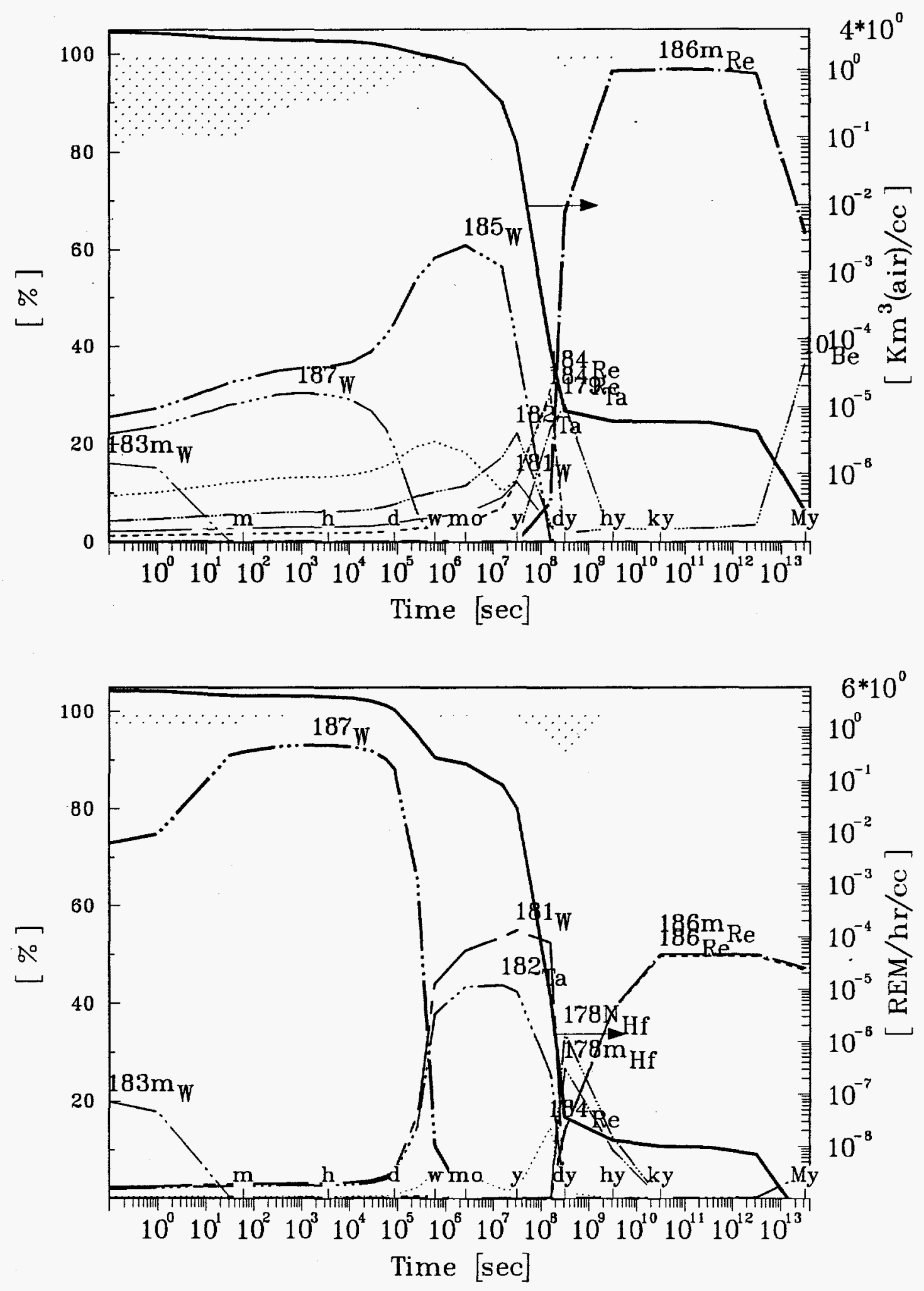

23

Figure 60. The specific air-BHP (top) and the point-source contact dose (bottom) in zone \# 23 (O_WC - Li/V). 

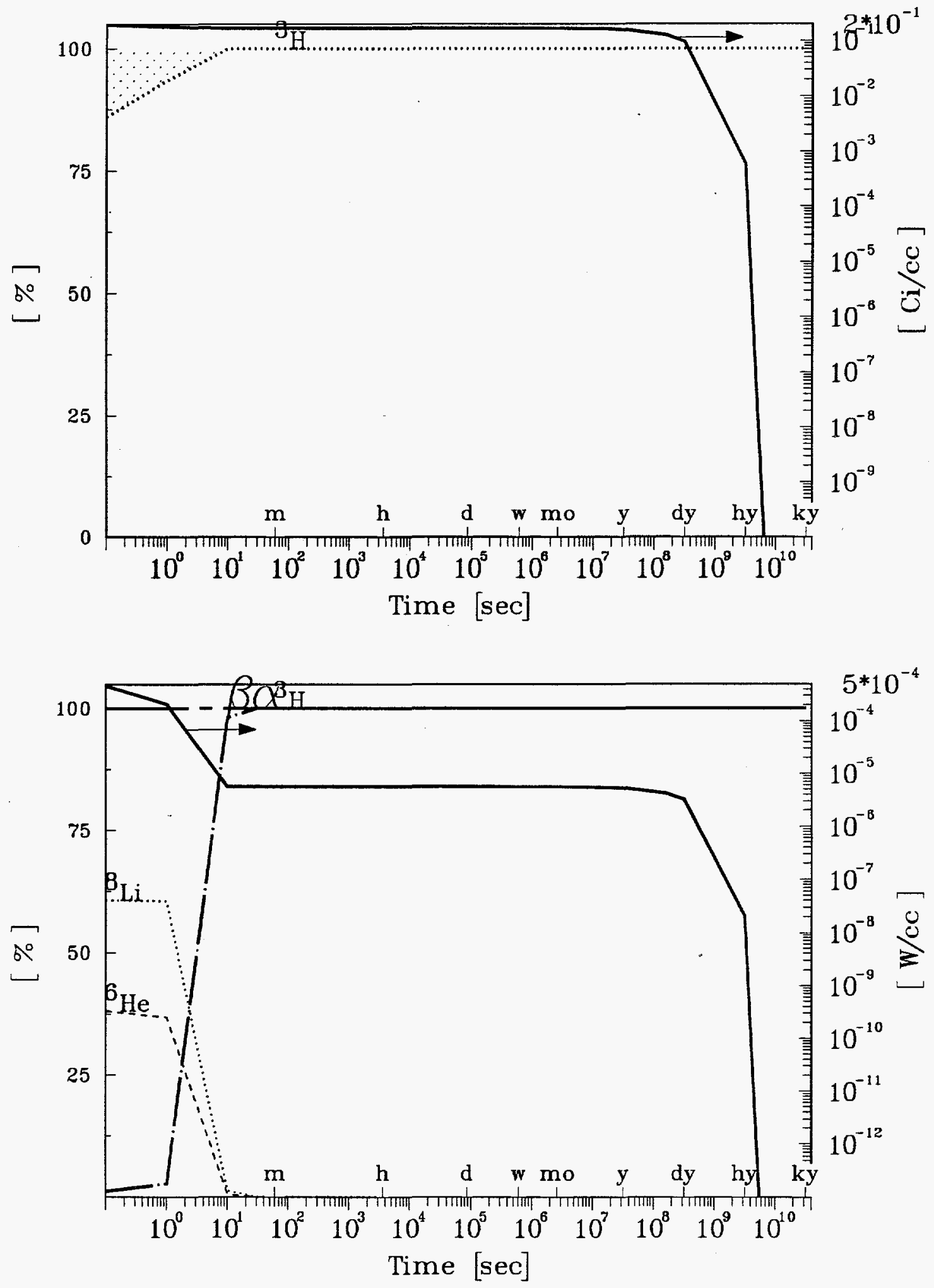

Figure 61. The specific radioactivity (top) and the specific decay heat (bottom) in zone \# 24 (O_Li - Li/V). 


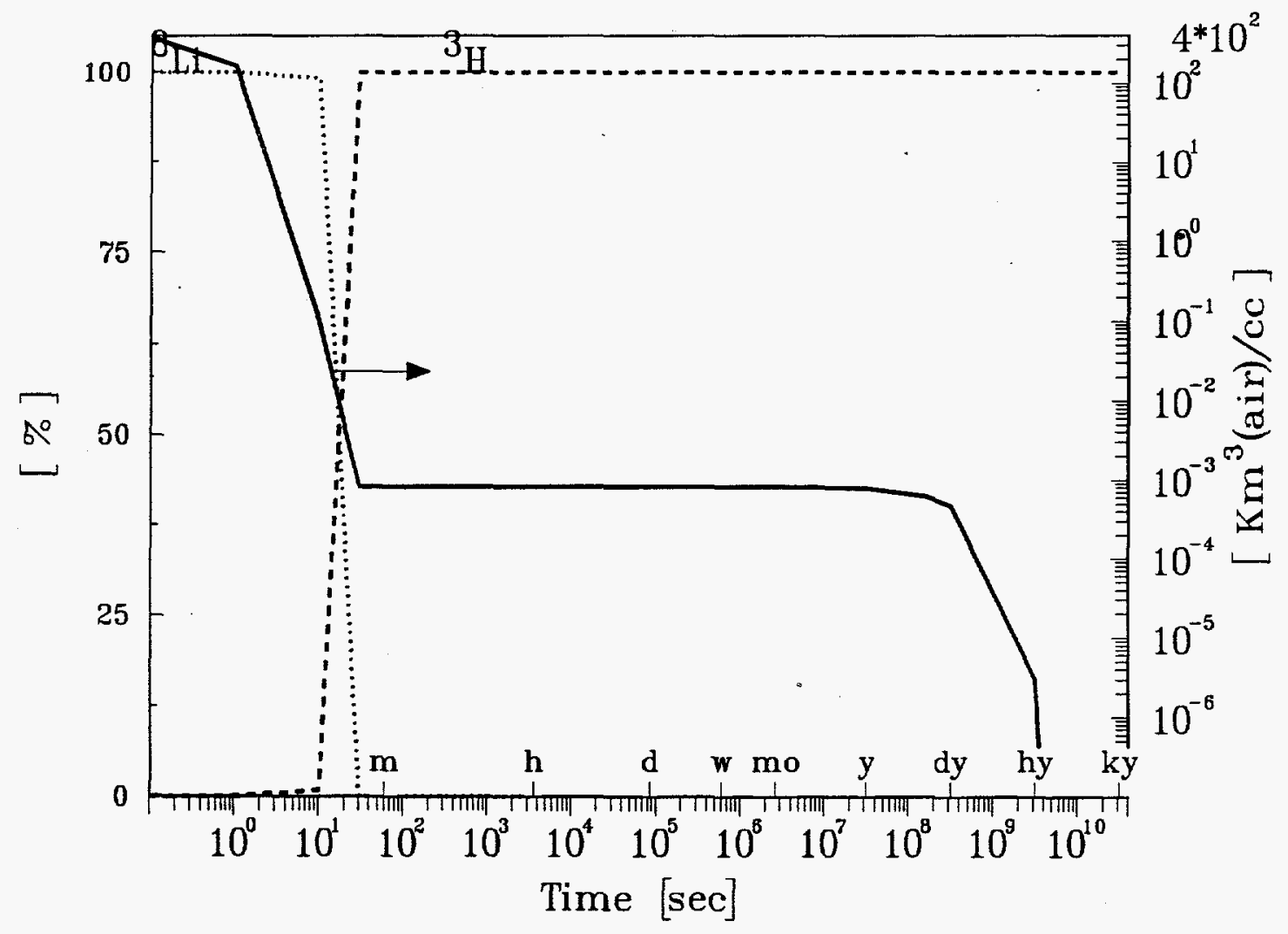

Figure 62. The specific air-BHP in zone \# $24\left(\mathrm{O} \_\mathrm{Li}-\mathrm{Li} / \mathrm{V}\right)$. 

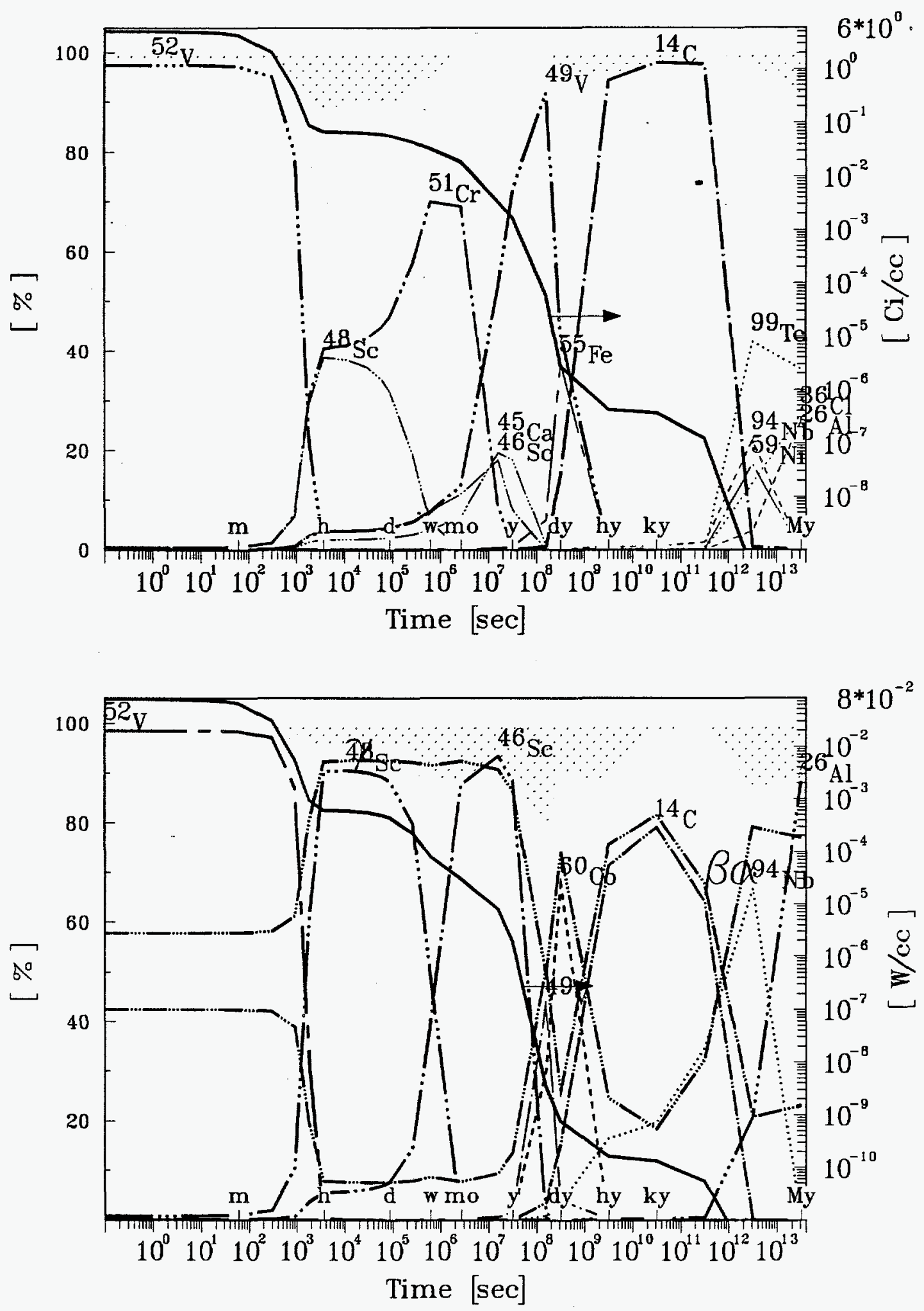

Figure 63. The specific radioactivity (top) and the specific decay heat (bottom) in zone \# 25 (O_Vall - Li/V). 

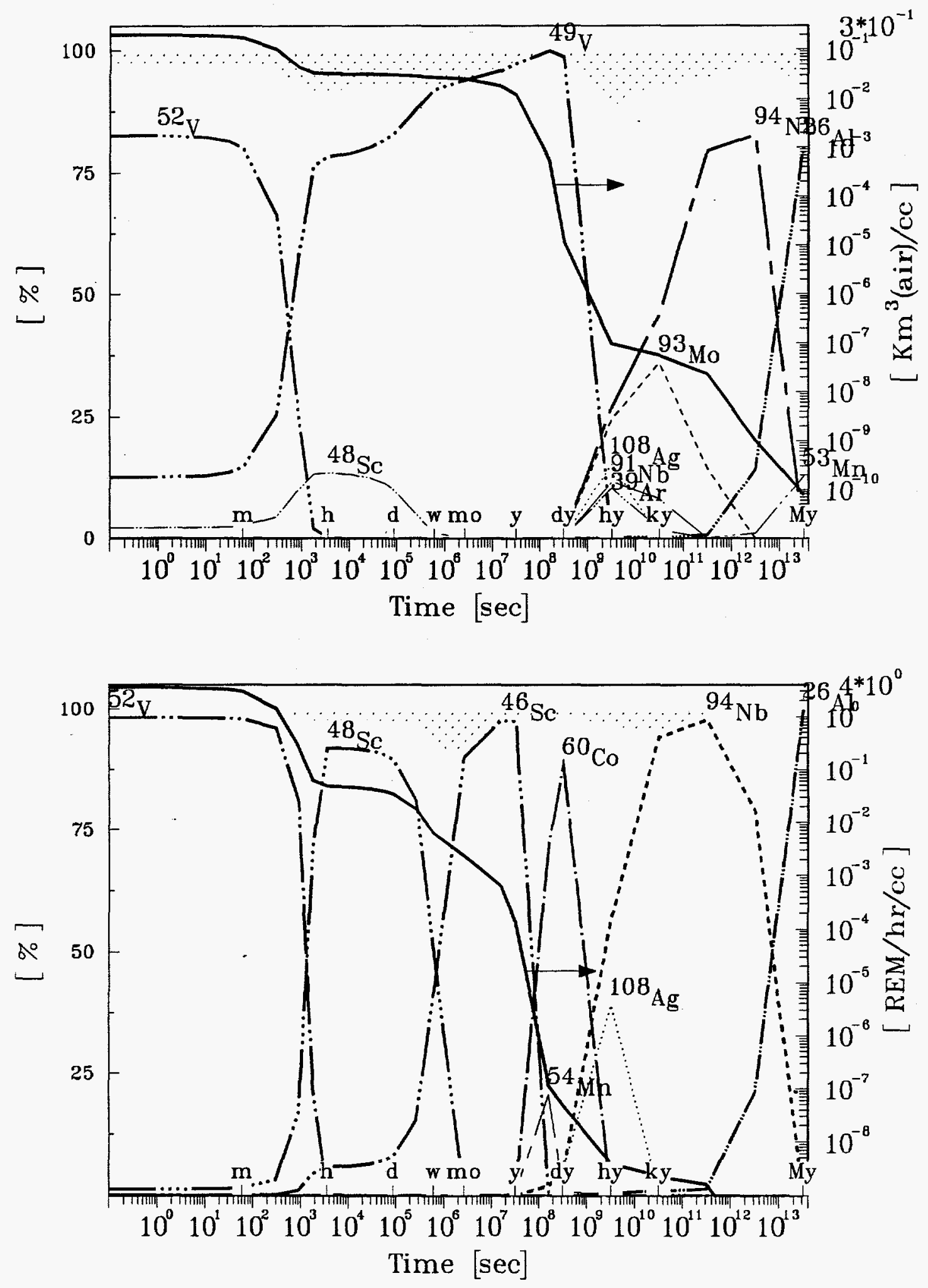

Figure 64. The specific air-BHP (top) and the point-source contact dose (bottom) in zone \# 25 (O_Vall - Li/V). 

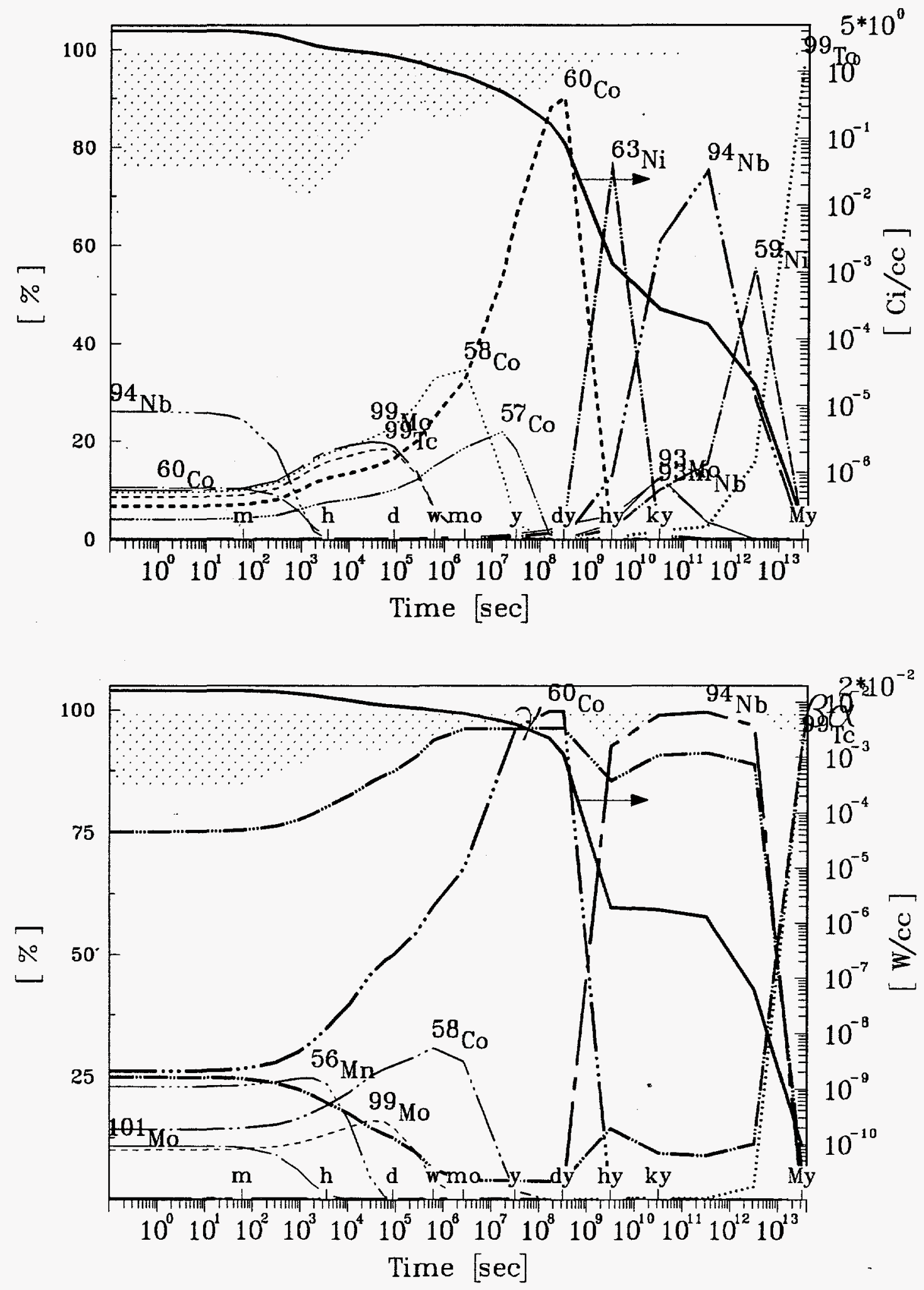

Figure 65. The specific radioactivity (top) and the specific decay heat (bottom) in zone \# 26 (O_Incon - Li/V). 

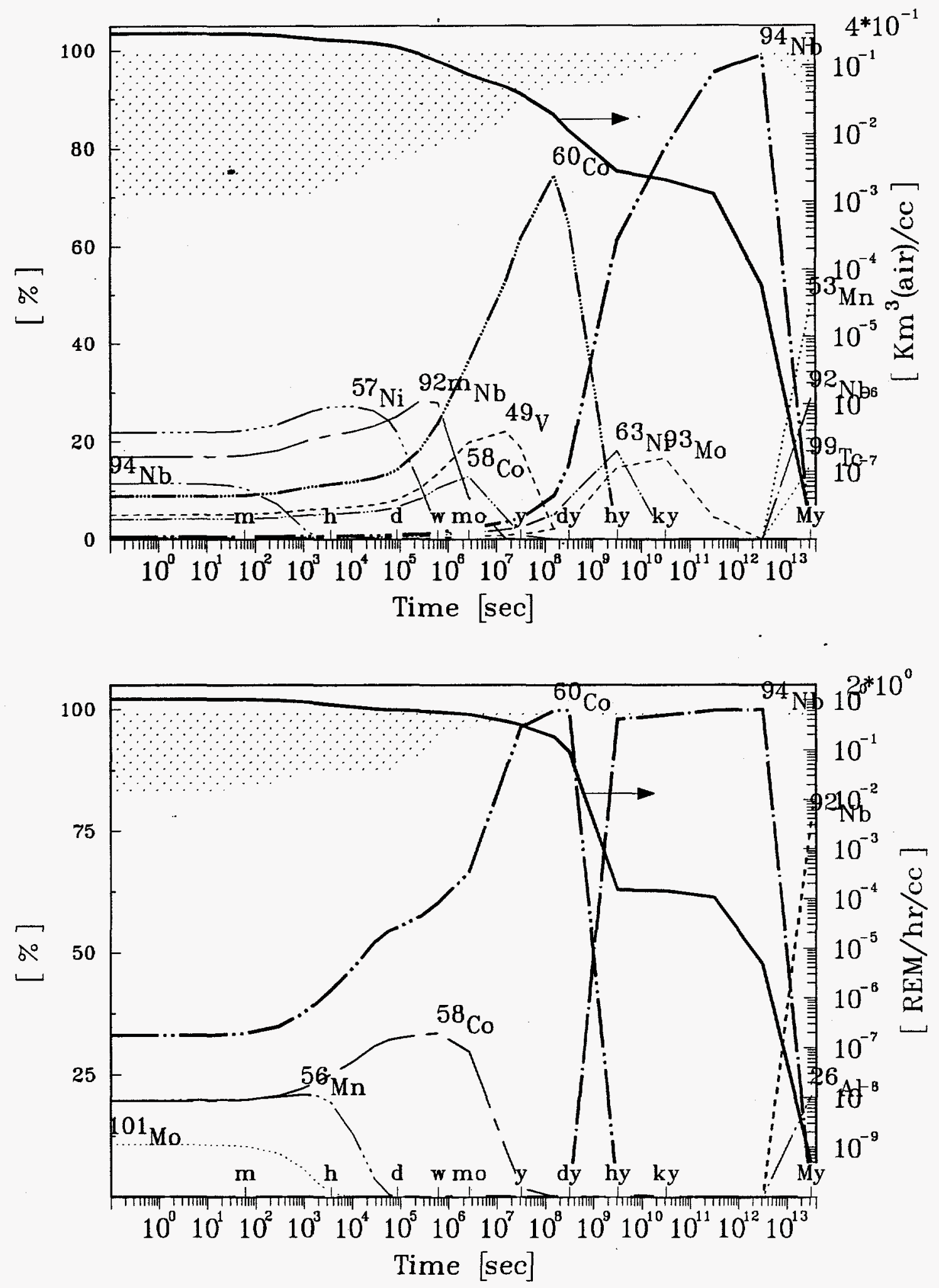

Figure 66. The specific air-BHP (top) and the point-source contact dose (bottom) in zone \# 26 (O_Incon - Li/V). 

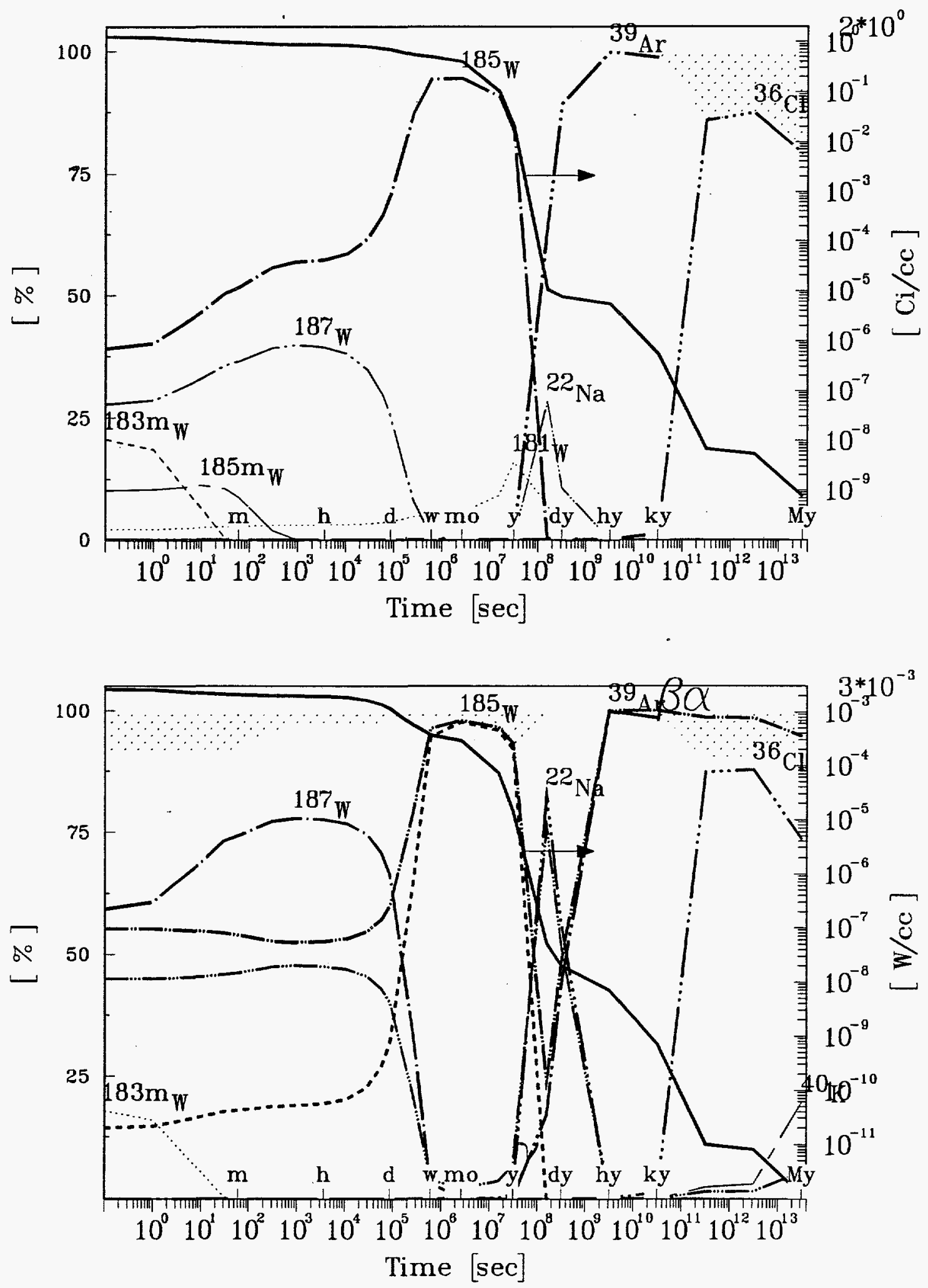

27

Figure 67. The specific radioactivity (top) and the specific decay heat (bottom) in zone \# 27 (O_WNaK - Li/V). 

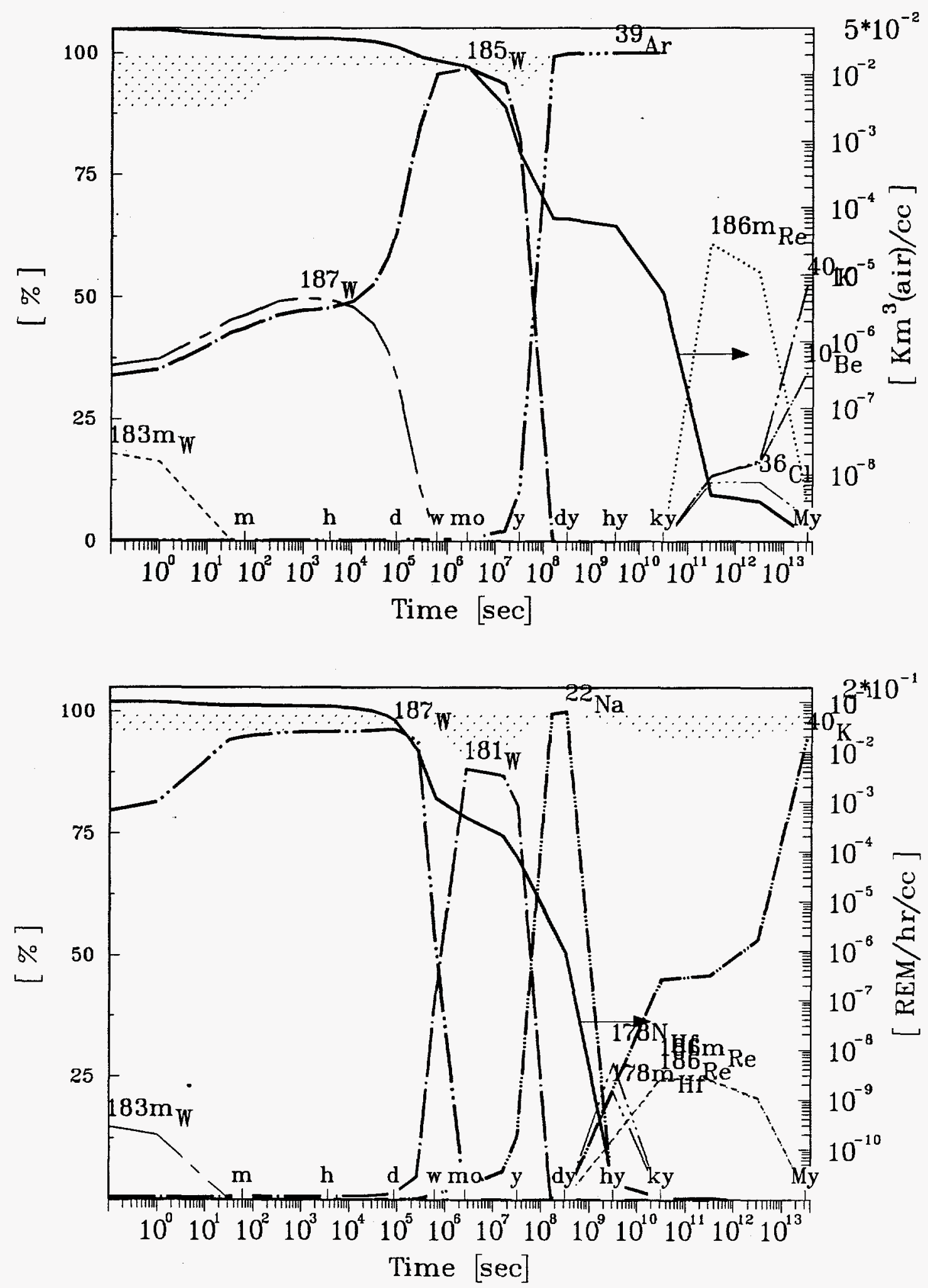

Figure 68. The specific air-BHP (top) and the point-source contact dose (bottom) in zone \# 27 (O_WNaK - Li/V). 

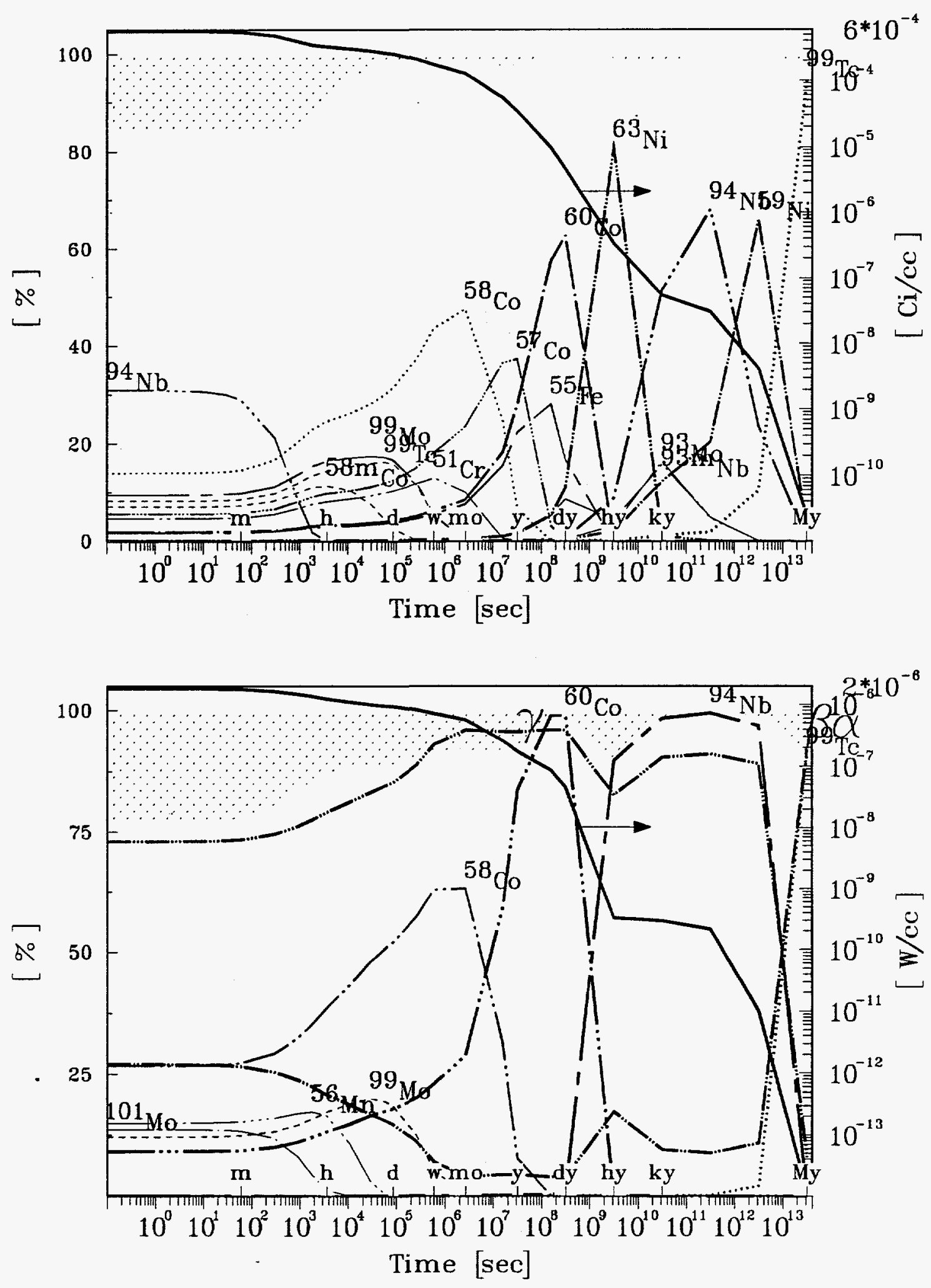

Figure 69. The specific radioactivity (top) and the specific decay heat (bottom) in zone \# 28 (O_Incon - Li/V). 

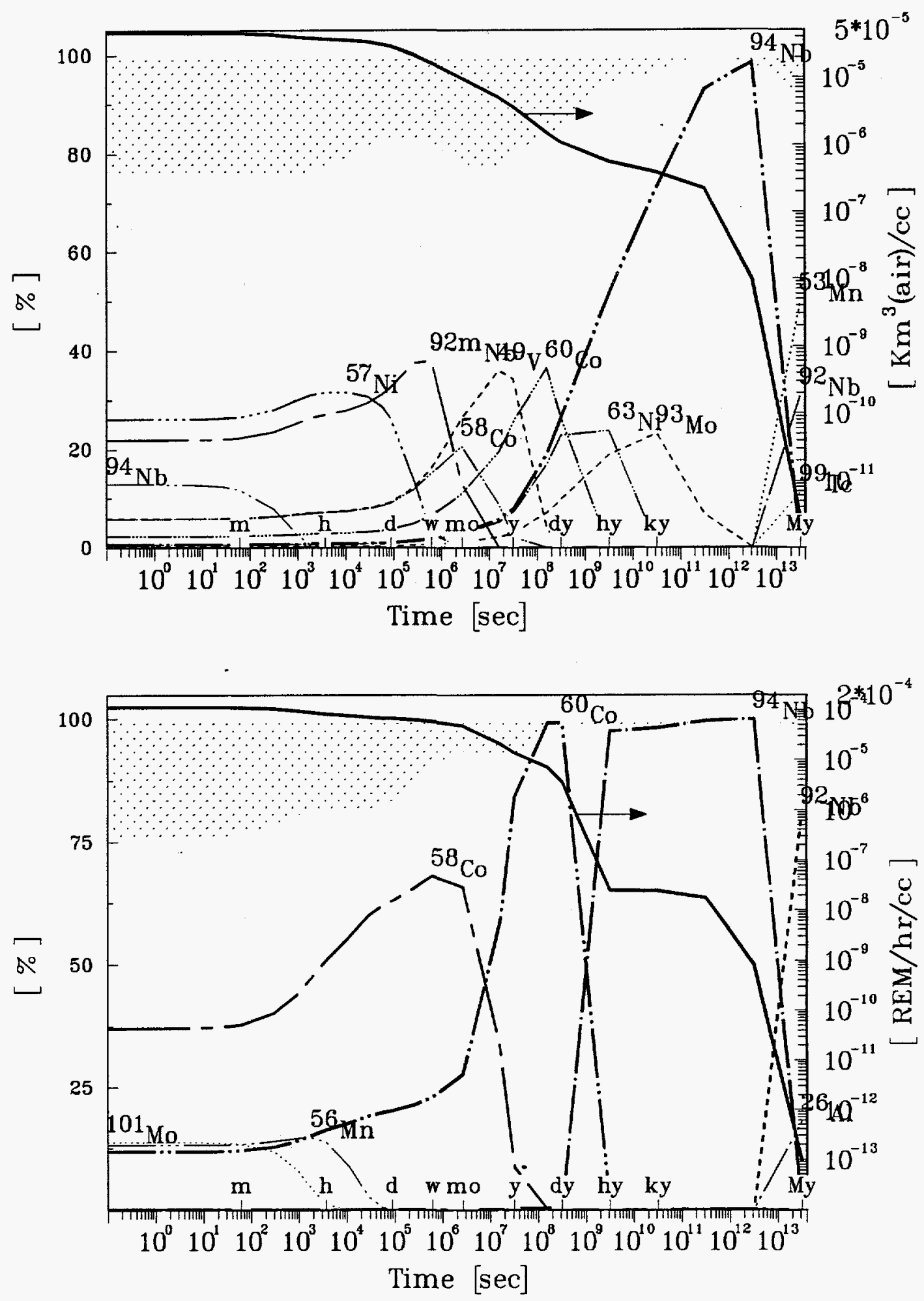

Figure 70. The specific air-BHP (top) and the point-source contact dose (bottom) in zone \# 28 (O_Incon - Li/V). 


\section{References}

[1] H. Attaya, "Input Instructions for RACC-P," Argonne National Laboratory, ANL/FPP/TM-270 (1994).

[2] H. Attaya, "Radioactivity Computation of Steady-State and Pulsed Fusion Reactor Operation," Argonne National Laboratory, ANL/FPP/TM-273 (1995).

[3] R.D. O'Dell et al., "User's Manual for ONEDANT: a code package for onedimensional, diffusion-accelerated neutral particle transport," Los Alamos National Laboratory Report, LA-9184-M (1989).

[4] H. Attaya et al., "Two Dimensional LOCA Analysis for Fusion Reactors," Proc. of 13th IEEE Symposium on Fusion Engineering, Knoxville, TN, IEEE Cat. No. $\underline{89 \mathrm{CH} 2820}, 1120$ (1989).

[5] H. Attaya, "LOCA Analysis for Manganese-Stabilized Steel," Fusion Technology Vol. 19, 1331 (1991). 


\section{DISTRIBUTION LIST FOR ANL/FPP/TM-282}

\section{Internal}
H. Attaya (5)
S. Bhattacharyya
M. Billone
J. Brooks
D. Ehst
Y. Gohar
I. Gomes

A. Hassanein

T. Hua

C. Johnson

M. Lineberry

R. Mattas

B. Micklich

Dale L. Smith

D.-K. Sze

T. Yule

FPP Files (5)

TIS Files (1)

\section{External}

DOE/OSTI for distribution per UC-424 (41)

Manager, Chicago Operations Office

ANL-E Libraries (2)

ANL-W Library

M. Abdou, University of California, Los Angeles

C. Baker, University of California, San Diego

S. Berk, U.S. Department of Energy

W. Daenner, ITER, Germany

J. Davis, McDonnell Douglas Aerospace

J. Doggett, Lawrence Livermore National Laboratory

L. El-Guebaly, University of Wisconsin

U. Fischer, Forschungszentrum Karlsruhe, Germany

W. Gauster, ITER JCT, Garching, Germany

L. Greenwood, Battelle Pacific Northwest Laboratory

I. Kirillov, D.V. Efremov Scientific Research Institute, Russia

D. Lousteau, ITER JCT, Garching, Germany

H. Maekawa, Japan Atomic Energy Research Institute, Japan

S. Malang, Forschungszentrum Karlsruhe, Germany

D. Muntz, Forschungszentrum Karlsruhe, Germany

R. Parker, ITER JCT, Garching, Germany

S. Piet, ITER JCT, San Diego, California

R. Price, U.S. Department of Energy

A. Raffray, ITER JCT, Garching, Germany

G. Saji, ITER JCT, San Diego, California

M. Sawan, University of Wisconsin

M. Seki, Japan Atomic Energy Research Institute, Japan

W. Stacey, Georgia Institute of Technology

D. Steiner, Rensselaer Polytechnic Institute

I. Sviatoslavsky, University of Wisconsin

M. Tillack, University of California, San Diego

J. Vetter, Forschungszentrum Karlsruhe, Germany

G. Vieider, ITER, Germany

R. Watson, Sandia National Laboratories, Albuquerque 
F.W. Wiffen, U.S. Department of Energy

K. Wilson, Sandia National Laboratories, Livermore

M. Youssef, University of California, Los Angeles

C.E.A. Library, Fontenay-aux-Roses, France

Librarian, Culham Laboratory, England

Thermonuclear Library, Japan Atomic Energy Research Institute, Japan 